\title{
The role of $T c$-foxQ2 in the central brain development in Tribolium castaneum
}

\author{
Dissertation \\ for the award of the degree \\ "Doctor rerum naturalium" \\ of the Georg-August-Universität Göttingen \\ within the doctoral program biology \\ of the Georg-August University School of Science (GAUSS) \\ submitted by \\ Bicheng He \\ from Gansu, China
}

Göttingen, 2018 


\section{Thesis Committee}

Prof. Dr. Gregor Bucher (advisor)

Dept. of Evolutionary Developmental Genetics, Johann-Friedrich-Blumenbach-Institute of Zoology and Anthropology, Georg-August-University Göttingen

Prof. Dr. Ernst A. Wimmer

Dept. of Developmental Biology, Johann-Friedrich-Blumenbach-Institute of Zoology and Anthropology, Georg-August-University Göttingen

\section{Members of the Examination Board}

First reviewer: Prof. Dr. Gregor Bucher

Dept. of Evolutionary Developmental Genetics, Johann-Friedrich-Blumenbach-Institute of Zoology and Anthropology,

Georg-August-University Göttingen

Second reviewer: Prof. Dr. Ernst A. Wimmer

Dept. of Developmental Biology, Johann-Friedrich-Blumenbach-Institute of Zoology and Anthropology, Georg-August-University Göttingen

\section{Further members of the Examination Board}

Prof. Dr. Ralf Heinrich

Dept. of Cellular Neurobiology, Schwann-Schleiden Research Center,

Georg-August-University Göttingen

Prof. Dr. Daniel J. Jackson

Dept. of Geobiology, Courant Research Center, Georg-August-University Göttingen

Prof. Dr. Reinhard Schuh

Dept. of Molecular Developmental Biology, Max-Planck-Institute for Biophysical Chemistry

Dr. Kristine Henningfeld

Dept. of developmental Biochemistry, University Medical Faculty Göttingen 


\section{Declaration}

I hereby declare that the doctoral thesis entitled,

"The role of Tc-foxQ2 in the central brain development in Tribolium castaneum " prepared on my own and with no other sources and aids than quoted.

Göttingen, October $16^{\text {th }}, 2018$

Bicheng $\mathrm{He}$ 
To my Family 


\section{Acknowledgement}

The four years PhD journey is a special experience in my life, with a lot of joy, passion and hardship. I am very grateful to those who have given me great support and company during this long journey.

First, I would like to express my sincere gratitude to my supervisor Prof. Dr. Gregor Bucher for his guidance, encouragement and support during my PhD study. His door has been always open for not only scientific discussions but also life problems. He was always optimistic and passionate. He told me that a good scientist should not only do experiments well, but also be good at communicating and showing the results. I really appreciate his encouraging words when I was upset and unconfident.

I would like to thank Prof. Dr. Ernst A. Wimmer for being a member of my thesis committee and for all the suggestions and support.

I would like to express my sincere thanks to Dr. Marita Büscher for her guidance and support. Whenever I had questions and needed help, she was always there for me. She knows a lot about China. I really like the gifts she prepares for me every Chinese new year during my PhD study.

I would like to thank all the members of lab 3 for creating a wonderful working environment. My special thanks goes to Hassan Mutasim Mohammed Ahmed, Dominik Mühlen, Max Farnworth, Natalia Garcia, Yonggang Hu, Salim Ansari, Felix Kaufholz, Vera Terblanche, Peter Kitzmann, Elke Küster, Xuebin Wan, Musa Dan'azumi Isah, Bibi Atika, Constanza Tapia Contreras, Kolja Eckermann, Elisa Buchberger and many others who were always supportive whenever I needed help.

I would like to thank Dr. Frederic Strobl for his effort and patience into this work. I was impressed by his passion for science.

Many thanks go to secretaries, Inga Schild, Birgit Rossi, Constanze Gerhards and Merle Eggers for all the friendly assistance with the bureaucracy stuff. I'm grateful to Inga Schild for helping me with all the registration stuff when I just came to Göttingen. I would like to thank Birgit Rossi. She was such a nice lady and was always willing to help whenever I needed. She helped me a lot with my accommodation. I thank Beate Preitz for solving microscope issues. 
I also want to thank my friends Qian Lang, Na Liu, Hong Pan, Yonggui Wang, Long Li, Dade Yu, Yonggang Hu, Kefei Yang for bringing me so much joy during my stay in Göttingen.

I truly appreciate China Scholarship Council (CSC), Georg-August-University School of Science (GAUSS), Göttingen University for the financial support.

Finally, I would like to thank my parents for always believing in me and supporting me for everything. Special thanks go to my husband, Fan Zhou, who has been always there for me and shared my frustrations and complains throughout my PhD study. I thank him for his great support and any distraction from everyday stress. The four years of PhD, tough but lucky, would be completely different without him. 


\section{Table of contents}

1 Summary

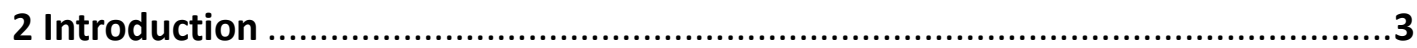

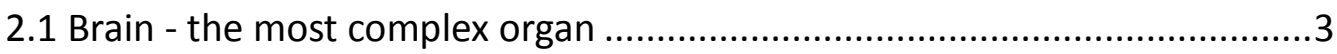

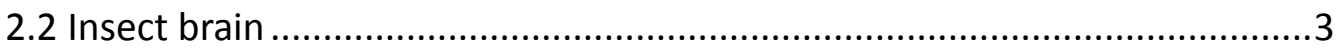

2.3 Early neurogenesis in insect central nervous system ................................

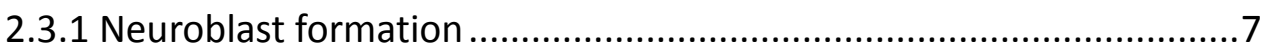

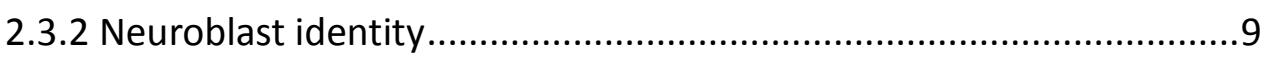

2.3.3 Neuroblast Lineages - type I and type II ........................................10

2.4 Development of insect brain from embryo to adult ................................12

2.5 Development of brain commissures and the central complex ..................13

2.6 Tribolium castaneum - a model organism for the central brain development

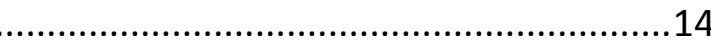

2.7 CRISPR/Cas9 as an approach to generate imaging lines ...........................15

$2.8 T c$-foxQ2 as a candidate gene for the central brain development ..............17

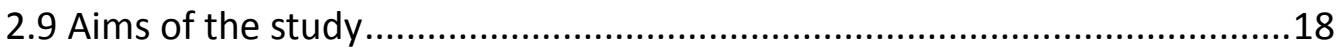

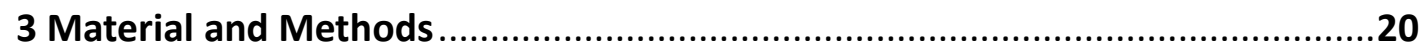

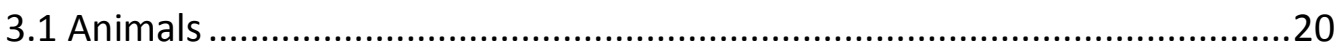

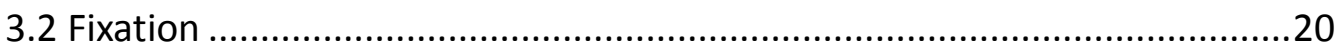

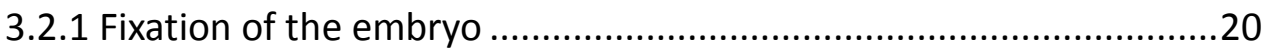

3.2.2 Dissection and fixation of the larval brain.......................................21

3.2.3 Dissection and fixation of the adult brain .....................................21

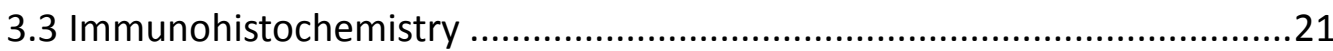

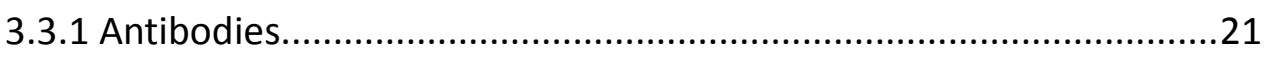

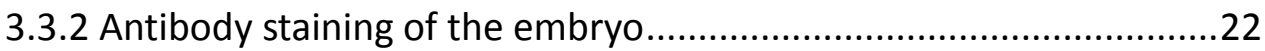

3.3.3 Antibody staining of the larval brain ..............................................22

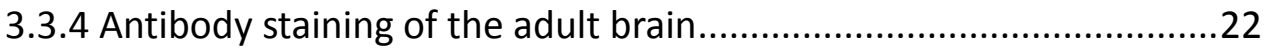

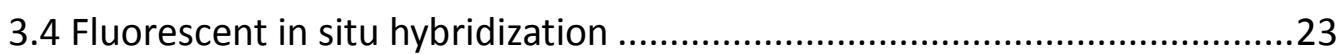

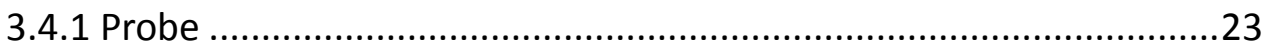

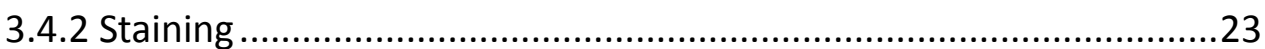

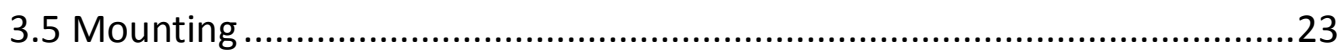

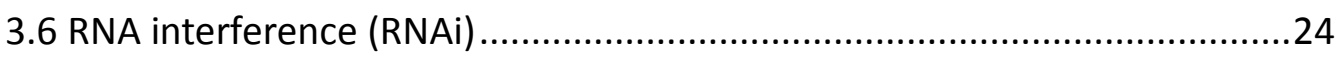

3.7 Generation of a Tc-FoxQ2 polyclonal antibody ........................................24

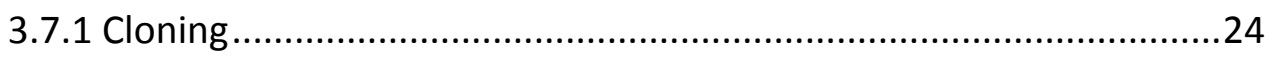

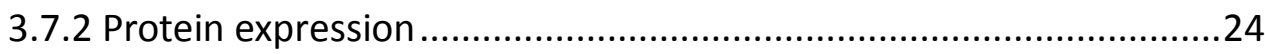

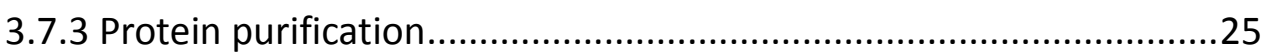

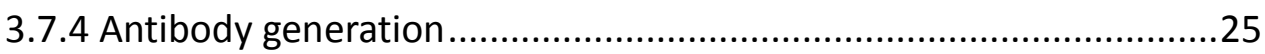

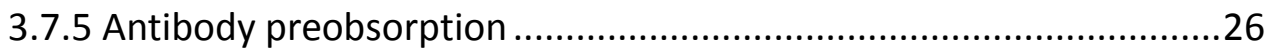

3.8 Generation of a Tc-foxQ2 enhancer trap line by CRISPR/Cas9 ...................26

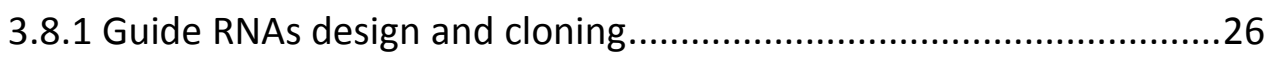

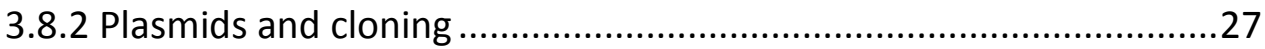

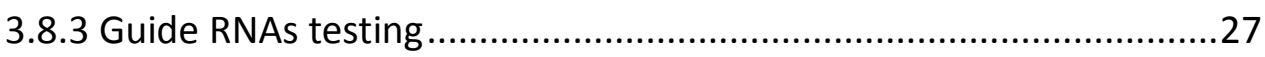


3.8.4 Embryonic injection

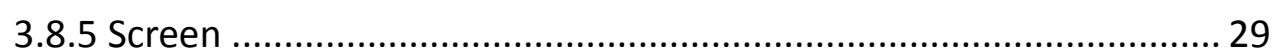

3.8.6 Establish the homozygous stock by genotyping ............................. 29

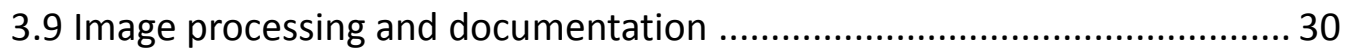

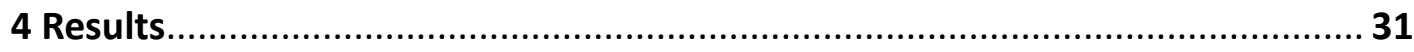

4.1 Generation of a Tc-FoxQ2 polyclonal antibody..................................... 31

4.1.1 Double fluorescent in situ hybridization leads to low resolution at

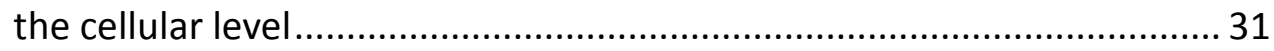

4.1.2 Expression and purification of the Tc-FoxQ2 polypeptide ................ 32

4.1.3 Specificity of the Tc-FoxQ2 antibody ............................................ 34

4.2 Generation of a Tc-foxQ2 enhancer trap line by CRISPR/Cas9 .................. 36

4.3 Generation of Tc-FoxQ2 ${ }^{+}$neuroblasts during embryogenesis ................... 43

4.3.1 Morphological overview of brain development in the embryo ....... 43

4.3.2 Identification of Tc-FoxQ2 ${ }^{+}$neuroblasts in the embryonic brain ...... 44

4.3.3 Candidate genes for Tc-FoxQ2 ${ }^{+}$neuroblasts identity specification .. 47

4.4 Description of the $T c-f o x Q 2$ enhancer trap line........................................ 53

4.4.1 Co-expression analysis of Tc-FoxQ2 and EGFP in the embryo ..........53

4.4.2 Development and contribution of Tc-FoxQ2 ${ }^{+}$cells to the central brain

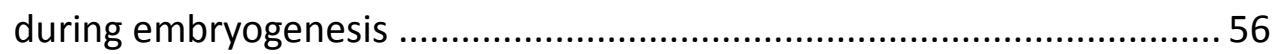

4.4.2.1 Tc-FoxQ2 projects to the contralateral side through the initial axonal commissure in the embryo...................................................56 56

4.4.2.2 FAM2 neuroblast and A-PD neuroblast generate the neural lineages contributing to the central brain primodium ........................ 58

4.4.2.3 Tc-FoxQ2 ${ }^{+}$cells are not glial cells ..........................................60 60

4.4.3 Development and contribution of Tc-FoxQ2 ${ }^{+}$cells to the central brain postembryonically.............................................................................6 60

4.4.3.1 Development and contribution of Tc-FoxQ2 ${ }^{+}$cells in the larval brain 60

4.4.3.2 Development and contribution of Tc-FoxQ2 ${ }^{+}$cells in the adult brain 62

4.5 Tc-foxQ2 Knock-down causes central brain phenotypes in the embryo ....6 64

4.5.1 Tc-foxQ2 RNAi in the Tc-foxQ2 enhancer trap line.........................64

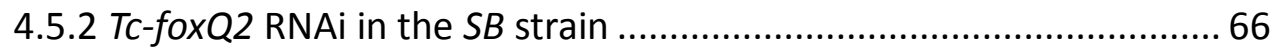

4.5.3 Tc-foxQ2 RNAi in the imaging lines marking the central brain .........68

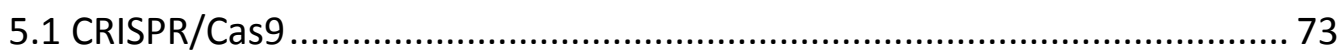

5.1.1 CRISPR/Cas9 mediated knock-in via non-homologous end joining (NHEJ) .73

5.1.2 The survival rate of injected embryos and the concentrations of injection components ......................................................................... 74

5.1.3 The efficiency of CRISPR/Cas9 in Tribolium ................................... 74

5.2 The development of Tc-FoxQ2 ${ }^{+}$cells to the central brain ........................... 76

5.2.1 The enhancer trap line is a good tool to study the development of Tc-FoxQ2 ${ }^{+}$cells 76 
5.2.2 Tc-FoxQ2 lineages contribute to the central complex development.77

5.2.3 Tc-FoxQ2 lineages- type I or type II

5.2.4 Transcription factors are co-expressed in the Tc-FoxQ2 ${ }^{+}$neuroblasts

5.2.4.1 Tc-six3 is expressed in the FAM2 neuroblast and A-PD neuroblast

.80

5.2.4.2 Tc-chx is expressed in the FAM2 neuroblast..........................80

5.2.4.3 Tc-rx and Tc-ey might play a role in specifying the central complex neuroblast

5.3 Tc-foxQ2 is involved in building the axon scaffold in the central brain .......82

5.4 The brain phenotype of Tc-foxQ2 in the embryo ....................................83

5.5 The heterochrony in the central body development .................................84

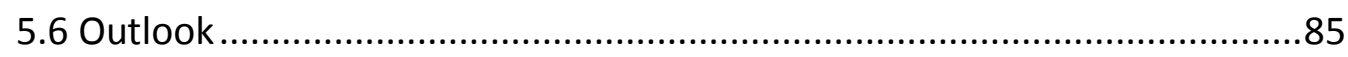

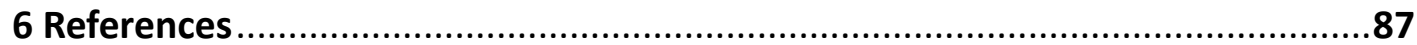

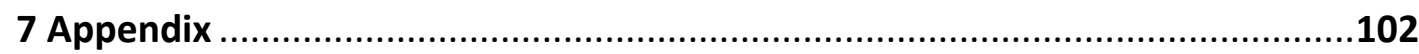

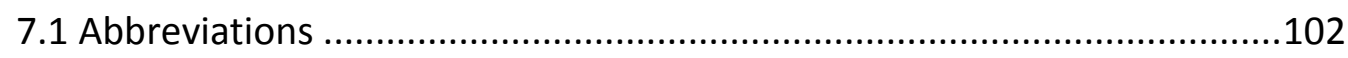

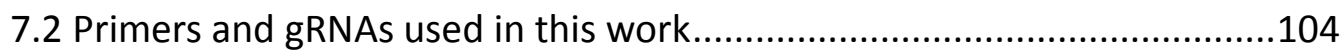

7.3 Quantification of the cell number in this study ....................................106

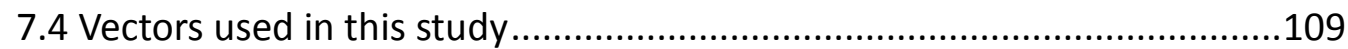

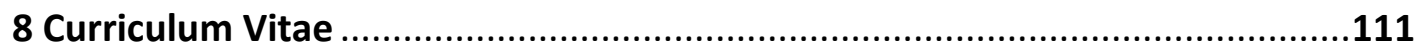




\section{Summary}

The brain is the most complex organ in animals. It controls and coordinates operations like physiology and behavior. The insect brain is built by a conserved set of neuropils which arise from the neuroblasts (NBs). The central complex (CX) is an assembly of neuropils spanning the brain midline, which consists of the protocerebral bridge (PB), central body ( $\mathrm{CB}$ ) with upper and lower unit and the noduli (NO). Drosophila melanogaster and Schistocerca gregaria, are the most studied insect model systems for brain development. Some NBs and lineages contributing to the CX were identified in Drosophila and Schistocerca. However, the molecular and genetic investigations are hampered by the lack of a sequenced genome and the limited potential of the RNAi technique in the latter. Drosophila is not well suited for studying the embryonic signals required for $\mathrm{CX}$ development, since the $C X$ is not detected during the embryogenesis. The red flour beetle Tribolium castaneum, with respect to functional genetics, has more tools than most other insects apart from Drosophila. Most importantly, the CB partially forms during embryogenesis. Tc-foxQ2 has shown to be a key regulatory factor in the anterior median head. It is exclusively expressed in the anterior protocerebral neuroectoderm. Further, $T c$-foxQ2 knock-down was shown to result in central brain phenotypes in Tribolium. However, the embryonic development of the anterior brain is poorly studied. Nothing is known about the function of this gene in Drosophila. In order to get more insights into these processes, I used Tribolium as a model organism to study the role of Tc-foxQ2 in the early brain development, focusing on the $C X$ and central brain development.

In this study, I wanted to study the contribution of $T c$-foxQ2 positive cells to the brain and characterize the function of the $T c-f o x Q 2$ gene. To this end I generated a Tc-FoxQ2 antibody and an imaging line to mark Tc-foxQ2 positive cells by using CRISPR/Cas9 system. With this imaging line, NBs and at least subsets of their lineages contributing to the central brain could be visualized and traced. I identified Tc-FoxQ2 positive NBs at different stages in the embryo and demonstrated that the transcription factors Tc-six3, Tc-six4, Tc-chx, Tc-rx, Tc-ey, $T c$-scro and Tc-fez1 are co-expressed in these Tc-FoxQ2 positive NBs. Further, I was able to show that Tc-FoxQ2 positive cells projected to the contralateral side through the initial axonal commissure of the brain, but did not pioneer the primary brain commissure. Further, I found that the identified FAM2 NB generated a median lineage and the A-PD NB generated a lateral lineage, which projected axon fascicles into the central brain primordium in the late embryo. In the adult brain, 
the median lineage and lateral lineage generated axon bundles projecting into the PB. Moreover, Tc-foxQ2 is required for the formation of midline crossing projections of a number of cell groups. knock-down of Tc-foxQ2 leads to the aberrant formation of the brain commissures at later stage, but did not affect the formation of the initial brain commissures at an earlier stage. Additionally, I found a medial fusion of the brain hemispheres as described before and the reduction of a number of cells which contribute to the central brain. Finally, the reduction of EGFP signal and number of marked cells in Tc-foxQ2 RNAi in the Tc-foxQ2 enhancer trap line indicated a self-regulatory function of this gene. Taken together, this study reveals an important function of Tc-foxQ2 in central brain formation. 


\section{Introduction}

\subsection{Brain - the most complex organ}

The brain is the one of the most important organs in animals, which serves as the most advanced part of the central nervous system. The brain controls and coordinates the complex operations like behavior, memory, movements and orientation. Due to this essential role for survival, it is likely that the morphology and function of the brain undergo high evolutionary pressure. The vertebrate brain is highly complex so that it complicates the study of the brain development. Until recently, it still has difficulties in investigating the cellular and molecular mechanisms of brain development in vertebrates. This is in part due to the billions of neurons in the vertebrate brain (Reichert and Boyan, 1997). A mouse brain contains $\sim 75$ million neurons (Oh et al., 2014). By contrast, the insect brain is smaller and simpler than the vertebrate brain, which consists of dramatically fewer neurons. For example, Drosophila melanogaster has a tiny brain which comprises just 200000 neurons in the adult brain (Younossi-Hartenstein et al., 1996; Urbach and Technau, 2004; Younossi-Hartenstein et al., 2006). Methods are now available to identify the cells and visualize the structures in the insect brain. The molecular techniques and transgenic tools are highly developed in Drosophila, which make it as the preferred model organism for the molecular and genetic investigations of the brain development (Reichert and Boyan, 1997; Urbach and Technau, 2003b; Younossi-Hartenstein et al., 2006). The grasshopper is an ideal model organism for cellular analysis due to the large size of the neuroblasts (NBs) in the brain. It has been shown that the size of the NBs in the grasshopper brain is almost three times larger than in Drosophila (Doe and Goodman 1985). It is known that insects and vertebrates have homologous genes that are conserved in functions (McGinnis and Krumlauf, 1992; Reichert and Boyan, 1997). It is very likely that the analysis of genes which play importance roles for the brain development in insect may lead to the discovery of homologous genes that are equally important in the vertebrate brain. For these reasons, the insect brain has become an important model system to study the developmental processes of the brain.

\subsection{Insect brain}

The insect brain derives from the anterior neuroectoderm which forms the supraesophageal ganglion. This ganglion, also simply termed 'brain', consists of 
three major parts: the protocererbrum (PC), the deuterocerebrum (DC) and the tritocerebrum (TC) (Figure 2.1A, B). The subesophageal ganglion consists of the fused mandibular, maxillary and labial neuromeres, which are structured similarly to those of the segmental ganglia of the VNC that develop from the ventral neuroectoderm (Reichert and Boyan, 1997). The PC is the largest portion of the brain. It includes the intrinsic neuropils: the mushroom bodies (MBs), the central complex (CX), the lateral accessory lobes (LALS) and the optic lobes (OLs). The DC is smaller comprising the antennal lobes (ALs) and the TC is associated with the intercalary segment (Figure 2.1C; Reichert and Boyan, 1997; Kurylas et al., 2008).

The mushroom bodies (MBs) are a pair of protocerebral brain structures comprising Kenyon cells, calyces and lobes. The thousands of Kenyon cells are densely packed and extend dendrites into the calyx. And the axons from Kenyon cells project through the peduncle and form five distinct lobes (the vertical $\alpha$ and $\alpha^{\prime}$ lobes and the medial $\beta, \beta^{\prime}$ and $\gamma$ lobes). The MBs are known to be essential for learning and memory (Armstrong et al., 1998; Kurusu et al., 2002; Strausfeld et al., 2003; Tanaka et al., 2008; Strausfeld et al., 2009). The optic lobes (OLs) which are located in the lateral PC are the visual processing center of the insect brain. In Drosophila, the OLs contain more than $60 \%$ of the brain's neurons and consist of three distinct neuropils: the lamina, medulla and lobula complex (Elphick et al., 1996; Homberg et al., 2011; Nériec and Desplan, 2016). The antennal lobes (ALs) are the glomerular architectures in the deutocerebrum of insects and serve as a primary olfactory center that transmits odor information from the antennae to higher brain centers (Stocker et al., 1990; Marin et al., 2002; Jefferis et al., 2007). The central complex $(C X)$ is a midline spanning neuropil in insect brain. It consists of the protocerebral bridge (PB), the fan-shaped body (FB), the ellipsoid body (EB) and the paired noduli (NO). The FB and EB are also called upper unit and lower unit of the central body (CB). The basic modules of the $C X$ are highly conserved across insect species (Williams 1975; Hanesch et al., 1989; Wegerhoff and Breidbach, 1992; Rein et al., 2002; Loesel et al., 2002; Homberg 2008; El Jundi et al., 2010; Dreyer et al., 2010). The CX is a higher order integration center in the insect brain. It is known to be involved in sky compass orientation, locomotor behavior, courtship, and memory (Strauss 2002; Homberg 2008; Weinrich et al., 2008; Pfeiffer and Homberg 2014). 
A

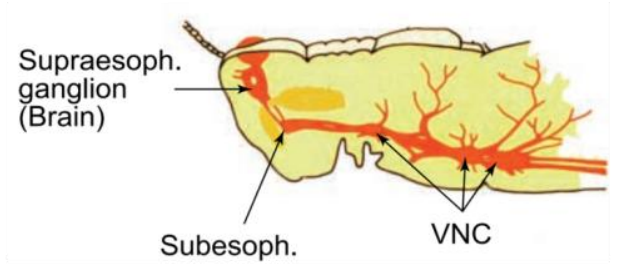

B

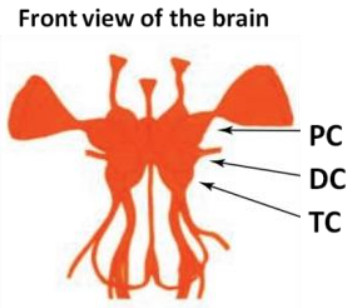

C

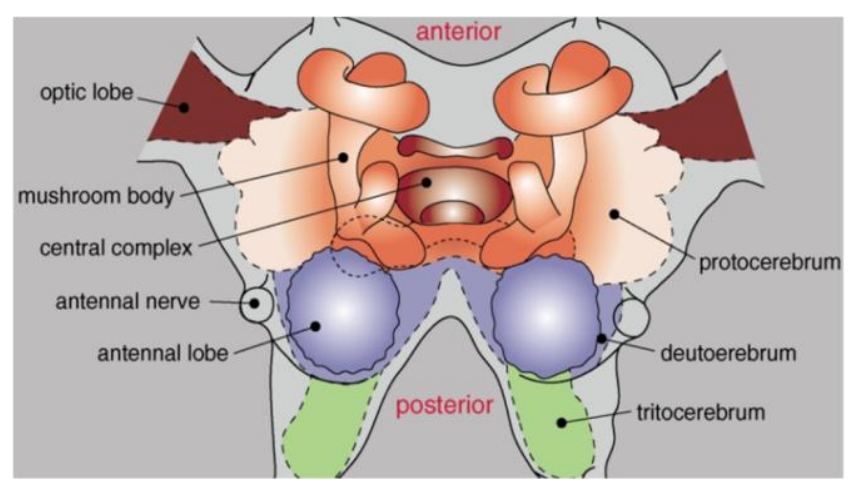

Figure 2.1 Structural organization of the insect brain. (A) Orientation of the brain (or supraoesophageal ganglion) and the subesophageal ganglion within the head capsule of the grasshopper. (B) and (C) Schematic view of the adult brain, exhibiting the protocerebrum (PC), the deutocerebrum (DC), the tritocerebrum (TC) and various neuropils. The PC comprises the mushroom bodies, the central complex, and the optic lobes. The DC encompasses the antennal lobes. (Taken from Reichert and Boyan, 1997; Urbach and Technau, 2003a)

The basic neural architectures in the adult brain in insects are conserved but the morphological diversity reflects the species diversity, such as shape, size and development time of neuropils (Figure 2.2; Figure 2.3). For instance, the MBs of the honey bees show a larger volume as compared to the desert locusts (Brandt et al., 2005; Koniszewski et al., 2016). Compared with the vinegar flies, the red flour beetles have smaller OLs (Dreyer et al., 2010; Koniszewski et al., 2016). On the other hand, the timing of the development of the neuropils also varies in different species. In Drosophila, the first identifiable CX appears in the third instar larva, showing the immature PB and FB (Young and Armstrong 2010). In tenebrionid beetles, such as Tribolium and Tenebrio, a partially formed CB can be detected in the late embryos, which successively develops until the adult stage (Wegerhoff and Breidbach 1992; Wegerhoff et al., 1996; Koniszewski et al., 2016). In grasshopper, the CB develops fully during embryogenesis (Boyan and Williams 1997, 2011; Williams et al., 2005; Boyan and Reichert, 2011). 
(a)

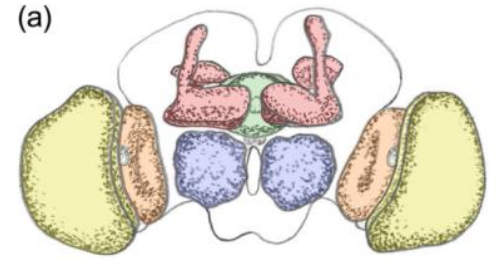

Vinegar fly Drosophila melanogaster

(c)

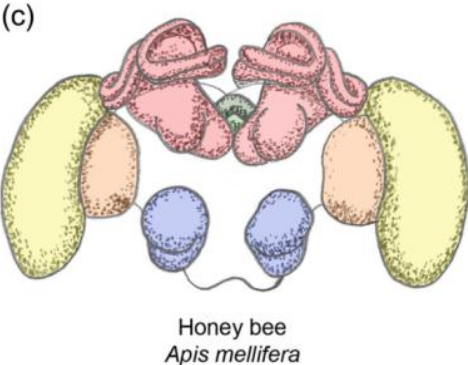

(b)

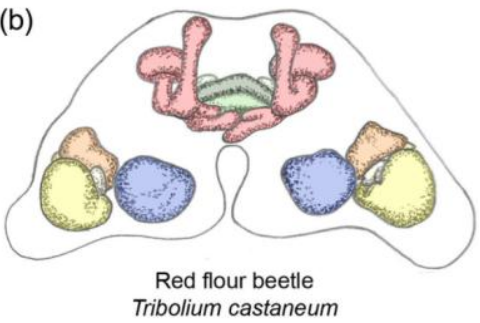

(d)

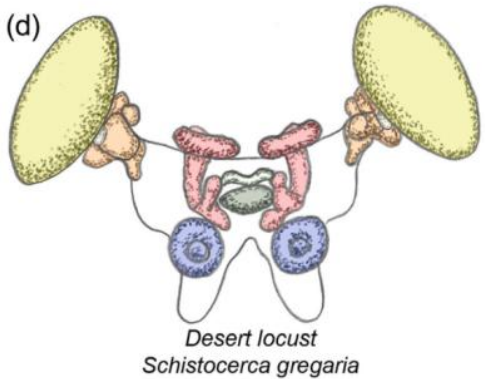

Figure 2.2 The diversity of the adult brain in different species. (a) Vinegar fly Drosophila melanogaster, (b) red flour beetle Tribolium castaneum, (c) honey bee Apis melifera and (d) desert locus Schistocerca gregaria. The respective neuropils are shown in the same color: mushroom bodies (MBs, red), central complex (CX, green), antennal lobes (ALs, blue) and optic lobes (OLs, yellow and orange). Lamina of optic lobes (yellow) and lobula of optic lobes (orange). The figure is taken from (Koniszewski et al., 2016) based on (Rein et al., 2002; Kurylas et al., 2008; Dreyer et al., 2010; Rybak et al., 2010).

(a)

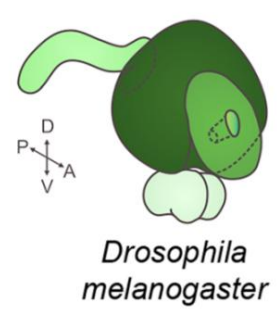

embryonic

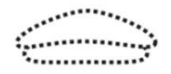

postembryonic

adult (b)
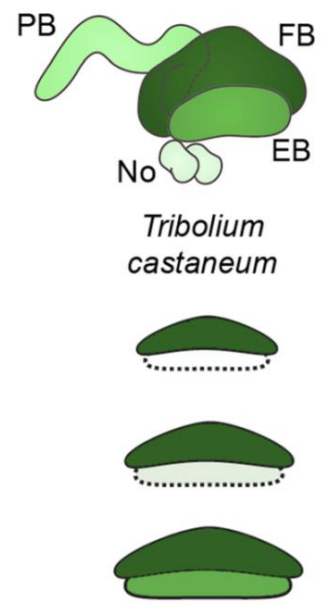

(c)
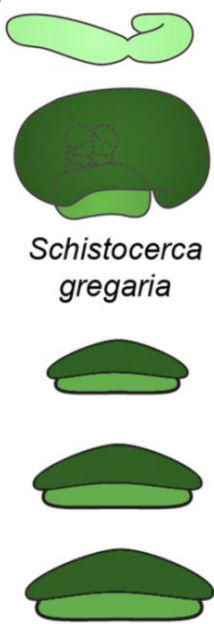

Figure 2.3 The timing of the CX development of Drosophila melanogaster (a), Tribolium castaneum (b) and Schistocerca gregaria (c). In Drosophila CB is absent during embryonic stages, in Tribolium only the FB is developed and in Schistocerca the CB is fully developed. Light colors mark developing but not functional neuropils while white demonstrates the absence of detectable neuropil. PB protocerebral bridge; No noduli; FB fan-shaped body; EB ellipsoid body. The figure is taken from (Koniszewski et al., 2016) based on (Dreyer et al., 2010; Hanesch et al., 1989; Kaiser, 2014). 


\subsection{Early neurogenesis in insect central nervous}

\section{system}

\subsubsection{Neuroblast formation}

The neurogenesis begins with the delamination of the neuroblasts (NBs) from the neuroectoderm. The neuroectoderm (also referred to as neurogenic region) gives rise to the brain and the ventral nerve cord (VNC), which comprise the central nervous system (CNS) of insects. The equipotent cells that are clustered in groups at invariant positions in the neuroectoderm, called proneural clusters, express the proneural genes achaete, scute and lethal of scute which are combined in the achaete/scute gene complex (AS-C) at the beginning of neurogenesis (Campuzano et al., 1985; Cabrera et al., 1987; Skeath et al., 1992). The expression of the proneural genes confers neural potential to all cells within such a proneural cluster. However, only one cell of the proneural cluster adopts the neural fate to become the NB, while the remaining others adopt the epidermal fate to differentiate into the progenitors of epidermis (epidermoblasts) (Figure 2.4A). This selection is regulated by Notch signaling pathway through the mechanism of lateral inhibition (Figure 2.4B; Cabrera et al., 1987; Skeath and Carroll 1992; Heitzler et al., 1996; Beatus and Lendahl, 1998). High levels of Notch signaling inhibit the expression of its ligand Delta and the proneural genes in the surrounding cells. Therefore lateral cells adopt the epidermal fate. Meanwhile, the selected proneural cell, which continues to expresses high levels of the proneural gene and Delta, acquires the NB fate. 


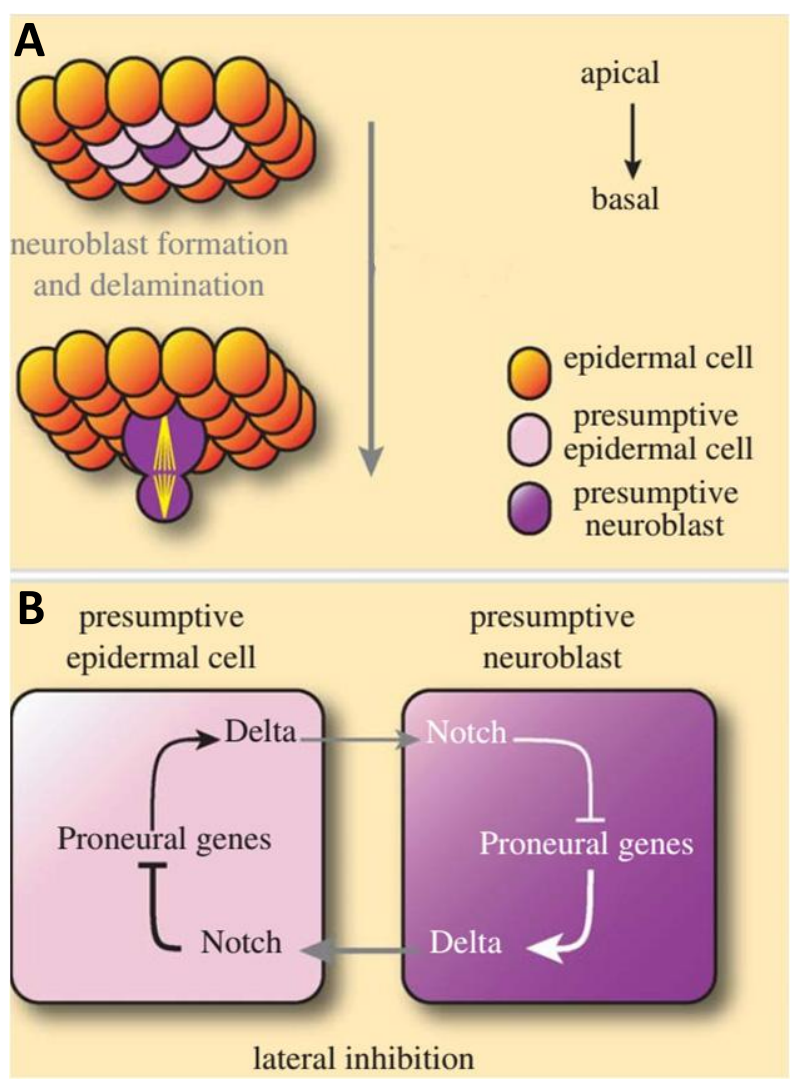

Figure 2.4 Schematic drawing of NBs formation and the lateral inhibition involving Notch, Delta and the proneural genes. (A) A single cell is selected to acquire a NB fate from the proneural cluster and the remaining cells adopt an epidermal fate. This is achieved by the process of lateral inhibition (B). (Taken from Egger et al., 2008)

In Drosophila, $230 \mathrm{NBs}$ delaminate from the ventral neuroectoderm in each hemisegment within five temporal waves and form a stereotypical array. These NBs are assigned in seven anteroposterior rows and three dorsoventral columns. These 30 NBs totally generate approximately 350 progeny which build a hemineuromere (Schmidt et al., 1997). The fate of the individual NB is specified by its position, time of formation as well as the combination of developmental genes it expresses (Doe 1992; Urbach and Technau, 2004; Hartenstein et al., 2008). Compared to the VNC, the brain shows a much higher complexity. The formation of NBs in the brain seems not to follow the apparent row-column patterning. This is mainly owing to massive morphogenetic movements during development and an expansion of number of NBs forming the brain. In Drosophila, 100 NBs have been identified in the embryonic brain. They form in a reproducible time pattern rather than in waves. Their spatial arrangement is largely invariantly. Each NB is generated at a characteristic time (Urbach and Technau, 2004). The protocerebral neuroectoderm gives rise to $\sim 70 \mathrm{NBs}$, taking the largest portion of NBs population in the brain. The deutocerebrum is formed from $\sim 21 \mathrm{NBs}$ and the tritocerebrum forms from a minimal amount of NBs (Urbach and Technau, 2003b). 


\subsubsection{Neuroblast identity}

In the VNC, each NB generates a unique cell lineage and the identity of the individual NB is specified by both temporal and spatial pattern. The expression of the anteroposterior and dorsoventral patterning genes subdivide the ventral neuroectoderm into a grid like pattern. Anteroposterior patterning is mediated by the segment polarity genes, which are expressed in transverse stripes within each segment. Dorsoventral patterning is mediated by the the homeobox genes ventral nervous system defective ( $v n d)$, intermediate neuroblasts defective (ind) and muscle segment homeobox gene ( $m s h$ ) which are expressed along the DV axis in columns within each segment (Skeath, 1999). The NBs which arise in the corresponding position of each hemisegment are homologs. These homologs express the same combination of the developmental genes and generate similar cell lineages, which are modulated by the action of the Hox-cluster genes (McGinnis and Krumlauf, 1992; Urbach and Technau, 2003a; Urbach and Technau, 2004; Karlsson et al., 2010).

In Drosophila, the brain NBs are continuously added in a reproducible pattern. More than 40 molecular markers, including proneural genes, segment polarity genes, dorsoventral patterning genes and many others were used to establish a map of brain NBs for embryonic stages (Figure 2.5; Urbach and Technau, 2003b). This study revealed that each NB expresses a specific combination of transcription factors, and it was suggested that these combinations determine the individual fate of each NB. This complex co-expression pattern presumably reveals part of the mechanism for the specification of individual NBs and the components of their corresponding cell lineages, but the genetically relevant networks are still poorly known. Moreover, the segmental pattern is less conserved in the anterior brain. Serially homologous NBs have been found in the hemisegments of the VNC and the posterior brain (deuterocerebrum and tritocerebrum). However, the protocerebrum comprises very little homology to trunk neuromeres (Urbach and Technau, 2004). 


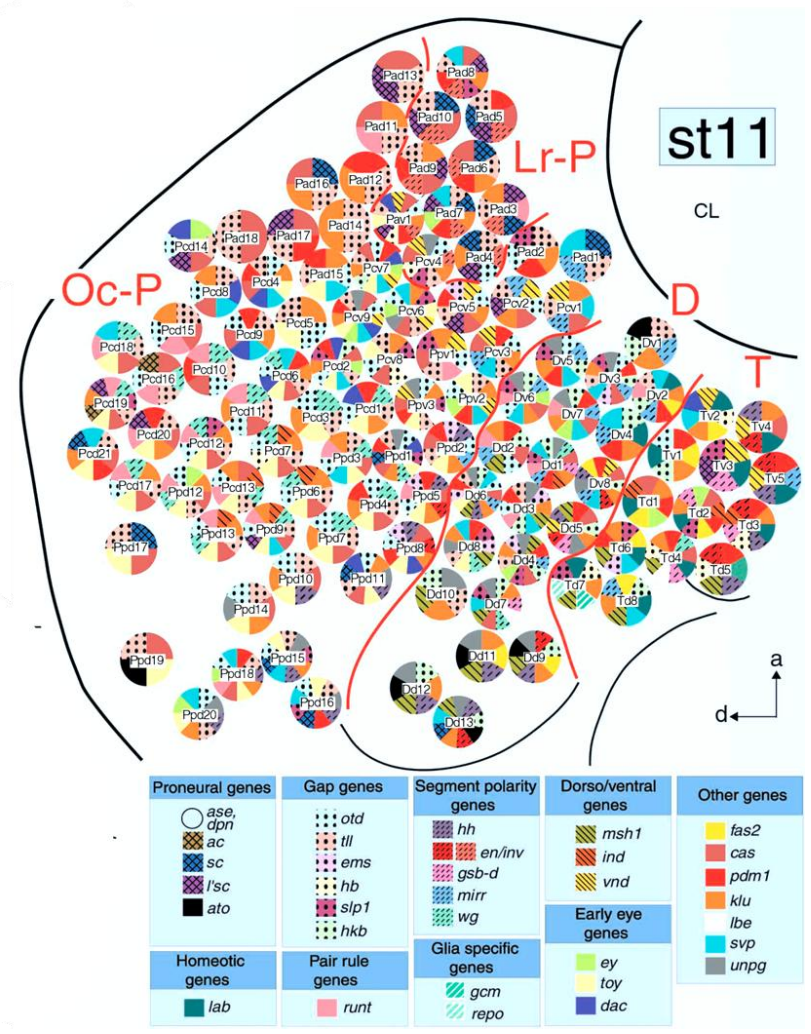

Figure 2.5 Specific combinations of marker gene expression reflect individual identities of brain NBs in Drosophila. More than 40 molecular markers (representing 34 different genes as listed below) have been found to specifically express in brain NBs. Each brain NB reveals a unique combinatorial code of marker gene expression. Red lines indicate the segmental boundaries between the protocerebrum (P), deutocerebrum (D) and tritocerebrum and (T). Lr-P: labral protocerebrum; Oc-P: ocular protocerebrum. (Taken from Urbach and Technau, 2003b)

\subsubsection{Neuroblast Lineages - type I and type II}

NBs are the primary progenitors which undergo multiple divisions to generate the unique set of neural cells forming a neural lineages consisting of neurons, glial cells and axonal fascicles. The cell bodies of a given lineage stay together to form a stereotypic cluster and make the similar projection patterns (Larsen et al., 2009; Yang et al., 2013; Lee, 2017). Recent studies have shown that there are two different types of NBs existing in the insect brain: type I NBs and type II NBs (Figure 2.6). Type I NBs generate relatively simple cell lineages and represent the classical mode of neurogenesis. They undergo asymmetric cell divisions to self-renew and bud off ganglion mother cells (GMCs). The GMC then divides symmetrically to produce two daughter cells that develop into neurons and/or glia (Truman and Bate, 1988; Doe, 2008; Knoblich, 2008). In Drosophila, type I NBs can be identified by certain molecular markers. Type I NBs express Deadpan (Dpn) and Asense (Ase) 
but not Pointed P1 (PntP1; Pnt) (Zhu et al., 2011; Xie et al., 2016). In contrast, type II NBs have more complicated and larger lineages. Type II NBs divide asymmetrically to self-renew and generate intermediate neural progenitors (INPs) which retain limited self-renewing potential. Each INP then divides to self-renew and produce a GMC which subsequently undergoes a terminal division to generate two neural progeny. Since each INP has the capacity to self-renew (four to eight times), the INPs have the potential to significantly amplify the number of the neural cells within the lineages. Type II NBs are positive for Dpn and PntP1 but not Ase and their progeny INPs express Dpn and Ase (Bello et al., 2008; Boone and Doe, 2008; Izergina et al., 2009; Weng and Lee, 2010; Zhu et al., 2011; Walsh and Doe, 2017).

A Type I neuroblast

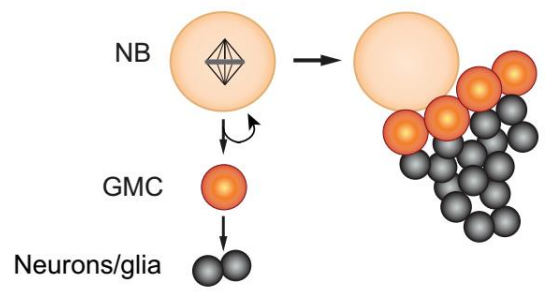

B Type II neuroblast

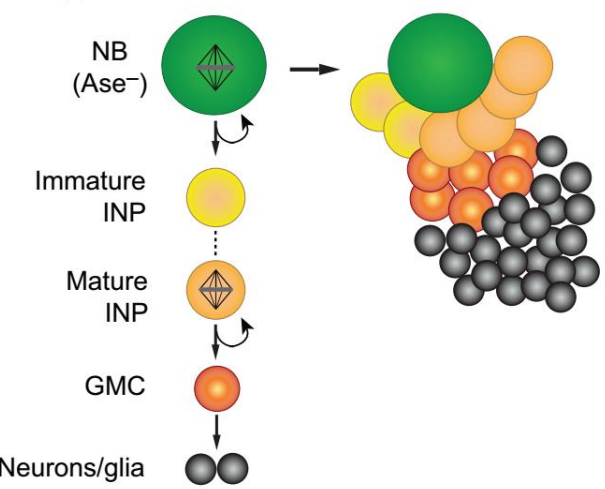

Figure 2.6 Type I NBs and type II NBs and their mode of asymmetric cell division. (A) Type I NBs divide asymmetrically to self-renew and generate a ganglion mother cell (GMC, orange). GMCs divide once to generate a pair of neurons or glia (gray). (B) Type II NBs divide asymmetrically to self-renew and generate an immature intermediate neural progenitors (INPs; yellow) which then divide asymmetrically to self-renew and to generate a GMC. The GMCs subsequently undergoes a terminal division to generate two neural progeny (gray). (Taken from Homem and Knoblich, 2012)

Most of the NBs in the insect brain are type I NBs. In the Drosophila embryonic brain, there are approximately 100 type I NBs per hemisphere, whereas only 8 type II NBs are identified (Walsh and Doe, 2017). Type I NBs usually give rise to the progeny contributing to the small and diffuse neuropils or to the mushroom bodies, whereas type II NBs generate larger lineages through amplifying proliferation of the intermediate progenitors, contributing to the central complex. For instance, in Drosophila type I NBs generate the intrinsic cells which contribute to the mushroom bodies, containing an average of approximately 150 to 200 cells in adult brain, whereas the dorsomedial (DM) lineages which derive from type II NBs and innervate to the central complex, have an average of 450 cells (Bello et al., 2008; 
Izergina et al., 2009; Pereanu et al., 2010). Similar findings have been also reported in grasshopper. The studies in the grasshopper show that the identified embryonic lineages $W, X, Y, Z$ contributing to the central complex are the largest lineages (Type II lineages) in the brain. Each of these type II lineages contains more progeny, around 4 to 5 times, than those associated with the embryonic mushroom bodies (Boyan and Williams 1997; Williams et al., 2005; Williams and Boyan 2008; Boyan et al., 2010). Some type I NBs, such as mushroom body NBs, form during embryogenesis and survive into larval stage or even adult stages, generating progeny persistently throughout development. This demonstrates they undergo long time proliferation (Cayre et al., 1996; Prokop and Technau, 1994; Ito et al., 1997; Kunz et al., 2012). By contrast, type II lineages are always generated during a restricted and shorter developmental period. For example, the dorsomedial (DM) lineages are generated at the late third larval stage in Drosophila (Bello et al., 2008) and $W, X, Y, Z$ lineages are generated at the mid-embryogenesis in Schistocerca (Boyan and Williams 1997; Williams et al., 2005; Boyan et al., 2010). These lineages do not increase significantly after that.

\subsection{Development of insect brain from embryo to adult}

The holometabolous insects develop larval morphology during embryogenesis and later undergo an extensive morphological transformation of the larva into flying adult during metamorphosis. How does the embryonic pattern develop into the adult brain? Insect brain development begins with the delamination of NBs from the anterior neuroectoderm. Following delamination, these brain NBs start generating their neuronal progeny (Hartenstein and Campos-Ortega, 1984). The brain NBs can be identified based on their stereotyped position and can also be characterized by the expression of cell-specific molecular markers (Zacharias et al., 1993; Urbach and Technau, 2003b). By late embryogenesis the embryonic NBs cease to proliferate and enter into quiescence, and the quiescent NBs reactivate to resume proliferation during the first larval stage. This postembryonic neurogenesis continues throughout larval stages and extends into pupal stages, giving rise to more than $90 \%$ of neurons contributing to the adult brain. At some time point, the NBs exit from the cell cycle and die (Truman and Bate, 1988; Prokop and Technau, 1991; Ito and Hotta, 1992; Maurange et al., 2008). The formation of the adult brain involves massive postembryonic neurogenesis and reorganization of a small portion of the neurons born during embryogenesis. Therefore, the NBs responsible for the larval neurogenesis are embryonic NBs that are reactivated postembryonically, generate the vast majority of neurons in the adult brain. 


\subsection{Development of brain commissures and the central}

\section{complex}

The axongenesis in the insect brain has been investigated in Drosophila and Schistocerca. At a certain stage during embryogenesis, the brain hemispheres become linked to each other at the midline by cells arranged in a bridge-like pattern, and at the same time the primary axon extends across the midline, which is pioneered by the cells located near the medial edge of each hemisphere (Boyan et al., 1995a, b, c; Therianos et al., 1995). The establishment of the primary axonal projection across the brain midline is thus closely associated with the interhemispheric cellular bridge. Both neurons and glial cells have shown to be involved in this process (Boyan et al., 1995a, c; Therianos et al., 1995). With the primary axon guidance, the later emerging axons will follow this pathway and extend across the midline. During subsequent embryogenesis, this brain commissure differentiates further and grows rapidly in size to become the massive commissures (Therianos et al., 1995). In the adult grasshopper, the brain hemispheres are interconnected by over 70 commissures which are derived from the primary brain commissure during embryonic development (Boyan et al., 1993).

The CX is a midline spanning neuropil in the insect brain and its development has been characterized in some insect species, particularly in Drosophila and Schistocerca. In Drosophila, the CX is first detectable in the third instar larva, showing the immature protocerebral bridge and fan-shaped body (Schneider et al., 1993; Young and Armstrong, 2010). The CX has also been described as a series of interhemispheric commissures at this stage (Hanesch et al., 1989). Four dorsomedial lineages (DM1-4) which are generated by four identified type II NBs are shown to contribute to the developing adult CX (Izergina et al., 2009; Pereanu et al., 2011; Boyan and Reichert 2011; Riebli et al., 2013; Yang et al., 2013). In Schistocerca, the CX starts differentiation during mid-embryogenesis (Reichert and Boyan, 1997). Four NBs located in the pars intercerebralis in each hemisphere are shown to be involved in the CX development. These NBs give rise to stereotypic neural clusters which contribute the axon fascicles initially to the protocerebral bridge $(\mathrm{PB})$ and then to central body $(\mathrm{CB})$ via four discrete tracts $(\mathrm{w}, \mathrm{x}, \mathrm{y}, \mathrm{z})$ (Boyan and Williams, 1997; Williams et al., 2005; Williams and Boyan, 2008; Boyan et al., 2008; Boyan and Reichert, 2011). The corresponding W, X, Y, Z clusters and tracts are also found in the adult brain (Williams, 1975; Boyan et al., 1993; Vitzthum et al., 1996). Studies have also shown that the $C X$ is innervated by different neuronal cell types. For instance, tangential neurons have arborizations outside the $C X$ and tangentially project to a single subunit of the $C X$ (e.g. within the particular layers of the $\mathrm{FB}$ or the entire rings of the $\mathrm{EB})$, while columnar neurons interconnect the $\mathrm{PB}$ to $\mathrm{CB}$ and divide the $\mathrm{PB}$ and $\mathrm{CB}$ as regular columnar elements (Homberg 1985; Hanesch et al., 1989; Loesel et al., 2002; Boyan and Williams 2011; Phillips-Portillo 
2012; Heinze et al., 2013; Pfeiffer and Homberg 2014). The axonal projections of columnar neurons may also extend to the lateral accessory lobe or to the noduli (Pfeiffer and Homberg 2014). Therefore, columnar neurons play an important role in the communication between different subunits of the $C X$.

\subsection{Tribolium castaneum - a model organism for the central brain development}

Drosophila melanogaster and Schistocerca gregaria, are the most studied two insect model systems for the central brain development. In Schistocerca, embryonic brain development was analyzed by morphological studies like immunohistochemistry, BrdU incorporation and dye injection (Boyan and Williams 1997; Wiliams et al., 2005; Boyan et al., 2010; Boyan and Liu 2014). However, the molecular and genetic investigations were limited due to the lack of sequenced genome and the limited potential of the RNAi technique (Dong and Friedrich, 2005). Advances in molecular techniques and various genetic tools make the Drosophila as the preferred model organism to study the brain development (Urbach and Technau, 2003b; Younossi-Hartenstein et al., 2006). However, Drosophila also has the limitation that it shows derived mode of embryogenesis. Notably, the central complex is not detected during the embryonic stage. This indicates that Drosophila is not suitable for studying the embryonic signals required for $\mathrm{CX}$ development.

The red flour beetle Tribolium castaneum, a holometabolous insect, is a representative of the most diverse species on earth, i.e. the coleopterans (Grimaldi and Engel, 2005). It exhibits a more typical developmental process of the insects. Hence, it has become a comparative model organism in evolutionary developmental biology (Brown et al., 2009). Choosing Tribolium as model organism for studying brain development is mainly for several reasons. First, embryonic central brain development is more typical than seen in Drosophila. Specifically, the $C B$ partially forms during embryogenesis and the primordium of $C B$ is detected in the first instar larva (Posnien et al., 2011b; Koniszewski et al., 2016). Second, with respect to functional genetics Tribolium has more tools than most other insects apart from Drosophila. The genome has been sequenced (Richards et al., 2008), which facilitates studying gene function. Tribolium shows robust and systemic RNAi for all developmental stages. The dsRNA injection into hemolymph makes the knockdown spread to all cells of the injected animal and is even transmitted to the offspring of injected females (parental RNAi) (Brown et al., 1999; Bucher et al., 2002). Furthermore, transposon-mediated transgenesis (Berghammer et al., 1999), misexpression tools including heat shock-based gene misexpression (Schinko et al., 2012), and GAL4/UAS-based misexpression (Schinko et al., 2010) have been 
established. Moreover, the large-scale transposon mediated mutagenesis screen GEKU provides various enhancer trap lines marked with EGFP (Trauner et al., 2009). In addition, powerful in vivo imaging tools have been generated: a nuclear reporter line (Sarrazin et al., 2012) and the light-sheet-based live imaging (Strobl and Stelzer, 2014; Strobl et al., 2015). Finally, the unbiased large-scale iBeetle-Screen, a genome-wide RNAi screen, allows finding novel genes required for a process apart from the classical candidate gene approach (Schmitt-Engel et al., 2015). Hence, Tribolium castaneum is a good model to study the genetic mechanisms of embryonic central brain development, including $C X$ development.

\subsection{CRISPR/Cas9 as an approach to generate imaging}

\section{lines}

One prerequisite for investigating brain development is the marking of subsets of neural cells. The recently discovered CRISPR/Cas technology has emerged as a highly versatile and efficient tool for genome editing. CRISPR (clustered regularly interspaced short palindromic repeats)/Cas (CRISPR-associated protein) is originally from the adaptable immune mechanisms of bacteria and archaea, in which CRISPR-derived RNA (crRNA) guides Cas nucleases to specifically target and cleave the foreign DNA, such as viral DNA (Horvath and Barrangou, 2010; Gasiunas et al., 2012; Jinek et al., 2012). Today, the CRISPR system most commonly used for genome editing, derived from Streptococcus pyogenes, consists of a Cas protein (Cas9) and a single chimeric guide RNA (known as gRNA) which is the combination of the crRNA and tracRNA (trans-acting antisense RNA). The guide RNA contains a region of 20 nucleotides at its $5^{\prime}$ end which pairs with the target DNA and determines specificity (crRNA), and an invariable sequence at its $3^{\prime}$ end that is indispensable to bind with Cas9 nucleases (tracRNA). Cas9 nucleases acts as 'molecular scissors' that is directed by the gRNA to target the specific DNA sequence in the genome and make a double-stranded break (DSB). Additionally, the target recognition depends on the presence of PAM that is typically NGG, which is located downstream of the 20 nucleotides target sequence in the genomic target DNA. Hence only sequences that match $\mathrm{N}_{20} \mathrm{NGG}$ can be targeted by CRISPR/Cas9. The DSB usually occurs 3 nucleotides upstream of the PAM $\left(\mathrm{N}_{17}{ }^{\vee} \mathrm{N}_{3} \mathrm{NGG}\right.$ ) (Garneau et al., 2010; Jinek et al., 2012; Jiang et al., 2013; Sander and Joung, 2014). With this approach, almost any gene of interest in the genome can be specifically targeted. The applications of the CRISPR/Cas have been established in model organisms and it also in principle allows the genetic intervention in other species as well (Bassett et al., 2013; Gratz et al., 2013; Port et al., 2014; Gilles and Averof, 2014; Awata et al., 2015; Gilles et al., 2015; Zhu et al., 2015; Bi et al., 2016; Hammond et al., 2016; Li et al., 2016; Wang et al., 2016). 
CRISPR/Cas9 mediated cleavage of DNA typically undergoes two major mechanisms for DNA damage repair: non-homologous end joining (NHEJ) and homology directed repair (HDR) (Figure 2.7). In NHEJ, the broken ends are ligated to each other, resulting in the introduction of small insertions or deletions (indels) of various lengths at the break site. NHEJ thus provides an efficient way to disrupt gene function and can be used to mediate gene knockout. Additionally, in NHEJ exogenous linear DNA can also be ligated to the broken ends in random orientation with the absence of homologous sequences in the repair template, which is called homology-independent knock-in. HDR-mediated repair is based on inserting specific sequences into the target locus in the presence of an exogenously introduced repair template which has homology arms flanking the insertion sequence. HDR thus generates precise modifications known as homology-dependent knock-in. In living organisms, NHEJ and HDR mechanisms are ubiquitous. NHEJ occurs more frequently than HDR does and considered to be the predominant repair mechanism (Gilles and Averof, 2014).

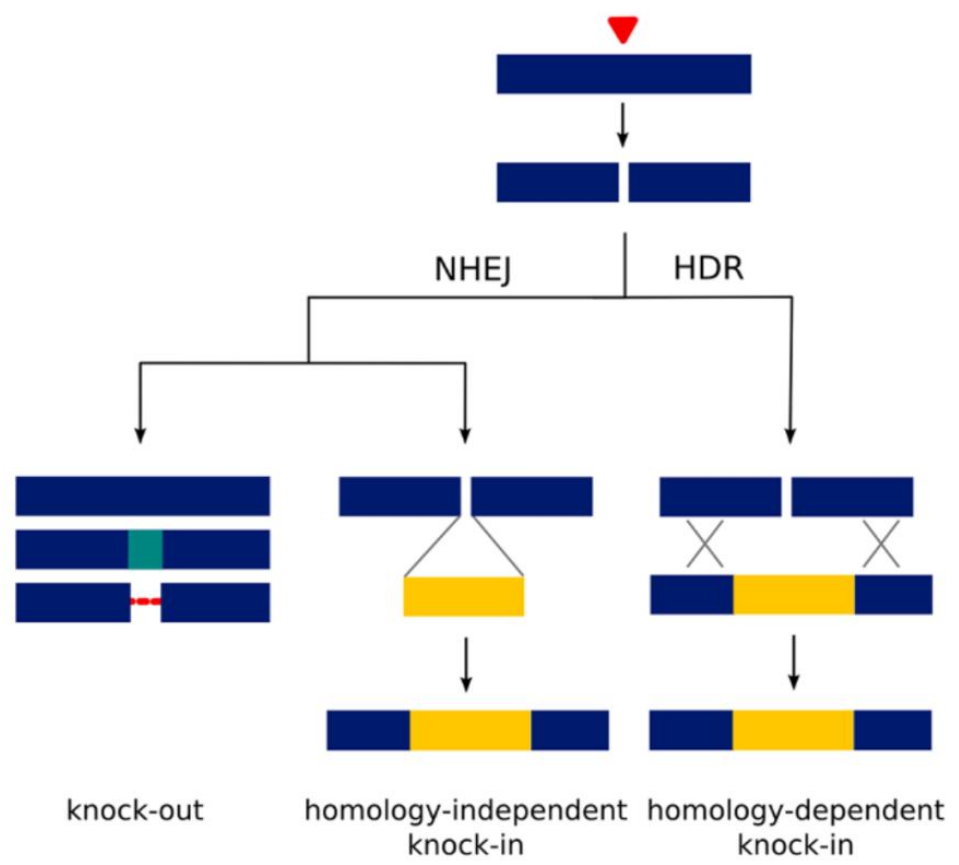

Figure 2.7 Schematic of repair mechanisms of CRISPR/Cas9 mediated double-stranded break. In non-homologous end joining (NHEJ), the broken ends are ligated, which results in either perfect rejoining or the introduction of small insertions (green) or deletions (red) at the break site. NHEJ can also introduce the exogenous linear DNA (yellow) to the broken ends. Homology directed repair (HDR) is based on inserting specific sequences into the target locus in the presence of an exogenously introduced repair template which has homology arms (blue) flanking the insertion sequence. (Taken from Gilles and Averof, 2014) 
The conventional approaches for generating imaging lines in Tribolium rely on the piggyBac transposon mediated integration of genetic constructs that are inserted randomly into the genome, such as using enhancer trapping (Trauner et al., 2009). Alternatively, one can use the regulatory region of the gene of interest to drive a reporter gene (Koniszewski, 2011) directly or via the Gal4/UAS system (Koniszewski et al., 2016). These lines prove to be good tools to study gene functions but have disadvantages: Enhancer trapping is a random process and many experiments are required to get one line that is interesting for a given project. Reporter constructs, in turn, often do not contain all regulatory elements and the expression depends on the genomic context. Hence, the patterns often do not closely match the endogenous expression. Compared with these approaches, apparently, CRISPR/Cas9 genome editing allows more efficient and easier solution by making an insertion at a specific location rather than a random one for specific research questions. In this study, I use CRISPR/Cas9 mediated NHEJ to generate a gene-specific enhancer trap line to mark neural cells throughout development for studying the NBs and their lineages which contribute to the central brain.

\section{$2.8 T c-f o x Q 2$ as a candidate gene for the central brain}

\section{development}

FoxQ2 is a member of forkhead transcription factor family. All members of the forkhead family are known to encode transcription factors, which share 110 amino acid encompassing DNA-binding domain, termed the forkhead domain (Kaufmann and Knöchel, 1996). The forkhead domain genes are found to be involved in development and differentiation (Kume et al., 2001; Mahlapuu et al., 2001; Zaffran et al., 2001). Some studies have uncovered that the forkhead domain genes play key roles in human health and diseases (Benayoun et al., 2011). FoxQ2 orthologs have been found in many species across the metazoan kingdom, including cnidarians, annelids, vertebrates and insects. Their comparable apical patternings in these different species suggest that foxQ2 is highly conserved among animals (Tosches and Arendt, 2013). Moreover, it has been shown that foxQ2 patterns a neurogenic region in Strongylocentrotus, Nematostella, Strigamia, Drosophila and Tribolium (Yaguchi et al., 2008; Sinigaglia et al., 2013; Hunnekuhl and Akam, 2014; Lee and Frasch, 2004; Kitzmann et al., 2017).

In protostomes, the function of foxQ2 has only been studied in Tribolium. The Tribolium ortholog Tc-foxQ2 is specifically expressed in the anterior head region and the corresponding neuroectoderm and it has proven to be an upstream factor within the anterior head gene regulatory network, with mutual activation of Tc-six3. Knock down of Tc-foxQ2 leads to a strong epidermal phenotype, showing the loss of the labrum (Kitzmann et al., 2017). The Drosophila ortholog of 
foxQ2 is $f d 102 c$. The embryonic expression pattern of $f d 102 c$ was characterized, showing a small anterior domain in blastoderm and a large number of neurons in the brain hemispheres at the late embryonic stage. However, the function of fd102c has not been studied (Lee and Frasch, 2004). In addition, foxQ2 function has been studied in sea urchin and sea anemone. In sea urchin Strongylocentrotus purpuratus, foxQ2 is involved in ectodermal patterning. Knock down of foxQ2 leads to a weak thickening of the animal plate ectoderm. But foxQ2 seems to be crucial for the development of serotonergic neurons (Yaguchi et al., 2008). In sea anemone, Nematostella vectensis, $N v$-foxQ2 is involved in the development of the aboral region. A defect of the outer morphology was not observed in $N v$-foxQ2 knockdown but the size of apical organ was reduced (Sinigaglia et al., 2013).

As head epidermis and brain originate from the same early neuroectodermal patterning in insects, the genes involved in head development are very likely to play an important role in brain development. In Tribolium, Tc-six3 is known to be an upstream regulator in anterior median head and required for the formation of the central body (Posnien et al., 2011b). Tc-foxQ2, mutually activated with Tc-six3 in anterior head patterning, has shown to be another key regulatory factor and the central brain is affected in knock-down animals. Knock-down of Tc-foxQ2 could result in reduction of the central body, convergence of the brain hemispheres and defects of the mushroom bodies in L1 Larvae (Kitzmann et al., 2017). However, the embryonic development of the anterior brain (protocerebrum) of the insect is poorly studied and the embryonic formation of the CX cannot be studied in Drosophila due to its postembryonic development. In order to get more insights into these processes, I used in this study $T c$-foxQ2 because it is exclusively expressed in the anterior protocerebral neuroectoderm, is required for anterior patterning in all animals and was shown to elicit central brain phenotypes.

\subsection{Aims of the study}

The insect brain is built by a conserved set of the neuropils which arise from the NBs. Each individual NB is specified by a combination of expressed transcription factors which are likely to determine its developmental fate. The progeny of a given NB form a stereotypic cell lineage. Therefore, the early determinants and signals that specify the NBs identities are essential for the formation of brain structures as well as their functions. The NBs identities are well studied for the trunk but poorly known for the brain.

In this study, I wanted to find out how many NBs express Tc-foxQ2 and I wanted to find out in how far their lineages contribute to the central brain and the central complex development. Further, I wanted to know, which transcription factors are co-expressed with Tc-foxQ2 to specify the NBs in the embryo. Finally, I intended to 
knock down Tc-foxQ2 by RNAi to study the phenotype in order to get insights into the function of $T c-f o x Q 2$ in embryonic brain development. As prerequisite for these studies, I generated a Tc-foxQ2 antibody and an imaging line to mark Tc-foxQ2 cells by using CRISPR/Cas9. With this imaging line, NBs and at least subsets of their lineages contributing to the central brain could be visualized and traced. 


\section{Material and Methods}

\subsection{Animals}

The San Bernadino (SB) wild type (wt) strain was used for cDNA synthesis, RNAi experiments, whole mount in situ hybridization and antibody staining.

The Tc-vermillion ${ }^{\text {white }}(T c-v w)$ strain was used for transgenesis. This line is a mutant of $T c-v w$ (tryptophan oxygenase) which results in non-pigmented eyes (white eyes) (Lorenzen et al., 2002). Eye marker expression can be easily screened within the $T c-v w$ strain.

The line E011A-01 (lab internal number: 50 ) is a Tc-rx enhancer trap line from the GEKU screen (http://www.geku.base.unigoettingen.de).

The line E035004 (lab internal number: 120) is an enhancer trap line derived from the GEKU screen and the insertion is in the Tc-ten- $a$ locus.

Tc-rx reporter line (lab internal number: 178) contains parts of the upstream genomic region of Tc-rx fused with basal promoter elements driving DsRedExpress (DsRedEx) (Koniszewski, 2011).

\subsection{Fixation}

\subsubsection{Fixation of the embryo}

The development from the zygote to the fully developed embryo lasts around $72 \mathrm{~h}$ at $32^{\circ} \mathrm{C}$ in Tribolium. Stock beetles were kept at $32^{\circ} \mathrm{C}$ for three days and $0-72 \mathrm{~h}$ aged embryos were sieved out and collected in $180 \mu \mathrm{m}$ mesh baskets. The embryos were rinsed with tap water several times and washed in $50 \%$ bleach twice for three minutes to dechorionate, afterwards they were thoroughly washed with tap water to completely remove the bleach. Fixation was performed using standard protocols (Schinko et al., 2009) as described with slight modifications. The fixation buffer here used is containing $6 \mathrm{ml}$ heptane, $2 \mathrm{ml}$ PEMS (0.1 M PIPES, $2 \mathrm{mM} \mathrm{MgCl}, 5 \mathrm{mM}$ EGTA, pH 6.9), and $300 \mu \mathrm{l} 37 \%$ formaldehyde. The embryos 
were fixed on a shaking platform at $225 \mathrm{rpm}$ for $25 \mathrm{~min}$. Fixed embryos were stored in methanol at $-20^{\circ} \mathrm{C}$ for in situ hybridization and antibody staining.

\subsubsection{Dissection and fixation of the larval brain}

The mid-late staged larva was selected and put into the ice-cold PBS. The larva was cut in the groove between the first thorax and second thorax by forceps. The cuticle of first thorax was removed, and then the cuticle of head was torn from lateral side, which resulted in the exposure of the brain. The dissected brains were collected in a $0.5 \mathrm{ml}$ tube filled with $375 \mu \mathrm{l}$ PBT (PBS with $0.1 \%$ Triton-X-100), which was kept on ice for the duration of dissection. The whole dissection procedure was taken no longer than $40 \mathrm{~min}$ to prevent brain degradation. For fixation, methanol-free formaldehyde was added to the final concentration of $4 \%$ to the tube. The brains were kept on ice for $30 \mathrm{~min}$, followed by washing with PBT. Next, antibody staining was performed (see section 3.3 ).

\subsubsection{Dissection and fixation of the adult brain}

The brains were dissected in ice-cold PBS by using forceps for a maximum of 40 min in case the brains started to degrade. The head was pulled off and placed dorsal side upwards. One forceps was used to hold the head by inserting the eyes, while the other forceps was used to lift the head capsule from the side close to thorax and expose the brain. The head capsule and tissues around the brain were carefully removed. The dissected brains were collected in a $0.5 \mathrm{ml}$ tube filled with $375 \mu$ l of PBT (PBS with $0.2 \%$ Triton-X-100), which was kept on ice for the duration of the dissection. For fixation, methanol-free formaldehyde was added to the tube, making the final concentration is $4 \%$. The brains were placed back on ice and kept for 45 min. Afterwards, the brains were washed with PBT and then were ready for the antibody staining.

\subsection{Immunohistochemistry}

\subsubsection{Antibodies}

The following primary antibodies were used: mouse-anti-ac.tubulin, chicken-anti-GFP, rabbit-anti-DsRed, rabbit-anti-Repo and guinea pig-anti-FoxQ2. The secondary antibodies included goat anti-mouse, goat anti-chicken, goat anti-rabbit and goat anti-guinea pig, coupled with Alexa Fluor 488 or 555, and with 
the dilution of 1:1000.

\subsubsection{Antibody staining of the embryo}

The fixed embryos were rehydrated by washing with PBT (PBS with $0.1 \%$ Triton-X-100) and incubated in blocking buffer (3\% BSA with $0.05 \%$ sodium azide in PBS) for $1 \mathrm{~h}$ at room temperature. Afterwards, the primary antibody was added and incubated overnight at $4^{\circ} \mathrm{C}$. After several times washing with PBT, the secondary antibody was added (dilution 1:1000) and incubated for $90 \mathrm{~min}$ at room temperature in the dark. Since then all the following steps were performed in the dark. The embryos were again washed with PBT, and 10 min DAPI staining ( $1 \mathrm{ng} / \mu \mathrm{l})$ was performed to stain the nuclei. The stained embryos were kept in VECTASHIELD ${ }^{\circledR}$ (Vector Laboratories) that is an antifade mounting medium for fluorescence and stored at $4^{\circ} \mathrm{C}$.

\subsubsection{Antibody staining of the larval brain}

After removing the PBT, the brains were incubated in blocking solution for $5 \mathrm{~h}$ at room temperature. The blocking solution was subsequently removed and the primary antibody, diluted in blocking solution, was added. The brains were incubated with primary antibody at $4^{\circ} \mathrm{C}$ overnight. Afterwards, the primary antibody was removed and the brains were washed with PBT for $3 \mathrm{~h}$ at room temperature. Then the secondary antibody was added in PBT with the dilution of 1:1000. From this step, the staining was performed in the dark. The brains were incubated with secondary antibody for $3 \mathrm{~h}$ at room temperature and rinsed with PBT, followed by a 15 min DAPI staining. After washing with PBT, a small amount of VECTASHIELD ${ }^{\circledR}$ was added to preserve the fluorescent staining. The samples were kept at $4^{\circ} \mathrm{C}$ for several hours. Then the brains were either mounted or transferred to $-20^{\circ} \mathrm{C}$ for longer storage .

\subsubsection{Antibody staining of the adult brain}

After removing the PBT, the brains were incubated in blocking solution at $4^{\circ} \mathrm{C}$ overnight. The blocking solution was subsequently removed and replaced by blocking solution with primary antibody. After incubation for $24 \mathrm{~h}$ at $4^{\circ} \mathrm{C}$, the primary antibody was removed and the brains were washed with PBT for $6 \mathrm{~h}$ at room temperature. Then the secondary antibody was added in PBT with the dilution of $1: 1000$. From this step, the staining was performed in the dark. The brains were incubated with secondary antibody overnight at $4{ }^{\circ} \mathrm{C}$ and rinsed with 
PBT, followed by a 30 min DAPI staining. After washing with PBT, a small amount of VECTASHIELD ${ }^{\circledR}$ was added. The samples were kept at $4^{\circ} \mathrm{C}$ for several hours. Then the brains were either mounted or transferred to $-20^{\circ} \mathrm{C}$ for longer storage .

\subsection{Fluorescent in situ hybridization}

\subsubsection{Probe}

RNA probes for in situ hybridization were synthesized with the DIG (Digoxegenin-UTP; DIG) RNA labeling mix (Roche, Germany) and the Fluorescin (Fluorescin-UTP; FLUO) labeling mix (Roche, Germany) using the T7 RNA polymerase. All RNA probes used in this thesis are available in the laboratory.

\subsubsection{Staining}

Fluorescent in situ hybridization was performed using a horseradish peroxidase (POD) mediated tyramide signal amplification (TSA). Staining was based on previously described protocol (Oberhofer et al., 2014) and was optimized by Marita Buescher. Finally, DAPI staining was performed.

\subsection{Mounting}

The stained embryos were submerged in VECTASHIELD ${ }^{\circledR}$ on a hollow microscopic slide. Yolk cells were removed from germ bands as much as possible. The embryos in different stages were transferred in a small drop of VECTASHIELD ${ }^{\circledR}$ between the lower slide and upper coverslip with spacers (upper coverslip $22 \times 22 \mathrm{~mm}$, lower slide $76 \times 26 \mathrm{~mm}$ ), carefully flattened and sealed with nail-polish. Staging of the embryos was carried out according to Biffar as a reference system (Biffar, 2014). The embryos from different developmental stages (NS3 to NS15) were selected and mounted to make the microscope slides. The slides were stored at $4^{\circ} \mathrm{C}$ overnight or $-20^{\circ} \mathrm{C}$ for longer time.

The stained larval brains and adult brains were mounted in a drop of VECTASHIELD ${ }^{\circledR}$ under a coverslip separated by spacers of $\sim 200 \mu \mathrm{m}$ thickness, so that the brain is not flattened. The slides were stored at $4^{\circ} \mathrm{C}$ overnight or $-20^{\circ} \mathrm{C}$ for longer time. 


\subsection{RNA interference (RNAi)}

The DNA templates for non-overlapping double-stranded RNA (dsRNA) fragments were produced by standard PCR from a plasmid template described previously (Kitzmann et al., 2017). The dsRNA was synthesized using the Ambion $^{\circledR}$ MEGAscript ${ }^{\circledR}$ T7 kit (Life Technologies, Carlsbad, CA, USA). The transcribed dsRNA was extracted via $\mathrm{LiCl}$ precipitation and dissolved in injection buffer $(1.4 \mathrm{mM} \mathrm{NaCl}$, $0.07 \mathrm{mM} \mathrm{Na}_{2} \mathrm{HPO}_{4}, 0.03 \mathrm{mM} \mathrm{KH}_{2} \mathrm{PO}_{4}, 4 \mathrm{mM} \mathrm{KCl}, \mathrm{pH}$ 6.8). The annealing of dsRNA was performed in a $94^{\circ} \mathrm{C}$ heating block for 5 min followed by slowly cooling it down to the room temperature. The concentrations of the injected dsRNA were measured using NanoDrop 1000 Spectrophotometer (constant 45 settings). Parental RNAi was performed according to previously published protocols (Bucher et al., 2002) by injecting dsRNA into pupae using the FemtoJet ${ }^{\circledR}$ express device (eppendorf, Germany). Different concentrations of dsRNA were used $(1.5 \mu \mathrm{g} / \mu \mathrm{l}$ and $3 \mu \mathrm{g} / \mu \mathrm{l}$ ) based on the requirement of experiment.

\subsection{Generation of a Tc-FoxQ2 polyclonal antibody}

\subsubsection{Cloning}

The C-terminal part of Tc-foxQ2 was amplified from cDNA pool by PCR using primer pairs with Bsal restriction site forward and reverse. PCR product was digested with $B s a l \mathrm{HF}$ and ligated into $\mathrm{pET}$-SUMO vector. $\mathrm{PET}$-SUMO and insert here were used at 1:5 molar ratio. Afterwards, the ligation was transformed into $E$. coli $\mathrm{DH} 5 \alpha$ cells. Positive clones were selected by colony PCR and confirmed by sequencing.

Ligation program in thermocycler:

$37^{\circ} \mathrm{C} 15 \mathrm{~min}$

$20^{\circ} \mathrm{C} 15 \mathrm{~min}$

Go to 1 repeat $20 x$

Hold at $14^{\circ} \mathrm{C}$

\subsubsection{Protein expression}

The BL21 (DE3) E. coli strain was used as the host for protein expression. The recombinant plasmid was isolated and transformed into BL21 (DE3). Single colony was inoculated into $25 \mathrm{ml} \mathrm{LB}$ medium containing $50 \mu \mathrm{g} / \mathrm{ml}$ kanamycin. The bacteria were grown overnight at $37^{\circ} \mathrm{C}$ with shaking $225 \mathrm{rpm}$. $1 \%$ bacterial culture was 
inoculated into $25 \mathrm{ml}$ TB buffer (with $1 \mathrm{x}$ phosphate and $50 \mu \mathrm{g} / \mathrm{ml}$ kanamycin) and grown at $37^{\circ} \mathrm{C}$ with shaking until the $O_{600}$ reached $0.6-0.8$ (approximately $3 \mathrm{~h}$ ). Then the culture was splited into two cultures: $20 \mathrm{ml}$ for IPTG induction and $5 \mathrm{ml}$ for control. 1M IPTG was added at 1:1000 into $20 \mathrm{ml}$ culture to induce expression. The two cell cultures were continued to incubate at $37^{\circ} \mathrm{C}$ with shaking for $4 \mathrm{~h}$. Afterwards, 2x $500 \mu \mathrm{l}$ aliquots from induced cell culture with IPTG and $500 \mu \mathrm{l}$ aliquot from uninduced cell culture were taken and centrifuged at maximum speed for $30 \mathrm{~s}$. Then cell pellets were kept and frozen at $-80^{\circ} \mathrm{C}$. Afterwards, protein expression and protein solubility were analyzed by sodium dodecyl sulfate polyacrylamide gel electrophoresis (SDS-PAGE).

\subsubsection{Protein purification}

The expression was scaled up to $1 \mathrm{~L}$ bacterial culture for purification. Bacterial pellet was resuspended in lysis buffer $(50 \mathrm{mM}$ Tris- $\mathrm{HCl} \mathrm{pH}=7.8,500 \mathrm{mM} \mathrm{NaCl}, 10$ $\mathrm{mM}$ imidazole) and fractionated by using Fluidizer (mechanical lysis, by high pressure $80 \mathrm{psi}$ ). After ultracentrifugation, the recombinant protein in supernatant was purified with $\mathrm{Ni}^{2+}$ chelate affinity chromatography which provides the very high affinity and selectivity for His-tags via gradient with $200 \mathrm{mM}$ imidazole in lysis buffer. Imidazole was able to compete for the coordination sites on $\mathrm{Ni}^{2+}$ and displace 6xHis. SUMO protease was used to cleave the His-SUMO tag, at the concentration of 1:50 with regard to the recombinant protein. Dialysis (50 mM Tris- $\mathrm{HCl} \mathrm{pH} 7.8,500 \mathrm{mM} \mathrm{NaCl}$ ) for removing imidazole and SUMO protease digestion were performed simultaneously overnight. Then the His-SUMO tag was separated from the protein of interest via re- $\mathrm{Ni}^{2+}$ chelate affinity chromatography. The flowthrough contained only the protein of interest. Gel-filtration chromatography (Superdex G-30) was performed to remove the remaining contaminations and finally the purified protein of interest was stored into PBS. All the steps for purification was done at $4^{\circ} \mathrm{C}$. Handling the instrument like fluidizer, centrifuge and Äkta, were following the instructor manual modified by Dr. Achim Dickmanns (AG Ficner), Kolja N. Eckermann and Max S. Farnworth in the laboratory.

\subsubsection{Antibody generation}

Purified C-terminal polypeptide of Tc-FoxQ2 (85 amino acids) was sent to Eurogentec (Liège, Belgium) for speedy 28 days polyclonal antibody production in guinea pig. To ensure no cross-reaction between the antigen and background antibodies, pre-immune screening of serum from five guinea pigs were analyzed and the best two guinea pigs were selected for immunization programme to raise antibodies against Tc-FoxQ2 polypeptide. The final serum is used straight as the Tc-FoxQ2 antibody. For antibody staining, anti-FoxQ2 was used as the primary 
antibody with the dilution of 1:1000 and anti-guinea pig coupled with Alexa Fluor 555 were used as secondary antibody.

\subsubsection{Antibody preobsorption}

Before antibody staining, preobsorption of anti-FoxQ2 was performed for eliminating non-specific binding. 0-12 $\mathrm{h}$ embryos were used for preobsorption as Tc-FoxQ2 expression was not detected during that stage. The embryos were fixed following standard protocol and incubated in blocking buffer for $1 \mathrm{~h}$. The blocking buffer was subsequently removed and replaced by anti-FoxQ2 diluted 1:1000 in blocking buffer. After incubation for $48 \mathrm{~h}$ at $4^{\circ} \mathrm{C}$, preobsorbed anti-FoxQ2 was pipetted in a fresh tube and stored at $4^{\circ} \mathrm{C}$.

\subsection{Generation of a $T c$-foxQ2 enhancer trap line by CRISPR/Cas9}

\subsubsection{Guide RNAs design and cloning}

The guide RNAs (gRNAs) were designed with the aid of the flyCRISPR Optimal Target Finder (http://tools.flycrispr.molbio.wisc.edu/targetFinder/; Gratz et al. 2014). The TriGenes gRNA oligo design tool was used for generating the sequences of the oligos to order. The annealed oligos were cloned into the gRNA expression vector $p$ (TcU6b-Bsal) via the Bsal restriction sites. The detailed annealing and ligation are following the protocol described previously (Gilles et al., 2015). Sequencing was performed with the T7-Minimal sequencing primer.

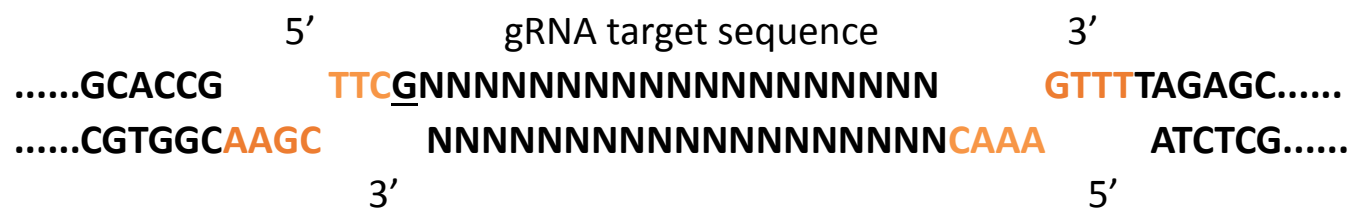

Figure 3.1 Detailed view of the gRNA expression vector $p(U 6 b-B s a l)$ cloning sites. The vector $\mathrm{p}$ (U6b-Bsal) with overhangs digested by Bsal and the gRNA target sequence designed with the complementary overhangs. The $G$ is the first nucleotide of $20-n t$ in target sequence marked in purple, which is necessary for U6 promoter driving expression. The orange sequence represents the overhangs generated by $\mathrm{Bsal}$ digestion. 


\subsubsection{Plasmids and cloning}

[3xP3-Tc'v-SV40; bhsp-EGFP-2A-Cre] is designed as a repair template for NHEJ-mediate knock-in by CRISPR/Cas9. For linearizing the plasmid, Dm-ebony target site (gRNA-eb) and Dm-yellow target site (gRNA-ye) were cloned into either side of the construct. Two different versions of repair plasmids were designed:

[3XP3-Tc'v-SV40-Cre-2A-EGFP-bhsp-gRNA-eb]

[gRNA-ye-3xP3-Tc'v-SV40-Cre-2A-EGFP-bhsp-gRNA-eb]

Each fragment of the construct was amplified by PCR from plasmids available in the laboratory's plasmid library by using primers with overhangs that are complements of two adjacent fragments. The 2A-peptide and the target sequences for cleavage were completely added by primers. In addition, an Apal and a Xbal restriction sites were added in end primers for the following ligation. Overlap extension PCR was performed to assemble all fragments together. The entire constructs were finally cloned into pJET1.2 vector. The repair templates were made by master student Dominik Mühlen.

The helpler plasmid $p$ (bhsp68-Cas9) expressing Cas9 was a gift from Michalis Averof (Addgene plasmid \# 65959).

\subsubsection{Guide RNAs testing}

T7 Endonuclease I assay was performed to test gRNA efficiency. Around 50 embryos aged 2-3 $\mathrm{h}$ were injected with an individual gRNA expression plasmid and Cas9 expression plasmid for a final concentration of $400-500 \mathrm{ng} / \mu \mathrm{l}$ each. The embryos were incubated at $32^{\circ} \mathrm{C}$ for two days. 20 survived embryos were transferred into $1.5 \mathrm{ml}$ tube and homogenized in $100 \mu$ buffer $(10 \mathrm{mM}$ Tris- $\mathrm{HCl} \mathrm{pH}$ 8.0, $25 \mathrm{mM} \mathrm{NaCl}, 1 \mathrm{mM}$ EDTA, $0.2 \%$ Triton-X-100 and freshly add $200 \mu \mathrm{g} / \mathrm{ml}$ Proteinase $\mathrm{K})$. The embryos were incubated at $55^{\circ} \mathrm{C}$ for $1 \mathrm{~h}$ and then at $95^{\circ} \mathrm{C}$ for 7-8 min. After centrifuge at $14000 \mathrm{rpm}$ for $2 \mathrm{~min}$ at room temperature, the supernatant was taken as the template for the following PCR reaction. Primers either side of the target sequence were designed to amplify a $\sim 1 \mathrm{~kb}$ region containing the target site by PCR (Table 7.1). After gel purification, 200 ng DNA mixing with $2 \mu \mathrm{l}$ 10xNEBuffer 2 and $\mathrm{H}_{2} \mathrm{O}$ to a total of $19 \mu \mathrm{l}$ were annealed by using a heating block, incubating at $98^{\circ} \mathrm{C}$ for $5 \mathrm{~min}$ and cooling slowly until to approximately $25^{\circ} \mathrm{C} .1 \mu \mathrm{l} \mathrm{T7}$ endonuclease I was added and incubated for $15 \mathrm{~min}$ at $37^{\circ} \mathrm{C}$. The reaction was stopped by adding $1.5 \mu \mathrm{l} 0.25 \mathrm{M}$ EDTA. Then the products were loaded on $1.5 \%$ agarose gel. 


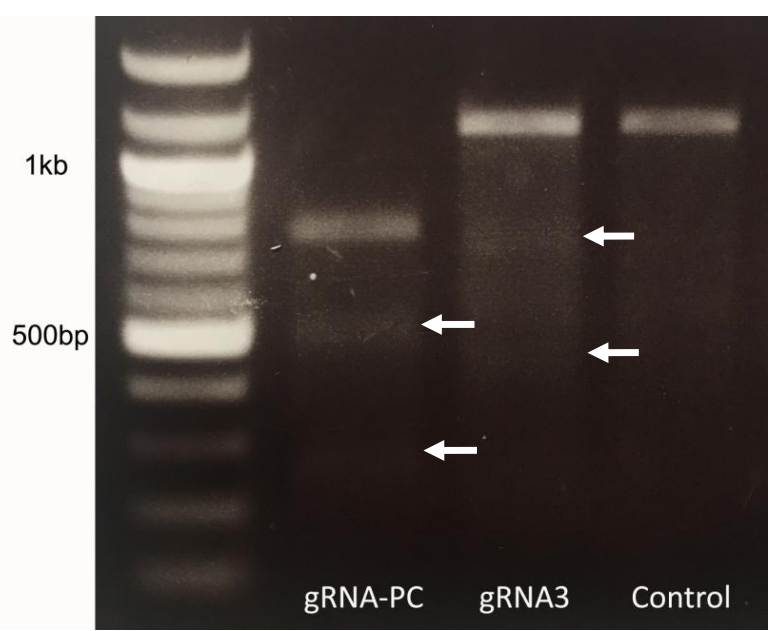

Figure 3.2 The efficiency of gRNAs tested by T7 Endonuclease I assay. gRNA-PC here is used as a positive control that works in Drosophila (from Hassan Mutasim Mohammed Ahmed ). gRNA3 targeting the first intron shows a smear and two additional cut bands but very weak. The size of the two bands is $\sim 750 \mathrm{bp}$ and $\sim 400 \mathrm{bp}$, which is consistent with the expectation. White arrow indicates the additional cut bands.

\subsubsection{Embryonic injection}

Tc-vw strain was used for transgenesis. Embryonic injection was performed according to standard procedure with little modification (Berghammer et al. 1999; Schinko et al., 2012). The beetles were transferred from whole grain flour at $32^{\circ} \mathrm{C}$ into white flour and kept at room temperature for $1 \mathrm{~h}$. The embryos were collected and further developed for $1 \mathrm{~h}$ at room temperature. Afterwards, embryos were washed with $1.5 \%$ bleach for $45 \mathrm{~s}$ followed by rinsing with tap water to get rid of residual flour and bleach. The embryos for injection were aged no more than $3 \mathrm{~h}$ at $25^{\circ} \mathrm{C}$. Before embryonic injection, all plasmids for CRISPR/Cas9 system need to be precipitated to remove the impurity and ensure the high enough concentration.

The final concentration of $p$ (bhsp-Cas9) and the repair plasmid is $500 \mathrm{ng} / \mu \mathrm{l}$ each, and $p$ (U6b-target-gRNA) is $125 \mathrm{ng} / \mu$ l each. $\mathrm{p}$ (U6b-gRNA-eb) and $\mathrm{p}$ (U6b-gRNA-ye) are used for linearizing the repair plasmid. Previous studies have shown that the used concentrations of Cas9 are range from 300 to $1000 \mathrm{ng} / \mu \mathrm{l}$, and the concentrations of gRNA are ranging from 75 to $500 \mathrm{ng} / \mu \mathrm{l}$, in which between 75 and $150 \mathrm{ng} / \mu \mathrm{l}$ are the optimal injection range (Ren et al., 2014; Gilles et al., 2015). Therefore, I used $125 \mathrm{ng} / \mu \mathrm{l}$ for gRNA and $500 \mathrm{ng} / \mu \mathrm{l}$ for Cas9, a concentration in between. This would not only avoid the potentially low efficiency due to the insufficient injection components but also ensure an appropriate survival rate.

Injected embryos were placed on apple agar plates in a sealed box at $32^{\circ} \mathrm{C}$ to keep 
them under humid conditions. After three days when the first larva hatched the box was opened and further incubated at $32^{\circ} \mathrm{C}$. Hatched larvae were collected and transferred as soon as possible into whole grain flour and kept at $32^{\circ} \mathrm{C}$.

\subsubsection{Screen}

The injected animals were separated into male and female during pupal stage. Each animal was crossed to three $T c-v w$ wild type beetles of the opposite sex. The beetles were kept at $32^{\circ} \mathrm{C}$ for several weeks in whole grain flour. The G1 offspring were screened for black eyes. Transgenic beetle was outcrossed with Tc-vw wild type and kept as a new stock.

\subsubsection{Establish the homozygous stock by genotyping}

Genotyping based on extracting genomic DNA from wing tissue was used to establish the homozygous stock. Individuals were sexed after pupation and separately reared at $32^{\circ} \mathrm{C}$ until they reached adulthood and were ready for wing dissection. Before dissection, individual adult was placed in a $1.5 \mathrm{ml}$ tube and kept on the ice for low temperature paralysis for $1 \mathrm{~min}$. Then the adult beetle was transferred to glass dish and held belly up with one forceps. The tip of other forceps was carefully inserted at the posterior region between the right elytron and the abdomen to slightly lift the elytron from the abdomen and expose the right wing. The wing was quickly dragged out as proximal as possible with forceps. The dissected wing was placed in a $200 \mu \mathrm{l}$ tube and kept on ice for the moment and the adult beetle was returned to the tube to recover from paralysis. The whole dissection process for one wing needs to be fast (no more than $30 \mathrm{~s}$ ), finished before the adult beetle woke up. One wing was dissected each adult beetle. After the dissection, the wings were frozen at $-80^{\circ} \mathrm{C}$ for $20 \mathrm{~min}$. Genomic DNA extraction from dissected wings was performed as previously described (Strobl et al., 2017), with slight modifications. $20 \mu \mathrm{l} \mathrm{HOM}$ buffer $(500 \mu \mathrm{g} / \mathrm{ml}$ Proteinase $\mathrm{K}, 10 \mathrm{mM}$ Tris- $\mathrm{HCl}, 1 \mathrm{mM}$ EDTA, and $25 \mathrm{mM} \mathrm{NaCl}$ in double-distilled $\mathrm{H}_{2} \mathrm{O}$ ) was added in each tube to cover the wing and a RNase-Free tip was used to physically homogenize the wing. The mixture was incubated at $37^{\circ} \mathrm{C}$ for $1 \mathrm{~h}$ and then inactivated at $75^{\circ} \mathrm{C}$ for $20 \mathrm{~min}$. From this solution, $1 \mu \mathrm{l}$ was used as a template for the PCR-based genotyping. Three primers were designed as a group for PCR amplification (Table 7.1) 


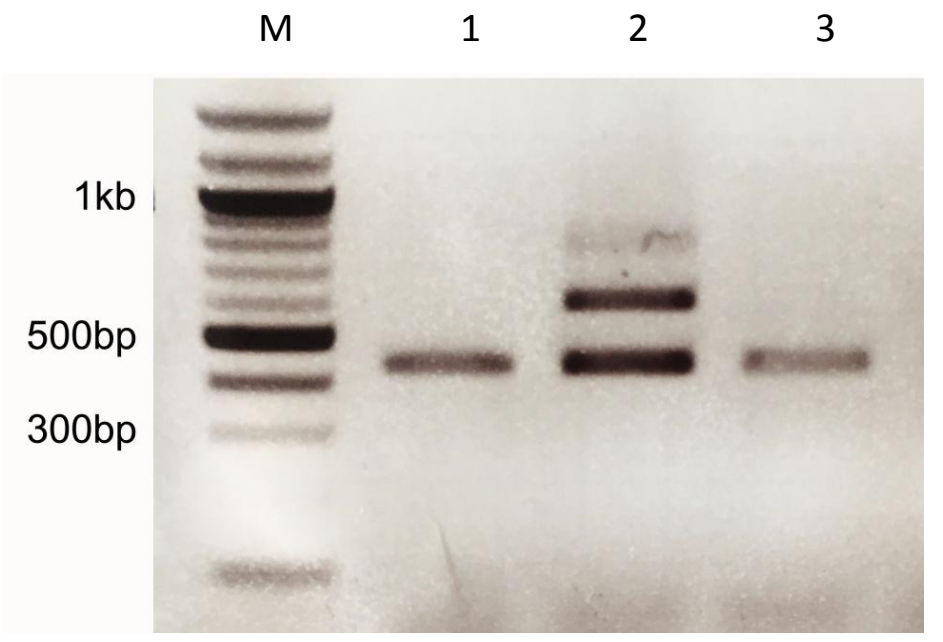

Figure 3.3 Agarose gel electrophoresis for the homozygous and heterozygous. The homozygous individuals would have two bands of estimated $606 \mathrm{bp}$ and $\sim 7.5 \mathrm{~kb}$ by PCR amplification, whereas the heterozygous individuals would have three bands of estimated $443 \mathrm{bp}, 606 \mathrm{bp}$ and $\sim 7.5 \mathrm{~kb}$. The estimated size of $\sim 7.5 \mathrm{~kb}$ was so large that I did not include it here. This was adequate to indentify the homozygous and heterozygous. Lane1 and Lane3, represent the homozygous individuals with one PCR product of $443 \mathrm{bp}$. Lane2, represents the heterozygous individual with two PCR products of $443 \mathrm{bp}$ and $606 \mathrm{bp}$.

\subsection{Image processing and documentation}

Immunohistochemistry, fluorescent in situ hybridization and DAPI staining were imaged using LSM510 (ZEISS). The best samples were selected and photographed with either the 20x or 40x objective. Z-projections were made using Fiji (Schindelin et al., 2012) with maximum intensity. All images were level-adjusted and assembled in Photoshop CS (Adobe). 


\section{Results}

\subsection{Generation of a Tc-FoxQ2 polyclonal antibody}

\subsubsection{Double fluorescent in situ hybridization leads to low resolution at the cellular level}

To identify Tc-foxQ2 positive NBs, Tc-asense (Tc-ase) a NB specific marker (Brand et al., 1993; Wheeler et al., 2003) was used. Double fluorescent in situ hybridization was performed. The co-expression of Tc-foxQ2 and Tc-ase was detected in the domain of anterior neuroectoderm (Figure 4.1A: white box). However, double in situ hybridization showed low cellular resolution, which led to the failure of the single cell identification. Although some cell might be identified with the presence of DAPI staining (Figure 4.1B, C: arrows), the results were still not convincing. Therefore, an antibody against Tc-FoxQ2 which allows significant advances in resolution and high quality needs to be generated.
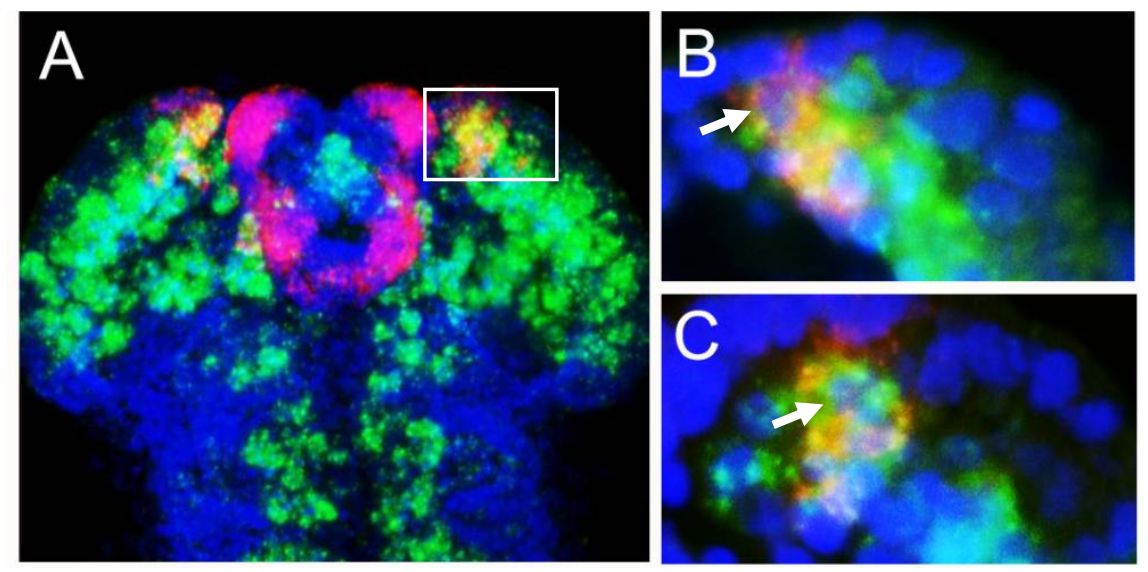

Figure 4.1 Double fluorescent in situ hybridization of $T c$-foxQ2 and Tc-ase in the embryos. (A) Overview of $T c-f o x Q 2$ and $T c$-ase expression. The co-expression was detected in the domain of anterior neuroectoderm (white box). (B, C) Close-ups of the single stack slice of the white box in (A). The arrows indicate the cells co-expressing $T c$-foxQ2 and $T c$-ase with low resolution, which leads to the failure of the cell identification. Red: Tc-foxQ2; green: Tc-ase; blue: DAPI. 


\subsubsection{Expression and purification of the Tc-FoxQ2 polypeptide}

For expression of Tc-FoxQ2 polypeptides, the pET SUMO Protein Expression System was used. This system is well known for significantly increasing the yield of difficult-to-express proteins in E. coli. Small ubiquitin-like modifier (SUMO) is the Saccharomyces cerevisiae Smt3 protein which is an $11 \mathrm{kDa}$ homolog of the mammalian SUMO-1 protein (Saitoh et al., 1997). The fusion of a protein to SUMO can increase its expression level as well as enhance the solubility of the recombinant protein. The tertiary structure of the SUMO protein can be specifically recognized and cleaved by SUMO Protease (Li and Hochstrasser, 1999; Mossessova and Lima, 2000). In addition, hexahistidine tag (6xHis-tag), which is able to bind to several types of immobilized metal ions, including nickel, cobalt and copper under specific buffer conditions is used for specifically detecting and purifying the recombinant fusion protein.

To avoid the cross-reactivity with proteins with high homology and make Tc-FoxQ2 antibody more specific, I excluded regions with high homology. Blast analyses in Tribolium suggested that 65 amino acids in the $\mathrm{N}$-terminal and 85 amino acids in the C-terminal region of Tc-FoxQ2 show little homology to other proteins (underlined in Figure 4.2).

MCSNETPANTSPRLPMPFALEGGPRALLPIDQYRLQLYQYAVAERLRYPLLNPFPTPL TCYPLFPRALQPEEPKPQHSYIGLIAMAILSSPEGKLVLSDIYQHILDHYPYFRTRGP GWRNSIRHNLSLNDCFIKAGRSANGKGHYWAIHPANVDDFRKGDFRRRKAQRKV RKHMGLAVDEDGADSPSPPPLSVSPPVVPGPSTSVYHTVPARGPSRKRQFDVASLL APDSGEDTNEEDIDVVSSDQHQETSPKQWPNMFPIVNYYQALLQARPGTTAETATD TTDS

Figure 4.2 Protein sequence of Tc-FoxQ2. The N-terminus containing 65 amino acids and the C-terminus containing 85 amino acids are underlined, which have little homology to other proteins in Tribolium.

The C-terminus of Tc-foxQ2 (Tc-foxQ2-C) was cloned into the pET SUMO vector that was modified by Prof. R. Ficner lab and K. N Eckermann. After induction with IPTG, high level expression of protein was detected (Figure 4.3A). Next, experiments were conducted to assess protein solubility. The cells were fractionated and after centrifugation, a large amount of protein was detected in the cell supernatant, indicating the protein was of high solubility (Figure 4.3B, lane 2). The size of Tc-FoxQ2-C polypeptide (85 amino acids) is $9.35 \mathrm{kDa}$ and expression of Tc-FoxQ2-C containing the $6 \mathrm{xHis}-\mathrm{SUMO}$ increases the size by approximately $13 \mathrm{kDa}$. Therefore, the expected size for recombinant fusion protein is $\sim 22 \mathrm{kDa}$. The recombinant fusion protein (6xHis-SUMO-Tc-FoxQ2-C) was purified with $\mathrm{Ni}^{2+}$ chelate affinity chromatography (Figure 4.3B, lane 4). With SUMO protease digestion, two bands were supposed to be detected at $\sim 13 \mathrm{kDa}$ (6xHis-SUMO) and $\sim 9 \mathrm{kDa}$ (Tc-FoxQ2-C). 
However, the size of both were larger than expected (Figure 4.3C, lane2: red arrows), which may be due to the slower migration during SDS-PAGE. Afterwards, by using re- $\mathrm{Ni}^{2+}$ chelate affinity chromatography, Tc-FoxQ2-C passed through and was collected in flow through (Figure 4.3C: red rectangle). Finally gel-filtration chromatography was performed to remove the remaining contaminations.

The N-terminal polypeptide of Tc-FoxQ2 was well expressed and was detected to be soluble as well. However, it had difficulties in purification, showing with very little amount of recombinant protein eluted by imidazole (data were not shown).

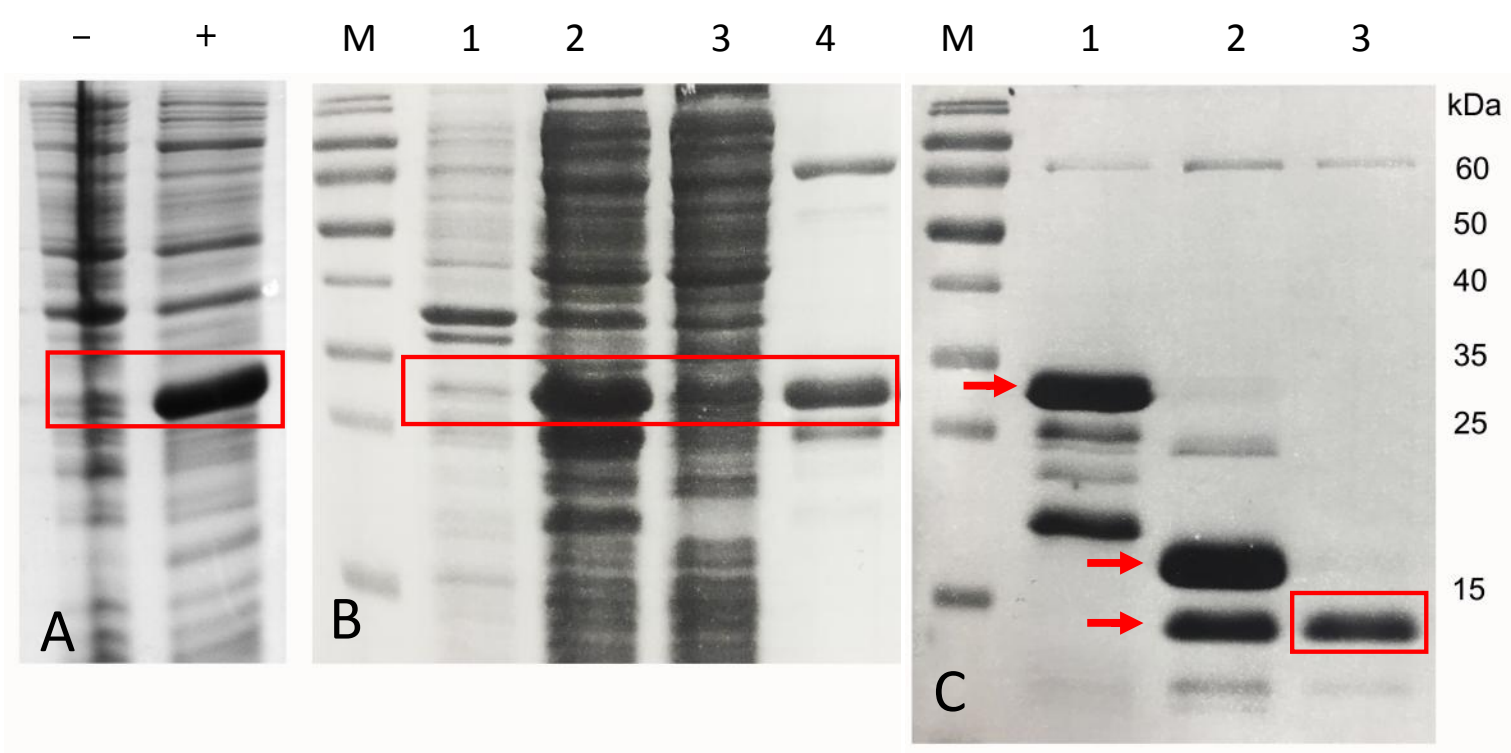

Figure 4.3 Comassie blue-stained SDS-PAGE gel analysis of expression and purification of Tc-FoxQ2-C. (A) Recombinant protein 6xHis-SUMO-Tc-FoxQ2-C expression induced by 1 mM IPTG for $4 \mathrm{~h}$ at $37^{\circ} \mathrm{C}$. (-) Before IPTG induction, as a negative control; (+) after IPTG induction. (B) Solubility analysis and purification via $\mathrm{Ni}^{2+}$ chelate affinity chromatography. M, marker; Lane 1, cell pellet; lane 2, supernatant; lane 3, flow through; lane 4, eluted fractions by $\sim 50 \mathrm{mM}$ imidazole. (C) SUMO protease digestion and re- $\mathrm{Ni}^{2+}$ chelate affinity chromatography. $\mathrm{M}$, marker; Lane 1 , before SUMO protease digestion, the estimated size of the recombinant protein 6xHis-SUMO-Tc-FoxQ2-C was $\sim 22 \mathrm{kDa}$ (red arrow); lane 2, after SUMO protease digestion, two bands were observed (red arrows), which were larger than expected size of $\sim 13 \mathrm{kDa}$ (6xHis-SUMO) and $\sim 9 \mathrm{kDa}$ (Tc-FoxQ2-C); lane 3, flow through after re- $\mathrm{Ni}^{2+}$ chelate affinity chromatography in which contained Tc-FoxQ2-C (red rectangle).

Purified C-terminal polypeptide of Tc-FoxQ2 was sent to Eurogentec (Liège, Belgium) for speedy 28 days polyclonal antibody production in guinea pig. The final serum was directly used as the Tc-FoxQ2 antibody. After concentration tests, I used 1:1000 dilution of anti-FoxQ2 as the primary antibody, and anti-guinea pig coupled Alexa Fluor 555 as secondary antibody for antibody staining. 


\subsubsection{Specificity of the Tc-FoxQ2 antibody}

To test the specificity of Tc-FoxQ2 antibody, in situ hybridization combined with antibody staining was performed in the embryos. As shown in Figure 4.4, from young to old stages, Tc-foxQ2 RNA showed a dynamic expression pattern (Figure 4.4A-E; Kitzmann et al., 2017), which was highly consistent with Tc-FoxQ2 protein expression (Figure 4.4A'-E'). Tc-foxQ2 expression was observed in two domains located at the anterior brain at the early stage (Figure 4.4A). Then the expression splitted into several domains, including non-neural expression in the labral and stomodeal domains (Figure 4.4C: dashed circle), as well as the expression domains in the neuroectoderm (Figure 4.4B-E: arrows). At late stages, the expression domains of Tc-foxQ2 in the neuroectoderm successively moved towards the midline along with morphogenetic movements of the head (Figure 4.4E: arrow; Kitzmann et al., 2017). A near complete overlap between Tc-foxQ2 RNA expression and Tc-FoxQ2 protein expression at all embryonic stages were observed (Figure $\left.4.4 A^{\prime \prime}-E^{\prime \prime}\right)$, suggesting high specificity of Tc-FoxQ2 antibody. DAPI staining was used to visualize the brain morphology during the embryonic development (Figure 4.4A'"'-E"').

Tc-foxQ2 RNA Tc-FoxQ2 protein

Merge

Merge/DAPI
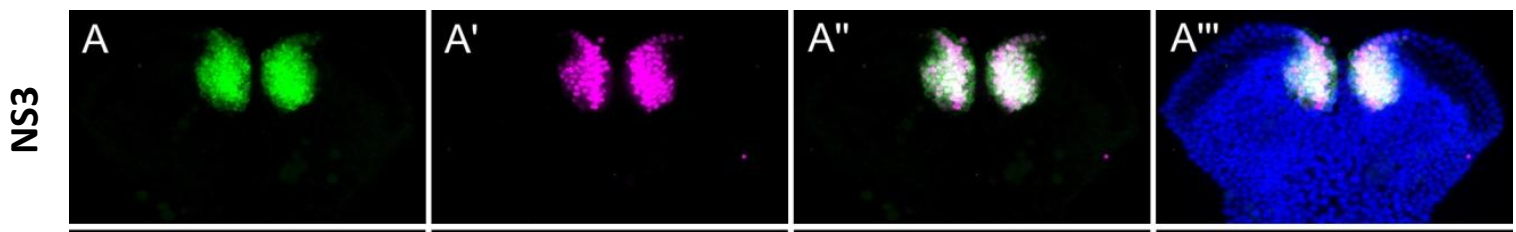

ก
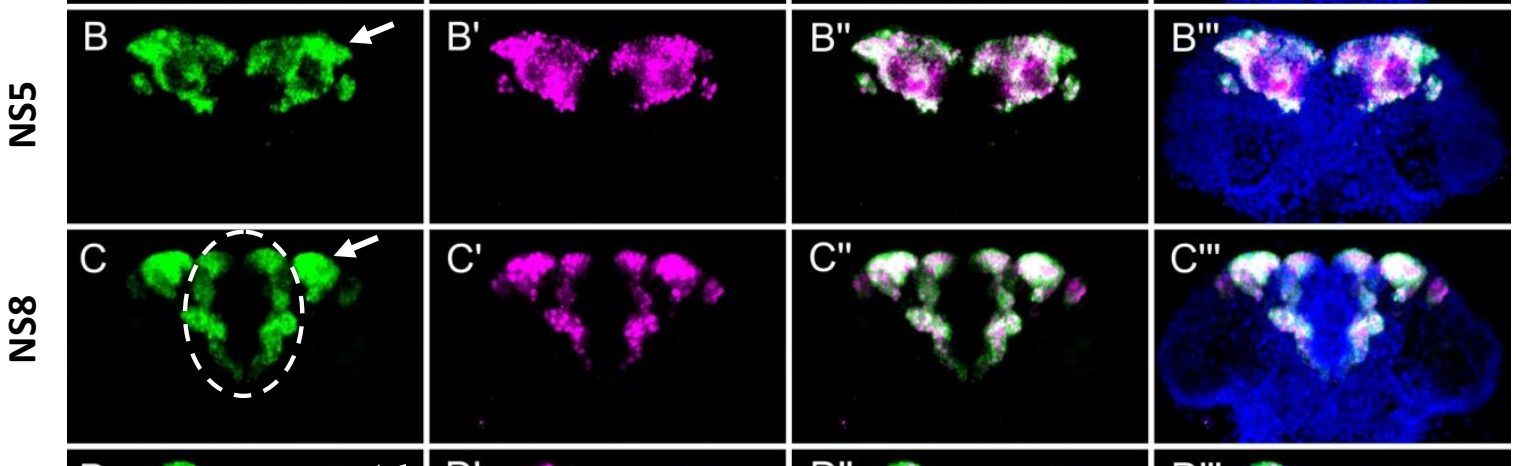

볼
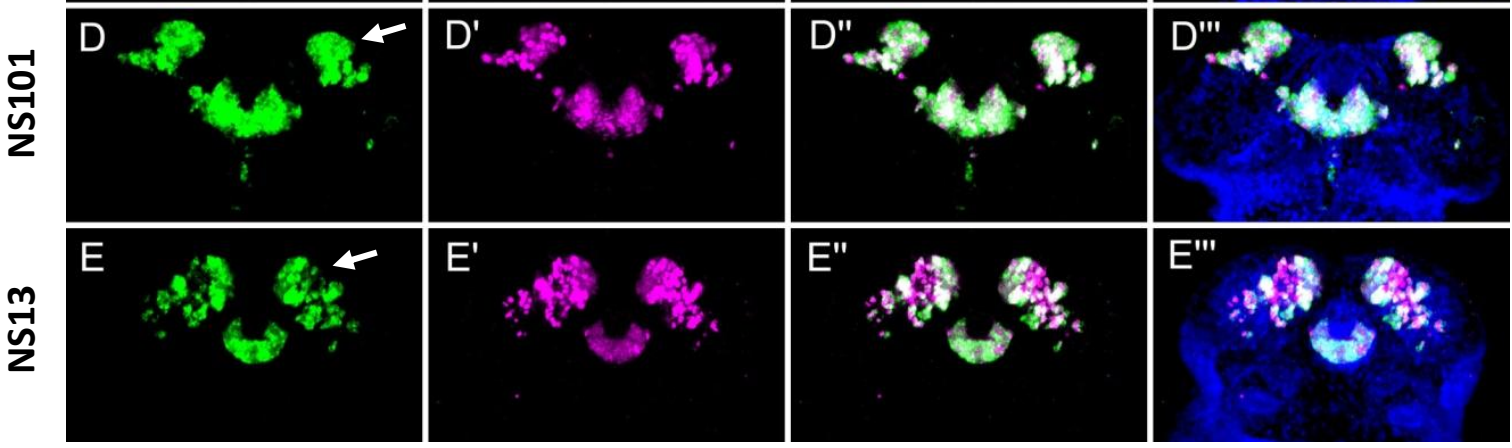

Figure 4.4 Overview of Tc-foxQ2 RNA expression and Tc-FoxQ2 protein expression from stage NS3 to NS13 in the embryos. (A-E) Tc-foxQ2 RNA shows a dynamic expression 
pattern in the anterior median region during the embryogenesis, showing in the putative neuroectoderm, labrum and stomodeum. ( $\left.A^{\prime}-E^{\prime}\right)$ Tc-FoxQ2 protein expression shows an identical expression pattern with Tc-foxQ2 RNA. ( $\left.A^{\prime \prime}-E^{\prime \prime}\right)$ Merge of Tc-foxQ2 RNA and Tc-FoxQ2 protein. ( $\left.A^{\prime \prime \prime}-E^{\prime \prime \prime}\right)$ Merge with nuclear staining DAPI to visualize the morphology of the embryo. Green: Tc-foxQ2 RNA; magenta: Tc-FoxQ2 protein; blue: DAPI.

At the cellular level, Tc-FoxQ2 protein exhibited much better resolution than Tc-foxQ2 RNA (Figure 4.5C). Tc-foxQ2 RNA was detected throughout the cytoplasm (Figure 4.5B), which made it difficult to identify cell boundaries and led to difficulties exactly determining the number of positive cells. Tc-FoxQ2 protein was observed in the nuclei which were stained with DAPI (Figure 4.5E) as expected because Tc-FoxQ2 is a transcription factor which is able to bind to DNA. Hence, using Tc-FoxQ2 antibody staining allowed a convincing cell identification.
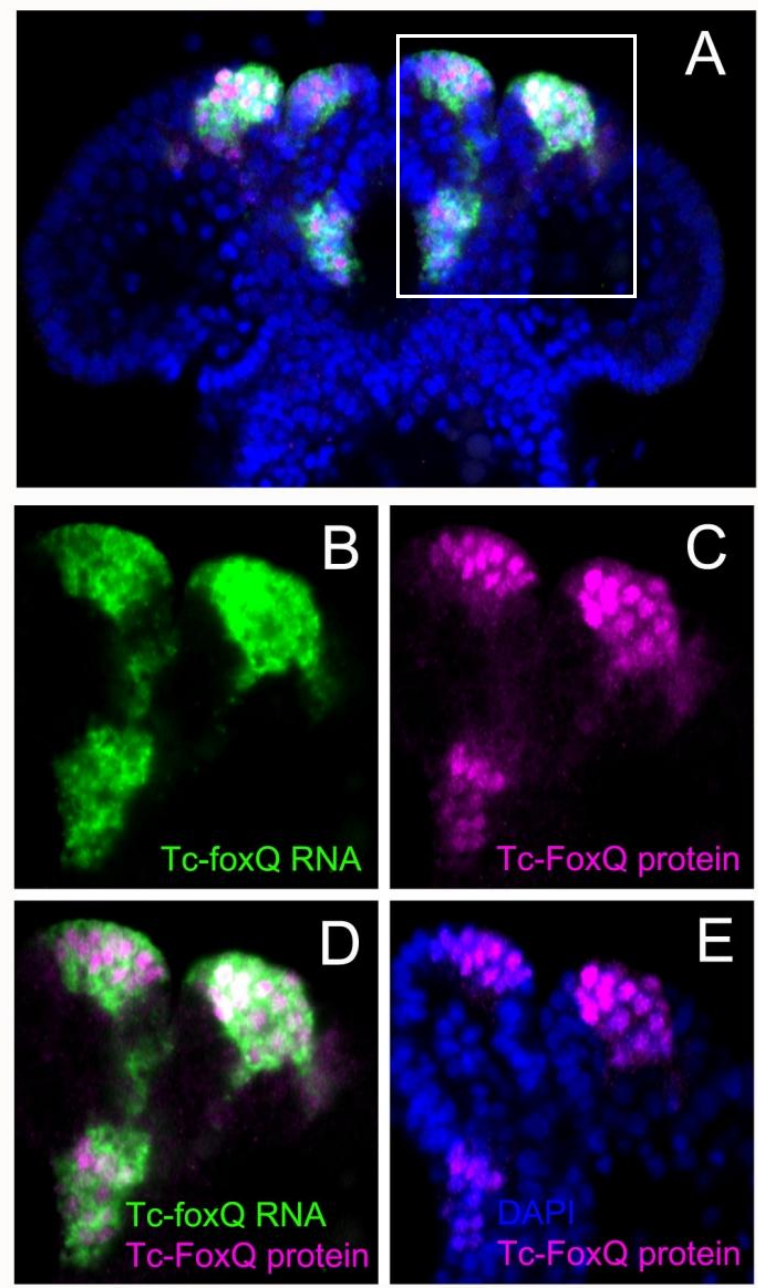

Figure 4.5 Single section of Tc-foxQ2 RNA expression and Tc-FoxQ2 protein expression. (A) Expression of Tc-foxQ2 RNA and Tc-FoxQ2 protein in the embryo. (B-E) Close-ups of the white rectangle in (A). (B) Tc-foxQ2 RNA is detected throughout the cytoplasm. (C, E) Tc-FoxQ2 protein is detected in the nuclei. (D) Tc-foxQ2 RNA and Tc-FoxQ2 protein shows a complete overlap. Green: Tc-foxQ2 RNA; magenta: Tc-FoxQ2 protein; blue: DAPI. 


\subsection{Generation of a $T c$-foxQ2 enhancer trap line by CRISPR/Cas9}

To detect Tc-foxQ2 positive cells throughout development, I generated an enhancer trap line in the Tc-foxQ2 locus via CRISPR/Cas9 mediated non-homologous end joining (NHJE). NHEJ, with the absence of the homology arms flanking the insertion sequence, provides a faster approach for gene knock-in. In NHEJ, the exogenous linear DNA can be ligated to the broken ends in random orientation, resulting in the introduction of insertion at the break site. Although NHEJ is more error prone compared to the homology directed repair (HDR) for knock-in, the target sites of the enhancer trap line are in the upstream region or the first intron of the gene, in which the precise approach is not required.

I designed the guide RNAs (gRNAs) targeting the upstream region or the first intron of Tc-foxQ2. The choice of gRNAs is often quite limiting and sometimes the gRNAs will have off-target properties. I selected the most promising gRNAs with the prediction of none off-targets or less off-targets. To increase the probability of a CRISPR/Cas9 mediated double stranded break (DSB), two gRNAs were designed to target the upstream region, and three to the first intron (Figure 4.6). The gRNA target sequence was cloned into the gRNA expression vector $p$ (U6b-Bsal) which contains Tribolium U6 promoter and tracrRNA sequences by Bsal restriction sites (Gilles et al., 2015).

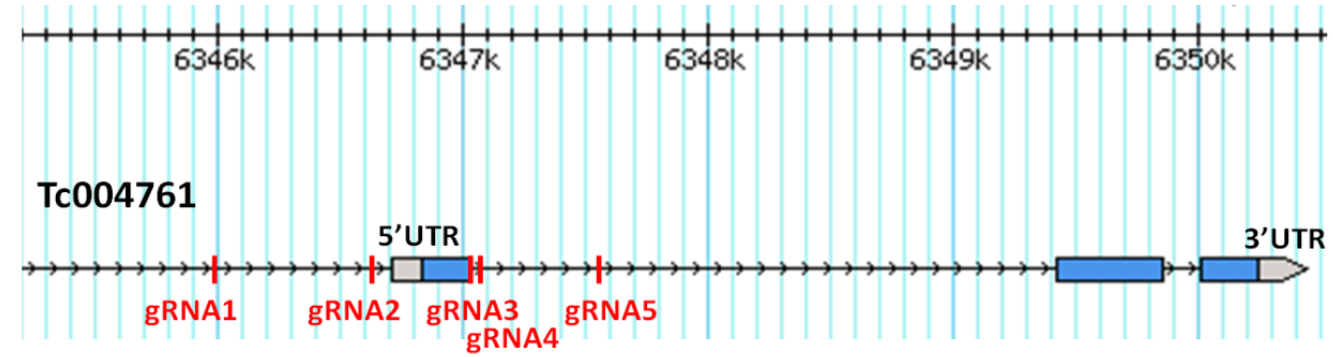

Figure 4.6 The genomic region of Tc-foxQ2 (Tc004761) and the location of the gRNAs target sites in genome. gRNA1 and gRNA2 target the upstream region. gRNA3, gRNA4 and gRNA5 target the first intron. The genomic region and sequence of Tc-foxQ2 were viewed by the iBeetle genome browser (http://bioinf.uni-greifswald.de/gb2/gbrowse/tcas5/).

The repair template was a multicistronic transgene which consisted of the enhanced green fluorescent protein EGFP and Cre recombinase linked by the $2 \mathrm{~A}$ peptide under the control of the basal heat shock promoter. $2 \mathrm{~A}$ peptide was self-cleaving, which was used to express multiple proteins from a single 
transcription unit through the cleavage of itself (Szymczak-Workman et al., 2012). The Cre recombinase allowed for the future use of the Cre/loxP system. This heat shock core promoter Tc-hsp68 was non-heat shock responsive and it did not drive the expression on its own but was able to be activated by the combination with enhancers (Schinko et al., 2012; Schinko et al., 2010). In addition, the repair template also contained the marker Tc-vermillion ${ }^{\text {white }}(T c-v W)$, which was driven by an eye-specific promoter $3 \times \mathrm{PP} 3$ and rescued the eye color in transgenic individuals within the Tc- $v w$ strain (white eyes switched to black eyes) (Berghammer et al., 1999; Lorenzen et al., 2002).

For the NHEJ-mediated knock-in, the repair plasmid had to be linearized. To avoid targeting of the endogenous genes at the same time, Drosophila target sequences Dm-ebony and Dm-yellow were chosen and cloned into the plasmid. The Drosophila target sequences selected were supposed to possess efficient targets of gRNAs so that the plasmid was linearized with a high probability (Ren et al., 2014). Furthermore, the Drosophila target sequences were also checked for homologous sequences in Tribolium to exclude endogenous gene targeting. Two different versions of the repair plasmids were designed. Both had a Dm-ebony target site (gRNA-eb) at the $5^{\prime}$ end of the heat shock promoter, whereas one had an additional Dm-yellow target site (gRNA-ye) at the $3^{\prime}$ end of the 3xP3 promoter (Figure 4.7A, B). The repair plasmid would be opened by the gRNA targeting sequence in itself. If the repair plasmid had both gRNA-eb and gRNA-ye target sites, it would be excised from the plasmid without backbone. If the repair plasmid only had gRNA-eb target site, it would be just linearized and inserted with backbone.
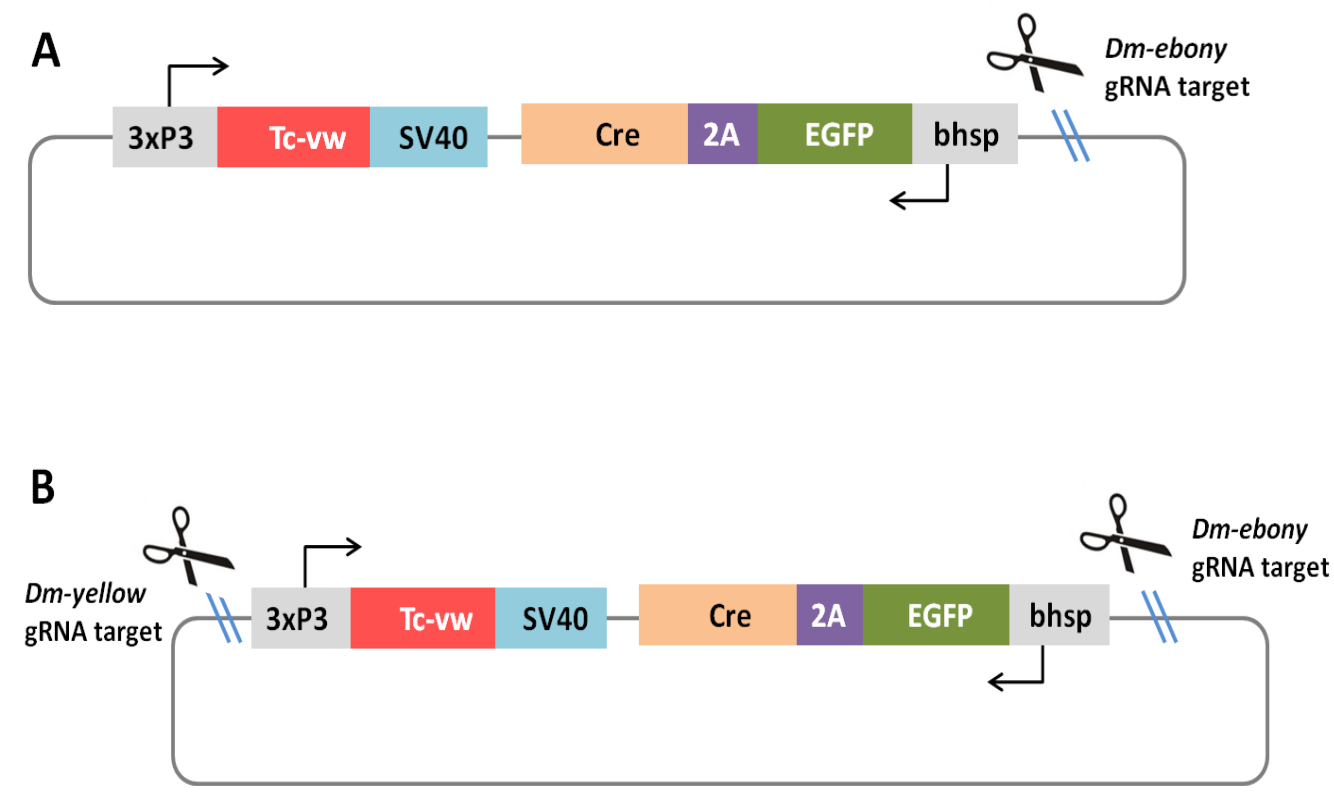

Figure 4.7 The scheme of the repair templates. (A) The repair template with Dm-ebony gRNA target site. (B) The repair template with both Dm-ebony and Dm-yellow gRNA target site. The basal heat shock promoter drives expression of EGFP and Cre which are 
connected by $2 \mathrm{~A}$-peptide. $T c-v w$ is a marker gene under the control of the $3 \times \mathrm{PP} 3$ promoter, followed by Sv 40 which serves as polyadenylation signal.

I injected all gRNAs targeting the same region (the upstream region or the first intron) together with the repair plasmid, Cas9 expression plasmid and the gRNAs for linearizing the repair plasmid. As two repair plasmids were designed and two regions were targeted, four injection combinations were performed (Table 4.1). The final concentration of $p$ (bhsp-Cas9) and the repair plasmid is $500 \mathrm{ng} / \mu \mathrm{l}$ each, and $p$ (U6b-target-gRNA) is $125 \mathrm{ng} / \mu \mathrm{l}$ each. For each combination, 1000 to 1600 embryos were injected to ensure the high number of survival animals. Developed pupae were separated into males and females and cross each with three $T c-v w$ wild type pupae of opposite sex and G1 animals were screened for black eyes.

Table 4.1 Components of the injection mix and concentrations for CRISPR/Cas9.

\begin{tabular}{|c|c|c|c|c|}
\hline Upstream & Intron1 & Upstream & Intron1 & $\begin{array}{c}\text { Final } \\
\text { concentration }\end{array}$ \\
\hline p(bhsp-Cas9) & p(bhsp-Cas9) & p(bhsp-Cas9) & p(bhsp-Cas9) & \multirow{2}{*}{$\begin{array}{c}500 \mathrm{ng} / \mu \mathrm{l} \\
\text { each }\end{array}$} \\
\hline $\begin{array}{l}\text { Repair plasmid } \\
\text { with eb target site }\end{array}$ & $\begin{array}{c}\text { Repair plasmid } \\
\text { with eb target site }\end{array}$ & $\begin{array}{l}\text { Repair plasmid with } \\
\text { eb and ye target site }\end{array}$ & $\begin{array}{l}\text { Repair plasmid with } \\
\text { eb and ye target site }\end{array}$ & \\
\hline p(U6b-gRNA-eb) & p(U6b-gRNA-eb) & $\begin{array}{l}p(\text { U6b-gRNA-eb) } \\
p(\text { (U6b-gRNA-ye) }\end{array}$ & $\begin{array}{l}p(\text { U6b-gRNA-eb) } \\
p(\text { U6b-gRNA-ye) }\end{array}$ & \multirow{4}{*}{$\begin{array}{c}125 \mathrm{ng} / \mu \mathrm{l} \\
\text { each }\end{array}$} \\
\hline $\mathrm{p}$ (U6b-gRNA1) & $\mathrm{p}($ U6b-gRNA3) & $\mathrm{p}(\mathrm{U} 6 \mathrm{~b}-\mathrm{gRNA} 1)$ & $\mathrm{p}($ U6b-gRNA3) & \\
\hline$p($ U6b-gRNA2) & $\mathrm{p}($ U6b-gRNA4) & $p($ U6b-gRNA2) & $\mathrm{p}($ U6b-gRNA4) & \\
\hline & $\mathrm{p}$ (U6b-gRNA5) & & $\mathrm{p}($ U6b-gRNA5) & \\
\hline
\end{tabular}

As shown in Table 4.2, the hatching rate of the injected embryos was more than $30 \%$ and the survival rate ranged from $14.14 \%$ to $21.88 \%$. The hatching rate of the repair plasmid without backbone was higher $(42.85 \%, 40.86 \%)$ than that of the repair plasmid with backbone $(31.99 \%, 32.67 \%)$. The adult survival rate showed the consistent results. Interestingly, the survival rate of the upstream treatment which contained two gRNAs was higher than the first intron treatment with three gRNAs. Finally, I got nine positive transgenic lines from the repair plasmid with backbone treatment, in which six lines from the upstream and three from the first intron. The efficiency of germ line transmission was therefore $2.6 \%$ and $1.3 \%$, respectively. However, I did not get any positive transgenic individuals from the repair plasmid without backbone treatment, neither the upstream nor the first intron. 
Table 4.2 The number of the injected embryos, the hatched larvae and developed adult beetles as well as the efficiency of germ line transmission.

\begin{tabular}{cccccccc}
\hline $\begin{array}{c}\text { Knock-in } \\
\text { repair } \\
\text { plasmid }\end{array}$ & $\begin{array}{c}\text { Target } \\
\text { region }\end{array}$ & $\begin{array}{c}\text { Injected } \\
\text { embryos }\end{array}$ & $\begin{array}{c}\text { Hatched } \\
\text { larvae }\end{array}$ & $\begin{array}{c}\text { Hatching } \\
\text { rate[\%] }\end{array}$ & $\begin{array}{c}\text { Developed } \\
\text { adults }\end{array}$ & $\begin{array}{c}\text { Survival } \\
\text { rate [\%] }\end{array}$ & $\begin{array}{c}\text { Efficiency } \\
\text { [\%] }\end{array}$ \\
\hline With & Upstream & 1496 & 479 & 31.99 & 234 & 15.64 & $6 / 234=2.6$ \\
\cline { 2 - 8 } backbone & Intron1 & 1619 & 529 & 32.67 & 229 & 14.14 & $3 / 229=1.3$ \\
\hline Without & Upstream & 1097 & 470 & 42.85 & 240 & 21.88 & $---{ }^{2}$ \\
\cline { 2 - 8 } backbone & Intron1 & 1303 & 532 & 40.86 & 251 & 19.26 & $-\ldots-$ \\
\hline
\end{tabular}

The nine transgenic lines were analyzed for EGFP expression. First, I checked the green fluorescence of the washed embryos from these lines under the fluorescence microscope. The three transgenic lines of the first intron showed little EGFP expression in anterior median head, which was not consistent with Tc-FoxQ2 expression. Among the six transgenic lines of the upstream region, only one line No.86 showed EGFP expression quite similar to Tc-FoxQ2 expression, the others exhibited either no EGFP expression or the EGFP expression in the unexpected parts of the body (Table 4.3). The multiply different patterns could be owing to the different orientation of the insertion or off-target effects. Further, since two gRNAs together were used to target the upstream region, this could lead to several possibilities, e.g. the insertion was in either gRNA1 or gRNA2, both gRNAs worked and the entire part between them was deleted. All these possibilities could probably lead to the different patterns. As I aimed at a functional Tc-foxQ2 line, I did not follow up these possibilities. Then antibody staining was performed in the embryos of the line 86up to compare EGFP expression and Tc-FoxQ2 endogenous expression. As expected, the expression of EGFP was very highly overlapping with Tc-FoxQ2 (Figure 4.8). Data were just shown the stage NS3 (A-A'"') and NS5 (B-B'"') here. More detailed descriptions of this line see section 4.4. The remaining CRISPR lines were not identified and described in this thesis due to the unexpected EGFP expression. 
Table 4.3 The initial analysis for the EGFP expression of the transgenic lines.

No. marks individual surviving $\mathbf{G O}$ animals.

\begin{tabular}{|c|c|}
\hline $\begin{array}{c}\text { Intron1 } \\
\text { No. }\end{array}$ & \multicolumn{1}{|c|}{ EGFP expression } \\
\hline 109 & $\begin{array}{l}\text { Little EGFP expression in anterior } \\
\text { median head }\end{array}$ \\
\cline { 1 - 1 } 125 & \\
\cline { 1 - 1 } 144 & \\
\hline
\end{tabular}

\begin{tabular}{|c|l|}
\hline $\begin{array}{c}\text { Upstream } \\
\text { No. }\end{array}$ & \multicolumn{1}{|c|}{ EGFP expression } \\
\hline 86 & $\begin{array}{l}\text { In anterior median head, } \\
\text { quite similar to Tc-FoxQ2 }\end{array}$ \\
\hline 129 & In the brain and trunk, not specific \\
\hline 150 & In the brain and trunk, not specific \\
\hline 165 & No EGFP signal \\
\hline 178 & In the part of brain and trunk \\
\hline 214 & In the posterior of abdomen \\
\hline
\end{tabular}

Tc-FoxQ2
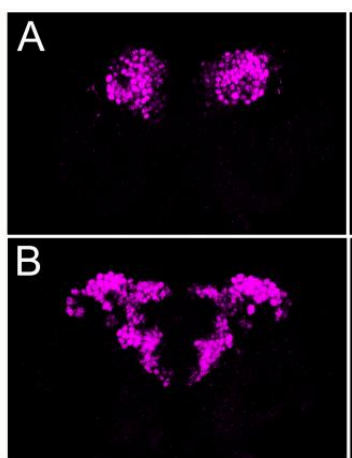

\section{EGFP}
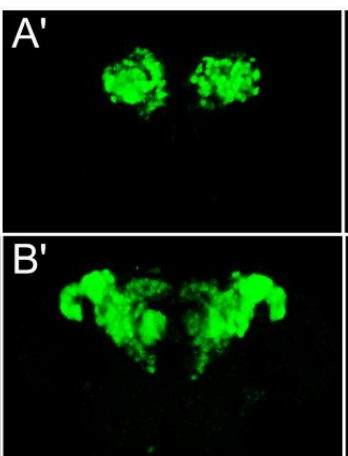

Merge

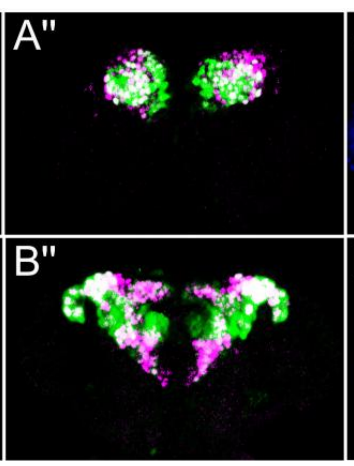

Merge/DAPI

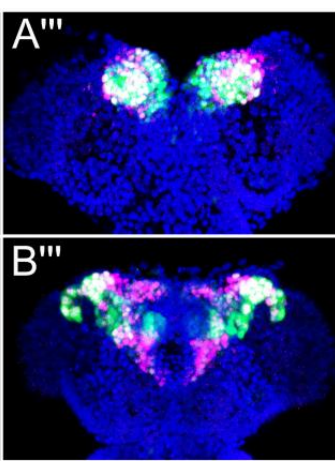

Figure 4.8 The expression of Tc-FoxQ2 and EGFP in line 86up. The expression of Tc-FoxQ2 and EGFP are largely overlapping at stage NS3 (A-A'"') and NS5 (B-B'"'). Nuclei staining with DAPI is for visualizing the morphology of the embryonic brain. Green: EGFP; magenta: Tc-FoxQ2 protein; blue: DAPI. 
To test whether the insertion was as expected in the 86up line, sequencing was performed. I wanted to test which gRNA (gRNA1 or gRNA2) worked and what the orientation of the insertion was. Two primers in the adjacent genome (AG) and one primer in EGFP were designed (Figure 4.10, yellow arrows). All the possibilities of PCR results were shown in Table 4.4. PCR results showed the distinct bands of approximately 520bp (Figure 4.9 lane1, 2: red arrowhead) and 1170bp (Figure 4.9 lane3, 4: red arrow). However, only one could be correct. The former indicated the EGFP oriented the same direction as Tc-FoxQ2 while the latter indicated the opposite direction. Other unspecific bands were not taken into consideration here. Sequencing results revealed that the band of $1170 \mathrm{bp}$ had the expected sequence while the $520 \mathrm{bp}$ band had an unrelated sequence. The repair plasmid was inserted as expected in the gRNA1 target site and EGFP on the repair plasmid was transcribed in opposite direction to the Tc-FoxQ2. The following scheme gave an overview about the repair plasmid insertion of the 86up line (Figure 4.10A). The sequences at the insertion site were shown in Figure 4.10B. To establish a homozygous line, I used genotyping based on extracting genomic DNA from wing tissue. This approach allowed dissecting wing tissue without affecting survival and fertility of adult beetles (Strobl et al., 2017).

Table 4.4 The insertion sites and the possibilities of amplificated loci by PCR.

\begin{tabular}{|l|l|c|}
\hline \multirow{2}{*}{$\begin{array}{l}\text { Insertion } \\
\text { site }\end{array}$} & \multicolumn{2}{|c|}{ Primer combination } \\
\cline { 2 - 3 } & $\begin{array}{l}\text { AG_fwd } \\
\text { EGFP_rev }\end{array}$ & $\begin{array}{l}\text { AG_rev } \\
\text { EGFP_rev }\end{array}$ \\
\hline gRNA1 & $520 \mathrm{bp}$ & $1170 \mathrm{bp}$ \\
\hline gRNA2 & $1140 \mathrm{bp}$ & $560 \mathrm{bp}$ \\
\hline
\end{tabular}

M

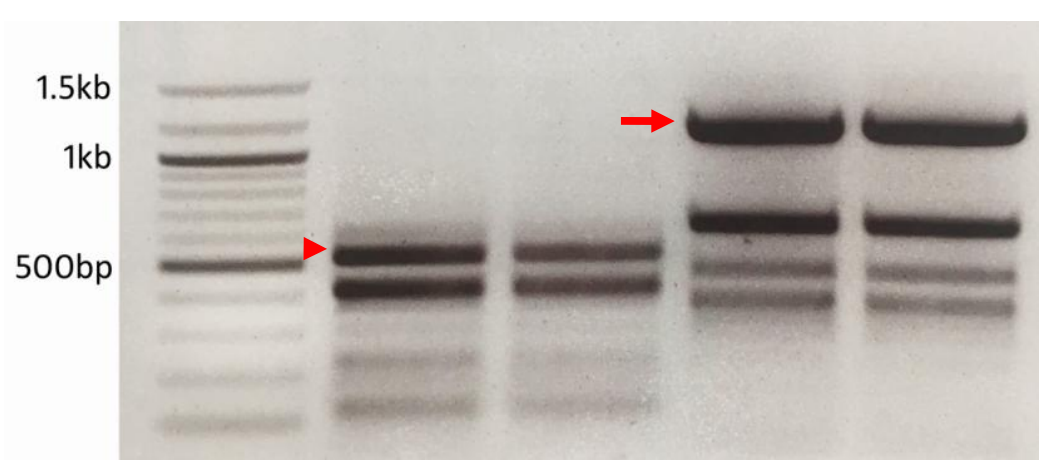

Figure 4.9 PCR analysis of the 86up line for the insertion site. Lane1 and Lane2: PCR 
product amplified with primers: AG_fwd and EGFP_rev. Lane3 and Lane4: PCR product amplified with primers: AG_rev and EGFP_rev. Red arrowhead: the potentially positive bands with the size of $520 \mathrm{bp}$. Red arrow: the potentially positive band with the size of $1170 \mathrm{bp}$. Sequencing results revealed that the band of $1170 \mathrm{bp}$ had the expected sequence.

A

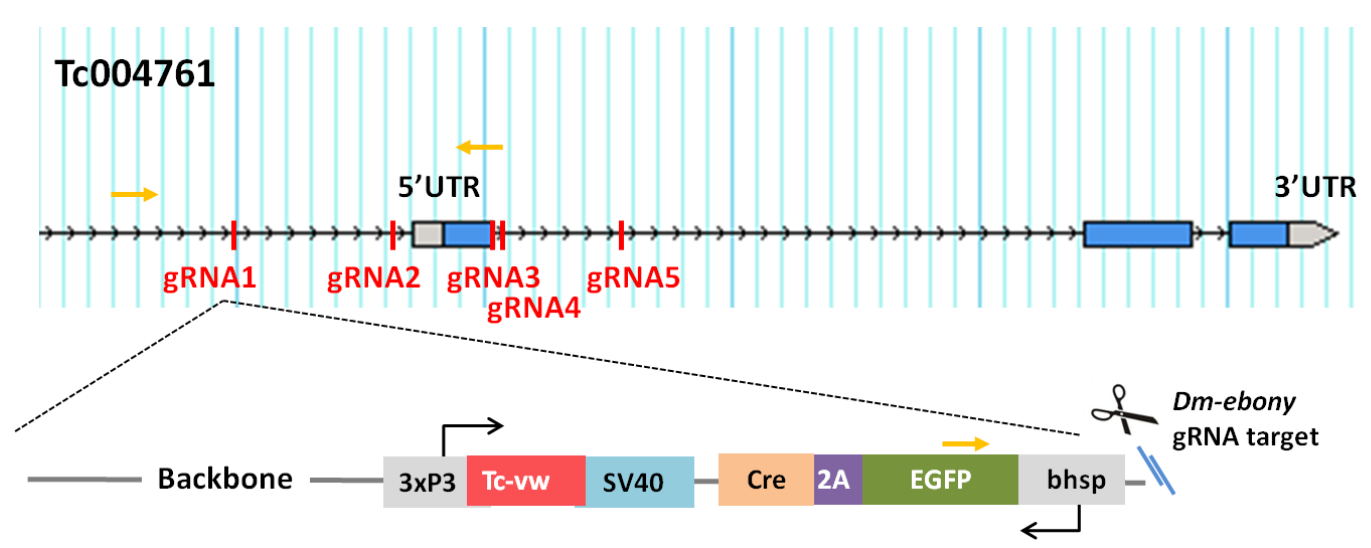

B

5'---AAACTGTATATGGGCGTTACTGTCACCCGGCGGGCTGCCCGGTTCGGGCCCGA---TGAAACGCCAGGATCCAGGAACACAA---3'

5'---TATATGGGCGTTACTGTCACCGCATCGCATATGGGCTGCCCGGTTCGGGCCCGA---TGAAACGCCATTGTGTCCAGGAACAC---3'

Figure 4.10 An overview of the insertion in genome and the alignment of the insertion. (A) The repair plasmid is inserted in gRNA1 target site and EGFP is oriented in the opposite direction to the Tc-FoxQ2. The repair plasmid is linearized at the gRNA-eb target site, which makes it bear the backbone. The yellow arrows indicate the approximately location of the primers. The space between the blue lines is $100 \mathrm{bp}$. (B) The upper row is the sequence of a perfect insertion without any indels. The lower row is the actual sequencing results of the 86up line. The PAM is highlighted in red and the gRNA target sequence is underlined. The purple bases represent the gRNA1 target sequence and the brown bases represent the gRNA-eb target sequence. The grey indicates the insertion with the size of $\sim 7 \mathrm{~kb}$. The mutations are marked in green. The CRISPR/Cas9 mediated double stranded break occurs at the DNA 3bp upstream of the PAM as predicted. Just small insertion mutations were detectable as seen in the alignment.

gRNA1 target sequence: 5' GGGCGTTACTGTCACCCTCCAGG 3'.

gRNA-eb target sequence: $5^{\prime}$ CCAGGAGGCGGGCTGCCCGGTTC 3' 


\subsection{Generation of $\mathrm{Tc}^{-F o x Q 2}{ }^{+}$neuroblasts during embryogenesis}

\subsubsection{Morphological overview of brain development in the embryo}

In Tribolium, a morphological staging system has been established with respect to early neurogenesis. This morphological staging system has 15 stages, termed NS1 to NS15 (Biffar, 2013). In this thesis, I followed this morphological staging system as a reference for the corresponding developmental stages. To visualize the overview of the morphological brain development in the embryo, nuclei were stained with DAPI. I chose those developmental stages which showed the obviously morphological changes of the brain.

Stage NS4 was the earliest stage when the brain neurogenesis occurred. At this stage, the expression of Tc-ase was first detected (data not shown), indicating NBs were delaminating from the neuroectoderm. This was consistent with what previous observations (Biffar, 2013). During stage NS3 to NS7, the brain was shown with the very thin tissue of a flat sheet of cells and NBs continuously delaminated (Figure 4.11A-C). Until stage NS7 the labral buds (Figure 4.11C: red arrow) and stomodeal buds (Figure 4.11C: red arrowhead) had formed. At stage NS11, the brain became thicker with the increasing number of the cells (Figure 4.11D). Later, the shape of the brain became oval, starting to bend towards dorsal at NS13 (Figure 4.11E). At stage NS14, the globular brain hemispheres formed and approached each other at the dorsal midline (Figure 4.11F). At stage NS15, the latest stage of the embryogenesis, the brain hemisphere approached more closely each other and increased in size and thickness, showing a pear-like shape (Figure 4.11G). I concluded that the transformation of the simple sheet-like early embryonic brain into the highly differentiated late embryonic brain involved a series of morphogenetic movements that occurred in virtually all parts of the brain. 


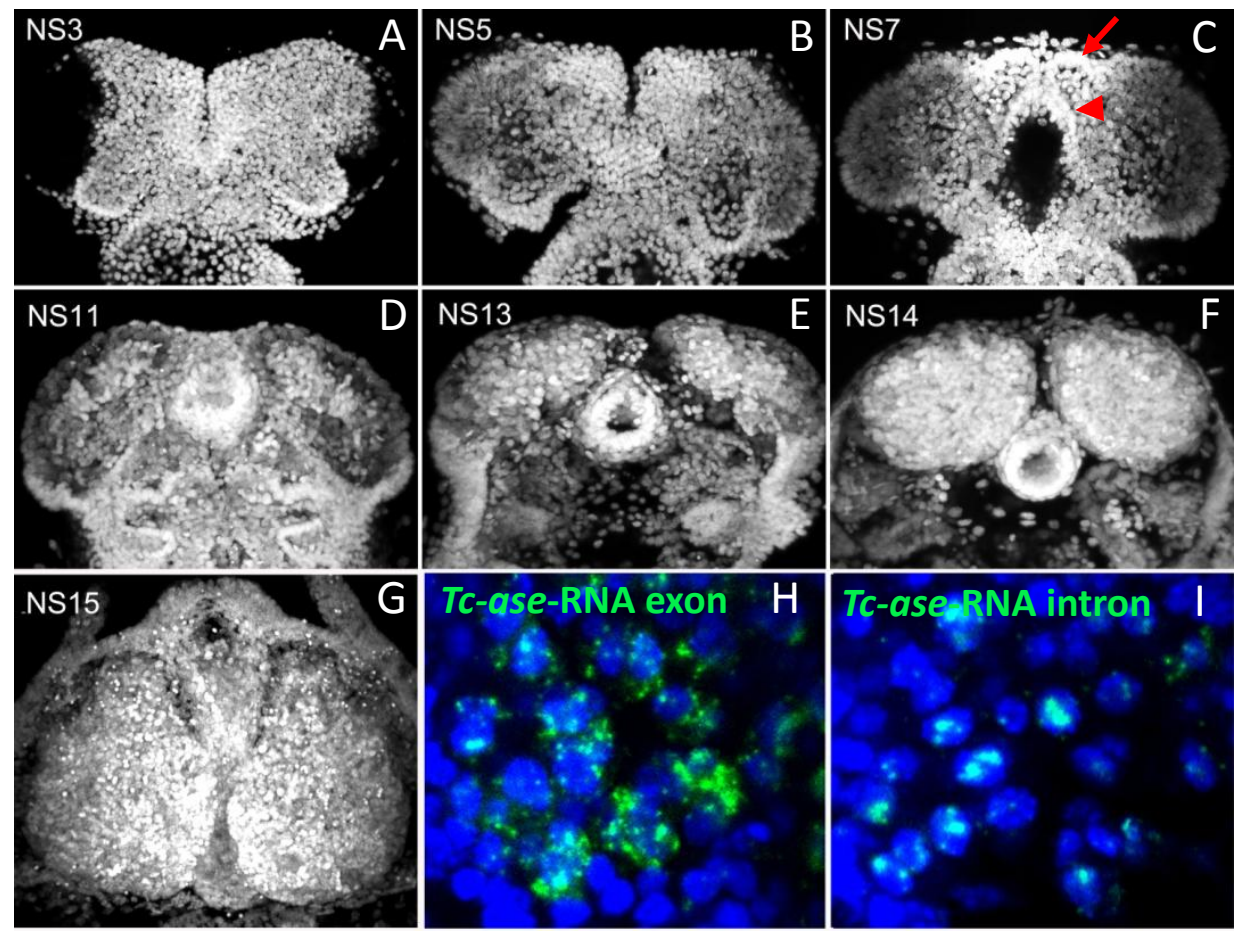

Figure 4.11 Overview of the morphological brain development from NS3 to NS15 during embryogenesis and staining of Tc-ase-RNA exonic probe and intronic probe. (A, B) At stage NS3 and NS5, the brain shows a flat sheet of cells. (C) At stage NS7, the labral and stomodeal buds have been formed (red arrow, red arrowhead). (D) At stage NS11, the brain gets thicker with the increasing number of the cells. (E, F) At stage NS13 and NS14, the shape of the brain becomes oval along with the increase in size and thickness. (G) At stage NS15, the brain hemisphere shows a pear-like shape. All planes are dorsal view. (H) The staining of $T c$-ase-RNA exonic probe, showing the marked cytoplasm. (I) The staining of Tc-ase-RNA intronic probe, showing the marked nuclei.

\subsubsection{Identification of Tc-FoxQ2+ neuroblasts in the embryonic}

\section{brain}

To identify Tc-FoxQ2 ${ }^{+} \mathrm{NBs}$, I performed Tc-FoxQ2 antibody staining and Tc-ase in situ hybridization. The Tc-ase-RNA exonic probe marked the cytoplasm, which made it difficult to see the border between cells (Figure $4.11 \mathrm{H}$ ). I therefore used a Tc-ase-RNA intronic probe, which marked only nuclei, in order to get better resolution (Figure 4.11I). In addition, NBs could be morphologically distinguished by the large size of their nuclei, their round shape and position (below the peripheral neuroectoderm).

The earliest delaminated NBs were detected at stage NS4. However, I did not detect any NBs expressing Tc-FoxQ2 (data not shown). At stage NS8, the number of 
the NBs increased, showing that more NBs had delaminated. At that stage, 15 Tc-FoxQ2 ${ }^{+}$NBs per hemisphere were observed and divided into two groups: a large group of $\sim 12$ FoxQ $^{+} \mathrm{NBs}$ in the anterior ( $n=6$; Figure 4.12A": blue, green and orange) and a smaller group of $3 \mathrm{Tc}-\mathrm{FoxQ}^{+} \mathrm{NBs}$ close to the basis of the labrum ( $n=6$; Figure 4.12A": grey). By stage NS11, the number of the Tc-FoxQ2 ${ }^{+} \mathrm{NBs}$ in the anterior group decreased to 8 to 11 ( $n=6$; Figure 4.12B": blue, green and orange) and only one Tc-FoxQ2 ${ }^{+} \mathrm{NBs}$ was detected at the posterior of the labrum ( $n=6$; Figure 4.12B": grey). At the late stage NS14, there were just 5 to 7 Tc-FoxQ2 ${ }^{+} \mathrm{NBs}$ that were scattered and had incompact arrangement in the anterior region $(n=6$; Figure $4.12 C^{\prime \prime}$ : blue and orange). At stage NS15, the dynamic morphogenetic movement of the brain and much more cells made it difficult to identify the Tc-FoxQ2 $^{+}$NBs. The raw counts of the Tc-FoxQ2 ${ }^{+}$NBs were displayed in Table 7.4. Based on this molecular marker analysis, I identified $\sim 15 \mathrm{Tc}^{-}$-FoxQ2 ${ }^{+} \mathrm{NBs}$ at stage NS8, 9 to 12 Tc-FoxQ2 ${ }^{+}$NBs at stage NS11 and 5 to 7 Tc-FoxQ2 ${ }^{+}$NBs at stage NS14. I concluded that the number of FoxQ2 ${ }^{+} \mathrm{NBs}$ decreased during embryogenesis.

In Drosophila, the brain is divided into the protocerebrum (PC), deutocerebrum (DC) and tritocerebrum (TC). And within the protocerebrum, NBs are subdivide into an anterior $(\mathrm{Pa})$, central $(\mathrm{Pc})$ and posterior $(\mathrm{Pp})$ group (Younossi-Hartenstein et al., 1996). By using this as a reference, I could say that both groups of Tc-FoxQ2 ${ }^{+}$ NBs were in protocerebrum anterior $(\mathrm{Pa})$ region. From stage NS8 to NS11, the spatial arrangement of Tc-FoxQ2 ${ }^{+} \mathrm{NBs}$ did not change much except for the number. Two Tc-FoxQ2 ${ }^{+} \mathrm{NBs}$ were able to be followed over time (Figure 4.12A", B": green and orange) due to their position relatively distant from other FoxQ2 ${ }^{+} \mathrm{NBs}$ that were arranged closely in the anterior group. In order to easily describe in the following, I named the two Tc-FoxQ2 ${ }^{+} \mathrm{NBs}$ anterior-posteroventral (A-PV) (Figure 4.12A", B": green) and anterior-posterodorsal (A-PD) (Figure 4.12A", B": orange), respectively. The A-PD Tc-FoxQ2 ${ }^{+} \mathrm{NB}$ moved toward to anterior-dorsal region, while the A-PV FoxQ2 ${ }^{+} \mathrm{NB}$ approached other anterior Tc-FoxQ2 ${ }^{+} \mathrm{NBs}$ from stage NS8 to NS11. At stage NS14 when the brain morphology had changed much, the A-PD Tc-FoxQ2 ${ }^{+} \mathrm{NB}$ could be still identified due to its position (Figure $4.12 \mathrm{C}^{\prime \prime}$ : orange). However, it was difficult to determine the A-PV Tc-FoxQ2 ${ }^{+} \mathrm{NB}$ because of the declined number of Tc-FoxQ2 ${ }^{+} \mathrm{NBs}$ and the altered spatial arrangement (Figure 4.12C": blue). In addition, two Tc-FoxQ2 ${ }^{+} \mathrm{NBs}$ which derived from the anterior group were located close to the midline at stage NS14, thus I called them Tc-FoxQ2 anteromedian (FAM) NBs: FAM1 NB and FAM2 NB, respectively (Figure 4.12C": red circle). 

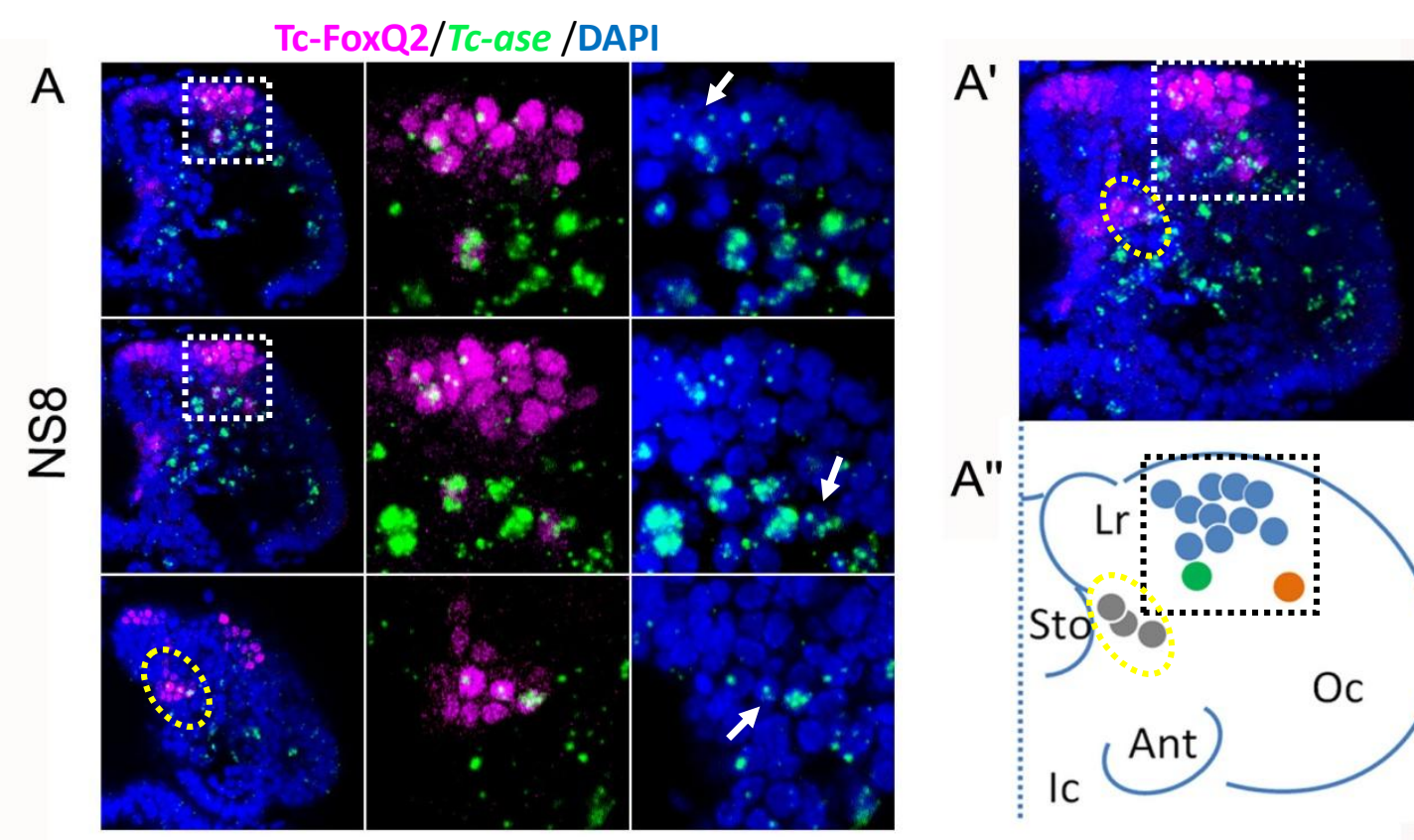

A"

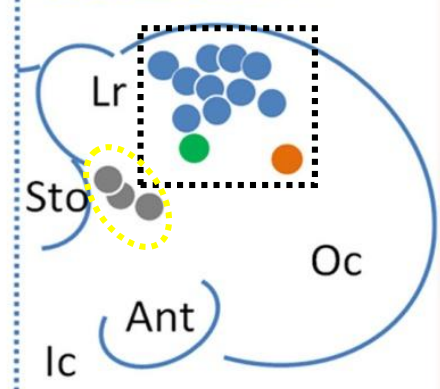

B

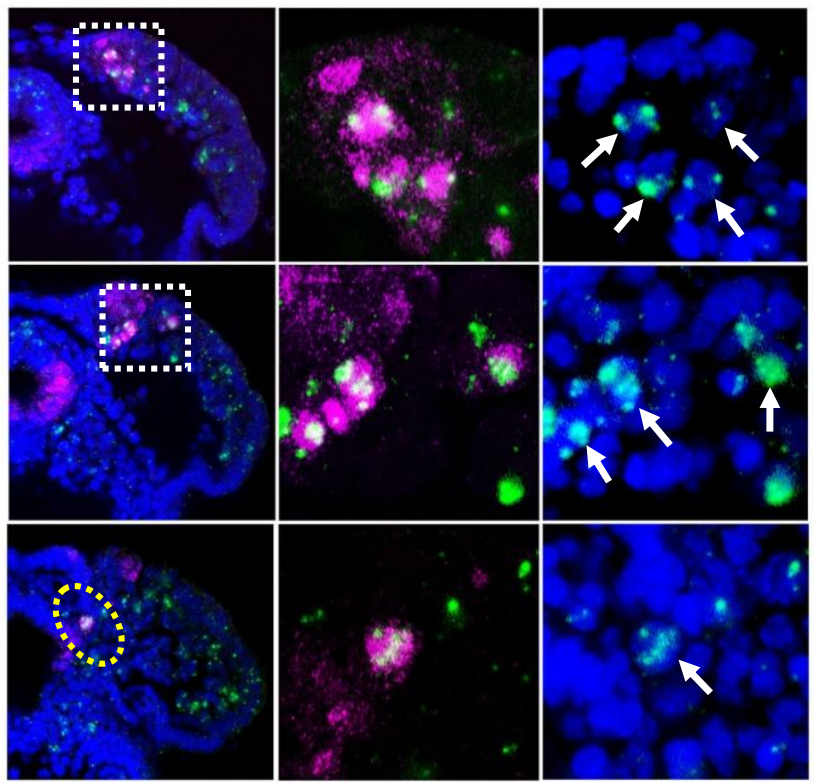

$B^{\prime}$

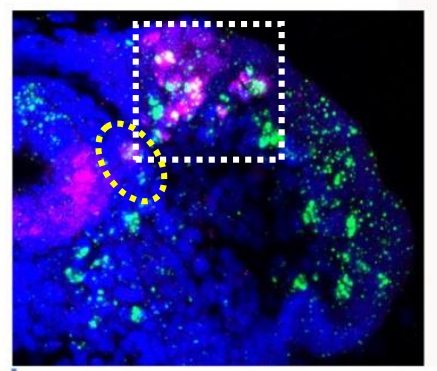

$B^{\prime \prime}$

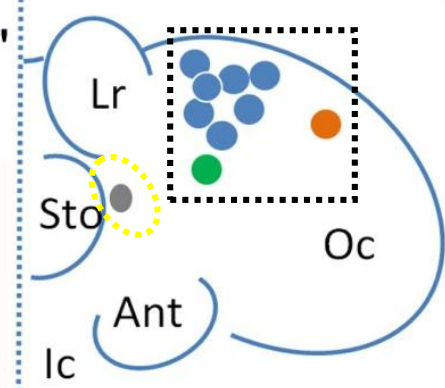

C

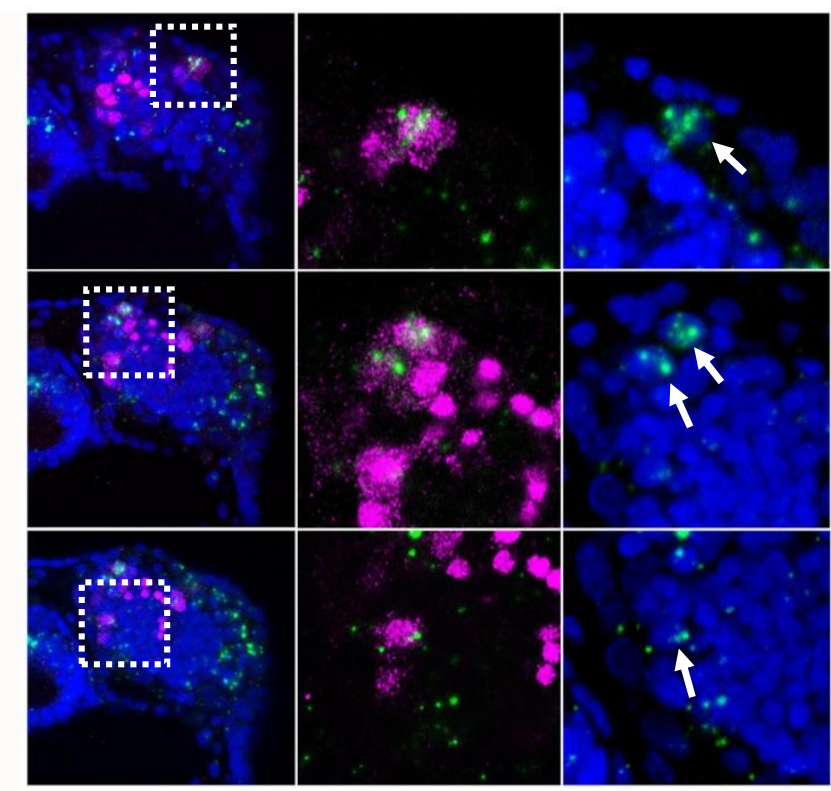

$C^{\prime}$

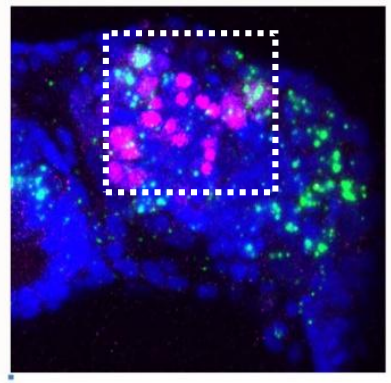

$C^{\prime \prime}$

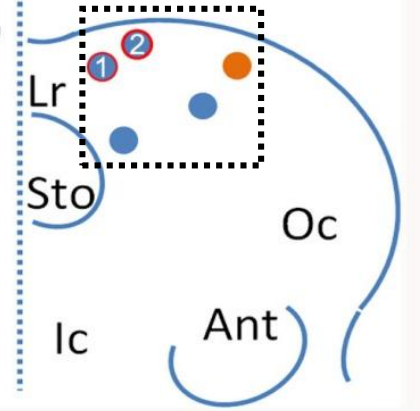


Figure 4.12 Tc-FoxQ2 ${ }^{+}$NBs pattern during embryogenesis. (A-C) Each stage shows multiple focal planes from dorsal to ventral views of the hemisphere to clearly visualize each Tc-FoxQ2 ${ }^{+}$NBs. (A) 15 Tc-FoxQ2 ${ }^{+}$NBs are identified at stage NS8. (B) $\sim 10$ Tc-FoxQ2 ${ }^{+}$ $\mathrm{NBs}$ are identified at stage NS11. (C) $\sim 5$ Tc-FoxQ2 ${ }^{+} \mathrm{NBs}$ are identified at stage NS14. Arrows indicate the identified TC-FoxQ2 ${ }^{+} \mathrm{NBs}$. $\left(\mathrm{A}^{\prime}-\mathrm{C}^{\prime}\right)$ Overview of the all planes of the brain hemisphere. Magenta: Tc-FoxQ2 antibody; green: Tc-ase-RNA intronic probe; blue: DAPI. $\left(A^{\prime \prime}-C^{\prime \prime}\right)$ Summary of TC-FoxQ2 ${ }^{+} \mathrm{NBs}$ formation. Blue: the anterior Tc-FoxQ2 ${ }^{+} \mathrm{NBs}$; green: the A-PV Tc-FoxQ2 ${ }^{+} \mathrm{NB}$; orange: the A-PD Tc-FoxQ2 ${ }^{+} \mathrm{NB}$; grey: the Tc-FoxQ2 ${ }^{+} \mathrm{NBs}$ at the basis of the labrum; red circle: the FAM1 NB and FAM2 NB. Lr: labrum; Sto: stomodeum; Oc: ocular; Ic: intercalary; Ant: antenna. The dashed box (black or white) represents the anterior region of the brain hemisphere where most Tc-FoxQ2 ${ }^{+} \mathrm{NBs}$ are located. The dashed circle (yellow) represents the basal domain of the labrum.

\subsubsection{Candidate genes for Tc-FoxQ2+ neuroblasts identity specification}

In insects, the head epidermis and the brain derive from the same early neuroectodermal precursor cells. Hence, the genes identified to pattern the head are most likely also involved in brain patterning. The identity of the NB is specified by the combination of several regulators (Bossing et al., 1996; Schmid et al., 1999; Urbach and Technau, 2003). The genes that probably specify NB identity are critical for describing the characteristics of each NB.

Therefore, I wondered what transcription factors possibly specified the Tc-FoxQ $2^{+}$ NBs. Here I selected candidate genes which were known to be expressed in anterior head or even to play an important role in head development (Posnien et al., 2011b; Kitzmann et al., 2017). Of those, I tested the genes that showed co-expression with Tc-FoxQ2 in neurogenic region. I performed Tc-FoxQ2 antibody staining in combination with in situ hybridization of individual transcription factors and nuclei marker DAPI in the embryos $(n=6)$. From stage NS14 onwards, I was unable to test the Tc-FoxQ2 ${ }^{+} \mathrm{NBs}$ for expression with other transcription factors. During late stages of the embryonic development, the dynamic morphogenetic movement of the brain and the large number of differentiating cells made it more difficult to determine and identify individual cells with in situ hybridization. I therefore restricted the analysis of Tc-FoxQ2 ${ }^{+} \mathrm{NBs}$ with other transcription factors to the certain stages (NS8-NS11) of embryogenesis.

Tc-six3, a transcription factor which belongs to sine oculis homeobox transcription factor family, is a major regulator of the anterior median head and known to be 
required for the embryonic formation of the central complex in Tribolium (Posnien et al., 2011b). The ortholog in Drosophila is optix which has functions in eye development. Ectopic expression of optix results in ectopic eyes formation (Seimiya and Gehring, 2000). In Tribolium, a previous study showed that the expression of $T c$-foxQ2 and $T c$-six 3 were largely overlapping in the anterior median region from early stages on and they were co-expressed in the labrum and the anterior portion of the protocerebrum at later stages (Kitzmann et al., 2017). At stage NS8, the earliest stage when Tc-FoxQ2 ${ }^{+} \mathrm{NBs}$ had delaminated, Tc-six3 mRNA was detected to cover the complete Tc-FoxQ2 expression domains, indicating Tc-six3 was expressed in all FoxQ2 ${ }^{+}$NBs (Figure 4.13A, A'). At later stage NS11, though the location of the A-PD Tc-FoxQ2 ${ }^{+}$NB had changed a bit, moving toward to anterior-dorsal side, $T c$-six 3 expression domain remained constant and was detectable in all Tc-FoxQ2 ${ }^{+}$NBs (Figure 4.14A, $A^{\prime}$ ). Therefore, from stage NS8 to NS11, all Tc-FoxQ2 ${ }^{+}$NBs were Tc-six3 positive.

Tc-six4 is a Six family homeodomain transcription factor. It has been identified as a marker for the insect head placode and also gives rise to neural cells (Posnien et al., 2011a). In Drosophila, six4 is required for the proper development of the cells deriving from the mesoderm, including fat body, gonad and muscles (Clark et al., 2006). In Tribolium, there was no overlap of Tc-six4 and Tc-foxQ2 at early stages. Co-expression started from mid embryonic stage when Tc-six4 was partially overlapping with Tc-foxQ2 expression domain within the neurogenic region (Kitzmann et al., 2017). At stage NS8, Tc-six4 expression almost completely covered the Tc-FoxQ2 expression region and was detected in all Tc-FoxQ2 ${ }^{+} \mathrm{NBs}$ except the A-PD Tc-FoxQ2 ${ }^{+}$NB (Figure 4.13B, B'). At stage NS11, the TC-six4 expression domain had not changed, showing the expression in Tc-FoxQ2 ${ }^{+} \mathrm{NBs}$ except for the A-PD Tc-FoxQ2 ${ }^{+}$NB (Figure 4.14B, B').

Tc-chx is a transcription factor that is expressed in the anterior median head and marks the pars intercerebralis (PI) which is a part of neuroendocrine system (Posnien et al., 2011b; Kitzmann et al., 2017). In Tribolium it has been proven that Tc-chx is involved in the central body formation (Koniszewski, 2011). Two homologues $V s \times 1$ and $V s \times 2$ in Drosophlia are identified, which are required for optic lobe development (Erclik et al., 2008). Further, in Drosophlia Vsx is also shown to be expressed in the $\mathrm{PI}$, the region where the $\mathrm{CX}$ derives from (Boyan and Williams, 1997; De Velassco et al., 2007). In Tribolium, at early stages there was a little overlap of $T c$-chx and Tc-foxQ2 in the posterior portion of the labrum. Later, an additional co-expression appeared in the neurogenic region (Kitzmann et al., 2017). At stage NS8, the expression of Tc-chx showed no overlap with Tc-FoxQ2 and seemed mutually exclusive (Figure 4.13C, $C^{\prime}$ ). I did not observe any Tc-FoxQ2 ${ }^{+}$ NB expressing Tc-chx. However, during the course of development, Tc-chx expression domain was dynamic and additional expression emerged, which led to co-expression with Tc-FoxQ2. Apart from the A-PD Tc-FoxQ2 ${ }^{+} \mathrm{NB}$, all Tc-FoxQ2 ${ }^{+} \mathrm{NBs}$ were $T c$-chx positive at stage NS11 (Figure 4.14C, $C^{\prime}$ ). Noticeably there was only 
weak expression of Tc-chx in Tc-FoxQ2 ${ }^{+}$NBs.

Tc-retinal homeobox (Tc- $r x$ ) which encodes for a transcription factor containing a paired-like homeodomain plays a role in labrum formation and neuroectodermal development in Tribolium (Koniszewski, 2011; Posnien et al., 2011b). The $r x$ gene is well conserved from fly to human (Mathers et al., 1997, Eggert et al., 1998; Walldorf et al., 2000, D'Aniello et al., 2011). In vertebrates, $R x$ is essential for brain and eye development (Mathers et al., 1997; Bailey et al., 2004). The homologue of $r x$ in Drosophila is required for brain and clypeus development but not essential for eye formation (Davis et al., 2003). Recently the studies have shown that $r x$ is expressed in the MB NBs and controls the cell proliferation and growth of the MB NBs, and is required for the MB development (Kunz et al., 2012; Kraft et al., 2016). In Tribolium, the expression of Tc-rx and Tc-foxQ2 was little overlapping in the neuroectoderm at early stages and then additional co-expression in the labrum appeared (Kitzmann et al., 2017). From stage NS8 to NS11, Tc-rx expression was detected only in the A-PD Tc-FoxQ2 ${ }^{+}$NB (Figure 4.13D, $D^{\prime}$; Figure 4.14D, D').

Tc-eyeless (Tc-ey) is a transcription factor that is involved in anterior median head patterning in Tribolium (Posnien et al., 2011b). It also shows the expression in anteriormost tissue, which contributes to the putative mushroom body (MB) (Posnien et al., 2011b). In Drosophila, ey is known to be expressed in the MB NBs in the embryo and involved in the appropriate development of the MBs (Kurusu et al., 2000, Noveen et al., 2000; Kunz et al., 2012). In Tribolium, the expression of Tc-ey started in a prominent ocular region and then an additional anterior median expression arised (Posnien et al., 2011b). At stage NS8 only A-PD Tc-FoxQ2 ${ }^{+}$NB was observed to express Tc-ey (Figure 4.13E, E'). At stage NS11, in addition to A-PD Tc-FoxQ2 ${ }^{+} \mathrm{NB}, \mathrm{Tc}$-ey was also detected in the A-PV Tc-FoxQ2 ${ }^{+} \mathrm{NB}$ (Figure 4.14E, E').

Fez is a zinc-finger gene which encodes a transcriptional repressor (Hashimoto et al., 2000; Hirata et al., 2006). It has been reported that Tc-fez1 is involved in insect head epidermis patterning (Posnien et al., 2011b). Tc-fez1 was expressed in ocular and small region of the anterior neuroectoderm (Posnien et al., 2011b). Tc-fez1 was detected in A-PV and A-PD Tc-FoxQ2 ${ }^{+}$NBs at stage NS8 (Figure 4.13F, $\mathrm{F}^{\prime}$ ). Later, the expression domain of $T c-f e z 1$ expanded. In addition to A-PV and A-PD Tc-FoxQ2 ${ }^{+}$NBs, one more FoxQ2 ${ }^{+}$NB was observed to express Tc-fez1 (Figure 4.14F, $\left.F^{\prime}\right)$.

Tc-scarecrow (Tc-scro/nk2.1) is a transcription factor of homeobox family with roles in labrum and stomodeum development in Tribolium (Kittelmann, 2012; Kittelmann et al., 2013; Posnien et al., 2011b). The homologue in Drosophila is a member of NK-2 homeobox gene family, which is expressed in the procephalic NBs that generate the neurons in the brain (Zaffran et al., 2000). In Tribolium, the co-expression of Tc-scro and Tc-foxQ2 showed in the lateral portion 
of labrum, the stomodeum and additionally small region of the anterior neuroectoderm (Kitzmann et al., 2017). At stage NS8, Tc-scro expression was observed only in the A-PD Tc-FoxQ2 ${ }^{+}$NB (Figure 4.13G, G'). With development, the original TC-scro expression domain in neurogenic region moved towards to the anterior, which skipped the A-PD Tc-FoxQ2 ${ }^{+} \mathrm{NB}$. And an additionally small Tc-scro expression domain appeared, covering the A-PV Tc-FoxQ2 ${ }^{+} \mathrm{NB}$ and the neighbor $\mathrm{Tc}^{-F o x Q 2}{ }^{+} \mathrm{NB}$ (Figure 4.14G, G'). Furthermore, Tc-scro expression was always detected in the Tc-FoxQ2 ${ }^{+} \mathrm{NBs}$ in the basal domain of the labrum (Figure 4.13G", $\mathrm{G}^{\prime \prime \prime}$ : dashed yellow circle; Figure 4.14G"', G'"': dashed yellow circle).

In summary, at stage NS8, all Tc-FoxQ2 ${ }^{+} \mathrm{NBs}$ were $\mathrm{Tc}$-six3 positive (Figure $4.13 \mathrm{~A}, \mathrm{~A}^{\prime}$ ). Tc-six4 expression was detected in all Tc-FoxQ2 ${ }^{+} \mathrm{NBs}$ except the A-PD Tc-FoxQ2 ${ }^{+} \mathrm{NB}$ (Figure 4.13B, $\mathrm{B}^{\prime}$ ). By contrast, the expression of $T c-r x$ and $T c-e y$ were detected only in the A-PD Tc-FoxQ2 ${ }^{+} \mathrm{NB}$, and $T c-f e z 1$ was detected in the A-PV and A-PD Tc-FoxQ2 ${ }^{+} \mathrm{NBs}$ (Figure 4.13D, D', E, E', F, F'). However, I did not observe any $\mathrm{TC}^{-}$FoxQ2 ${ }^{+} \mathrm{NBs}$ expressing Tc-chx at this stage (Figure 4.13C, $\mathrm{C}^{\prime}$ ). Furthermore, Tc-scro expression was observed in the A-PD Tc-FoxQ2 ${ }^{+} \mathrm{NB}$ and the Tc-FoxQ2 ${ }^{+} \mathrm{NBs}$ in the basal domain of the labrum (Figure 4.13G-G'"'). At the later stage NS11, Tc-six3, Tc-six4 and Tc-rx expression domain remained constant while Tc-chx, Tc-ey, $T c-f e z 1$ and Tc-scro showed the dynamic expression pattern. Though the number of the Tc-FoxQ2 ${ }^{+}$NBs was less at stage NS11 than that of stage NS8, all of them were Tc-six3 positive (Figure 4.14A, $A^{\prime}$ ). And all the Tc-FoxQ2 ${ }^{+} \mathrm{NBs}$ except the A-PD Tc-FoxQ2 ${ }^{+}$NBs remained Tc-six4 positive (Figure 4.14B, $\mathrm{B}^{\prime}$ ). Tc-rx was still detected only in the A-PD TC-FoxQ2 ${ }^{+} \mathrm{NB}$ (Figure 4.14D, D'). Compared to stage NS8, transcription factors Tc-chx, Tc-ey, Tc-fez1 and Tc-scro were expressed in more Tc-FoxQ2 ${ }^{+}$NBs at stage NS11. None of the Tc-FoxQ2 ${ }^{+}$NBs were Tc-chx positive at NS8 (Figure 4.13C, $C^{\prime}$ ), whereas only the A-PD Tc-FoxQ2 ${ }^{+}$NB was not Tc-chx positive and the weak $T c$-chx expression was detectable in other Tc-FoxQ2 ${ }^{+} \mathrm{NBs}$ at NS11 (Figure 4.14C, $C^{\prime}$ ). Tc-ey was not only expressed in the A-PD TC-FoxQ2 ${ }^{+}$NBs but also in the A-PV Tc-FoxQ2 ${ }^{+} \mathrm{NB}$ (Figure 4.14E, $\mathrm{E}^{\prime}$ ). In addition to the A-PD and A-PD Tc-FoxQ2 ${ }^{+} \mathrm{NBs}$, one more Tc-FoxQ2 ${ }^{+} \mathrm{NB}$ was observed to express $T c-f e z 1$ (Figure 4.14F, $\mathrm{F}^{\prime}$ ). I did not observe the A-PD Tc-FoxQ2 ${ }^{+} \mathrm{NB}$ expressing Tc-scro anymore but Tc-scro expression was detected in the A-PV Tc-FoxQ2 ${ }^{+} \mathrm{NB}$ and its neighbor Tc-FoxQ2 ${ }^{+} \mathrm{NB}$ as well as the $\mathrm{TC}_{-} \mathrm{FoxQ}_{2}{ }^{+} \mathrm{NBs}$ in the basal domain of the labrum (Figure 4.14G-G'"'). In addition, the expression of other transcription factors was not detectable in the Tc-FoxQ2 ${ }^{+} \mathrm{NBs}$ in the basal domain of the labrum except Tc-scro.

Taken together, the number of the Tc-FoxQ2 ${ }^{+} \mathrm{NBs}$ decreased during embryogenesis. Tc-FoxQ2 ${ }^{+}$NBs were specified by a unique combination of the transcription factors. The later stage the more transcription factors were observed to express in the Tc-FoxQ2 ${ }^{+}$NBs. 
NS8 Tc-FoxQ2/TFs/DAPI
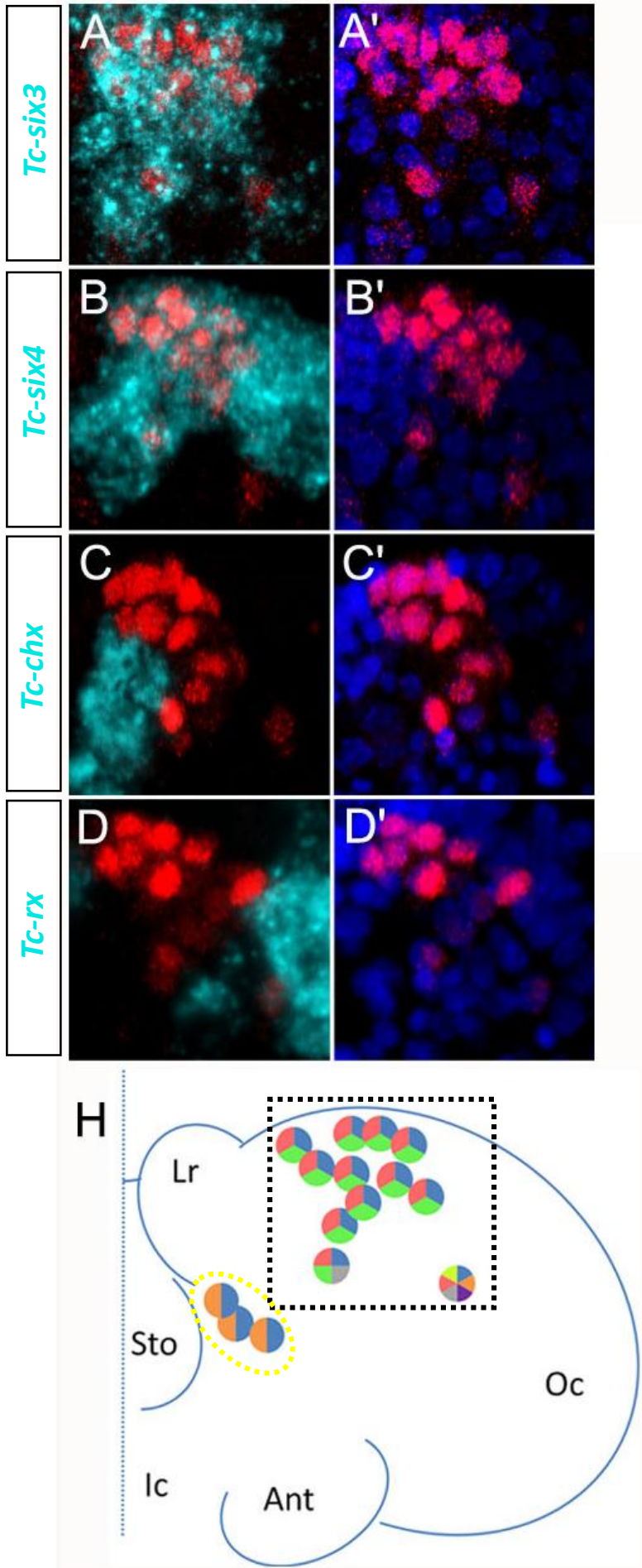

Tc-FoxQ2/TFs/DAPI
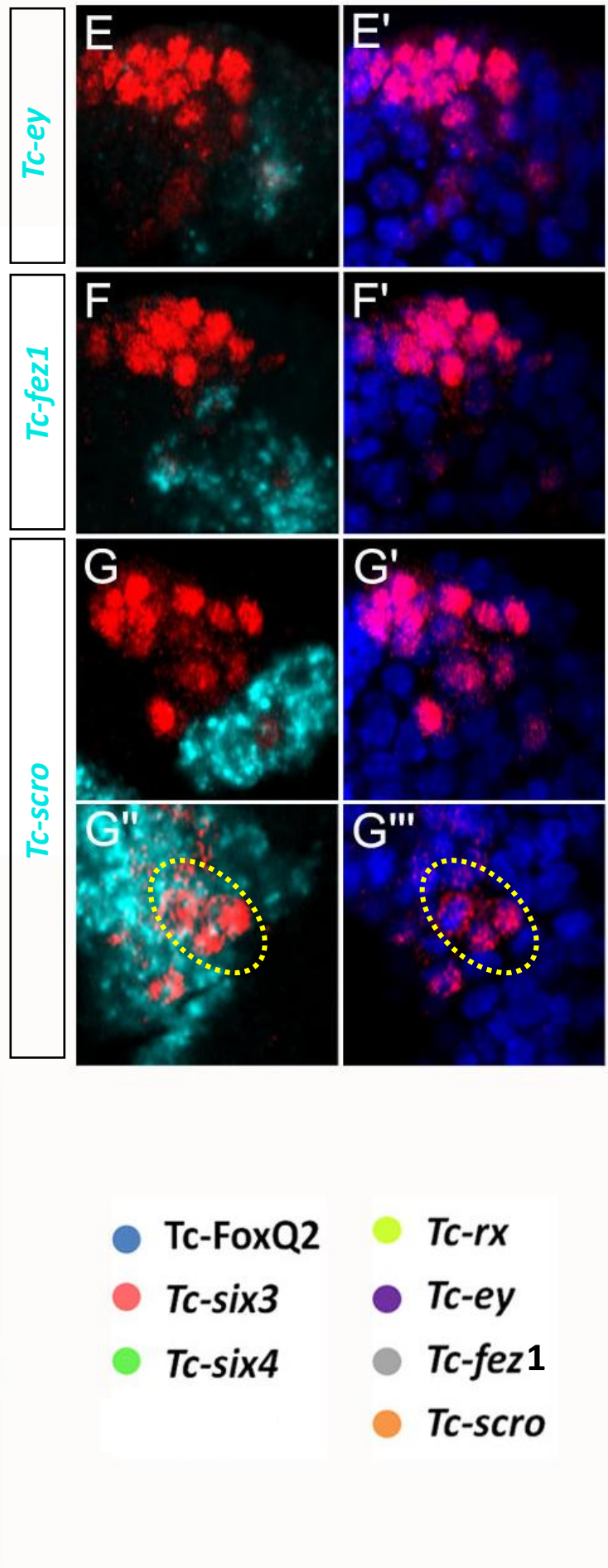

Figure 4.13 Tc-FoxQ2 ${ }^{+}$NBs express transcription factors at stage NS8. (A) Co-expression of $T c$-six3 and Tc-FoxQ2. All Tc-FoxQ2 ${ }^{+} \mathrm{NBs}$ are $T c$-six3 positive. (B) Co-expression of Tc-six4 and Tc-FoxQ2. Tc-six4 is not expressed in the A-PD Tc-FoxQ2 ${ }^{+}$NB. (C) Tc-chx and Tc-FoxQ2 shows mutually exclusive expression. (D) Co-expression of $T c-r x$ and Tc-FoxQ2. Only the A-PD Tc-FoxQ2 ${ }^{+}$NB is Tc-rx positive. (E) Co-expression of Tc-ey and Tc-FoxQ2. Only the A-PD Tc-FoxQ2 ${ }^{+}$NB shows the expression of Tc-ey. (F) Co-expression of Tc-fez1 and Tc-FoxQ2. 
The A-PV and A-PD Tc-FoxQ2 $2^{+}$NBs are Tc-fez1 positive. (G, G') Co-expression of Tc-srco and Tc-FoxQ2. The A-PD Tc-FoxQ2 ${ }^{+} \mathrm{NB}$ and the Tc-FoxQ2 ${ }^{+} \mathrm{NBs}$ in basal domain of the labrum are Tc-srco positive. ( $\left.A^{\prime}-G^{\prime}, G^{\prime \prime \prime}\right)$ DAPI staining visualizes the Tc-FoxQ2 expression in the nuclei. Red: Tc-FoxQ2 antibody; cyan: the transcription factors; blue: DAPI. (H) Summary of the transcription factors expression in the Tc-FoxQ2 ${ }^{+} \mathrm{NBs}$ at stage NS8. The dashed box (black) represents the anterior region (A-G) and $\left(A^{\prime}-G^{\prime}\right)$. The dashed circle (yellow) represents the basal domain of the labrum (G", $\left.G^{\prime \prime \prime}\right)$.

NS11 Tc-FoxQ2/TFs/DAPI
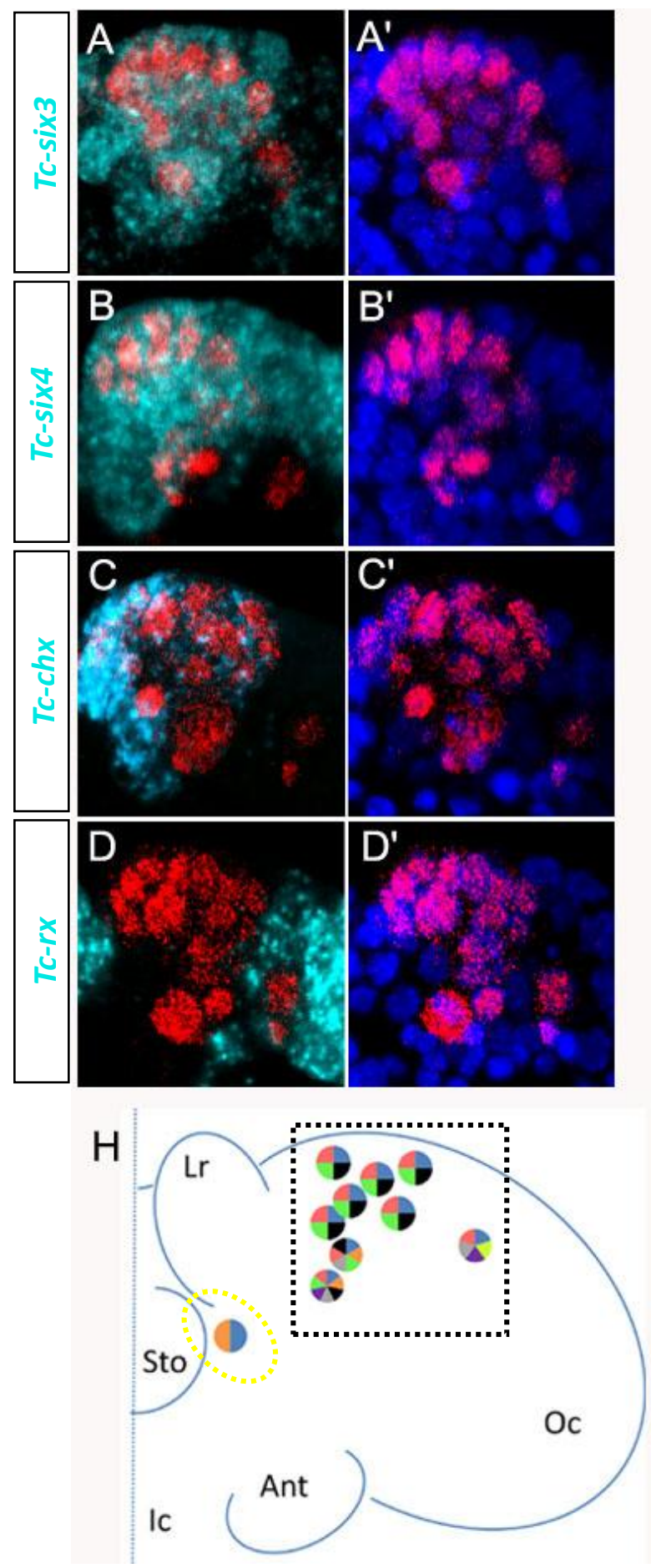

Tc-FoxQ2/TFs/DAPI

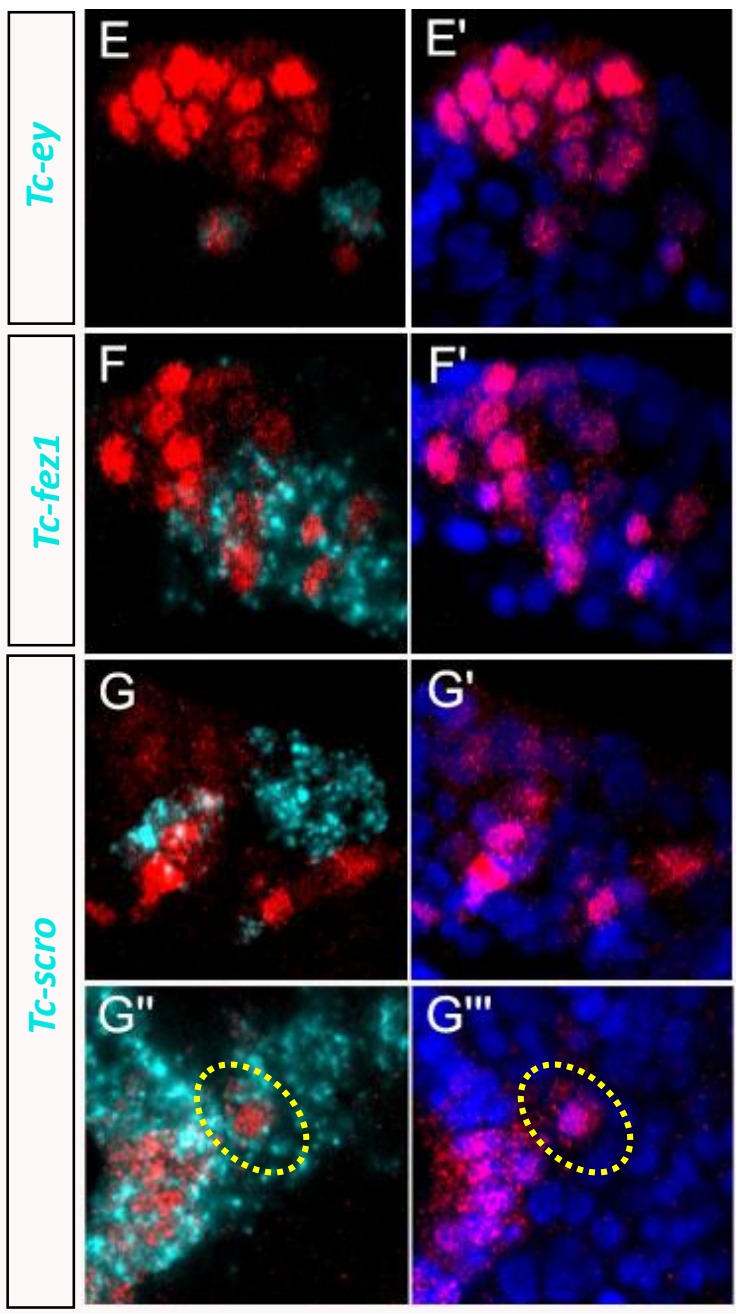

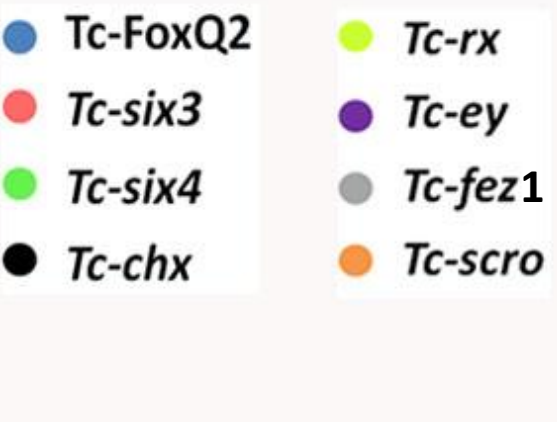


Figure 4.14 Tc-FoxQ2 ${ }^{+}$NBs express transcription factors at stage NS11. (A) Co-expression of $T c$-six3 and Tc-FoxQ2. All Tc-FoxQ2 ${ }^{+} \mathrm{NBs}$ show the expression of $T c$-six3. (B) Co-expression of Tc-six4 and Tc-FoxQ2. Tc-six4 is not expressed in the A-PD Tc-FoxQ2 ${ }^{+} \mathrm{NB}$. (C) Co-expression of Tc-chx and Tc-FoxQ2. The A-PD Tc-FoxQ2 ${ }^{+} \mathrm{NB}$ is Tc-chx negative while other Tc-FoxQ2 ${ }^{+}$NBs show weak Tc-chx expression. (D) Co-expression of Tc-rx and Tc-FoxQ2. Only the A-PD Tc-FoxQ2 ${ }^{+}$NB shows Tc-rx expression. (E) Co-expression of Tc-ey and Tc-FoxQ2. The A-PV and A-PD Tc-FoxQ2 ${ }^{+} \mathrm{NB}$ are Tc-ey positive. (F) Co-expression of Tc-fez1 and Tc-FoxQ2. In addition to the A-PV and A-PD Tc-FoxQ2 ${ }^{+} \mathrm{NBs}$, one more FoxQ2 ${ }^{+} \mathrm{NB}$ shows expression of Tc-fez1. (G, G') Co-expression of Tc-srco and Tc-FoxQ2. The A-PV $\mathrm{TC}_{-}-\mathrm{FoxQ2} 2^{+} \mathrm{NB}$ and the neighbor Tc-FoxQ2 ${ }^{+} \mathrm{NB}$ show the expression of $\mathrm{TC}$-srco, and the Tc-FoxQ2 ${ }^{+} \mathrm{NBs}$ in basal domain of the labrum remain Tc-srco positive. ( $\left.A^{\prime}-G^{\prime}, G^{\prime \prime \prime}\right)$ DAPI staining visualizes the Tc-FoxQ2 expression in the nuclei. Red: Tc-FoxQ2 antibody; cyan: the transcription factors; blue: DAPI. (H) Summary of the transcription factors expression in the Tc-FoxQ2 ${ }^{+}$NBs at stage NS11. The dashed box (black) represents the anterior region $(A-G),\left(A^{\prime}-G^{\prime}\right)$ and $\left(G^{\prime \prime}, G^{\prime \prime \prime}\right)$. The dashed circle (yellow) represents the basal domain of the labrum $\left(G^{\prime \prime}, G^{\prime \prime \prime}\right)$.

\subsection{Description of the $T c-f o x Q 2$ enhancer trap line}

\subsubsection{Co-expression analysis of Tc-FoxQ2 and EGFP in the embryo}

To investigate in how far the expression of Tc-FoxQ2 and EGFP of the 86up line overlapped, antibody staining was performed in the embryo, larva and adult. The co-expression of Tc-FoxQ2 and EGFP were analyzed from early stage (NS3) to the latest stage of embryogenesis (NS15).

TC-FoxQ2 and EGFP expression were almost completely overlapping from early stages onwards. At early stage NS3, Tc-FoxQ2 expression showed two round domains within the non-neural anterior median region (Figure 4.15A- $A^{\prime \prime \prime}$ ). The expression of EGFP was almost completely overlapping with Tc-FoxQ2. Only a few Tc-FoxQ2 positive cells in the anterior most outside were not expressing EGFP (Figure 4.15A-A", arrowheads). A narrow stripe of cells which were located close to the midline showed weaker Tc-FoxQ2 signal than most other cells, whereas EGFP expression in these cells was strong. This revealed that all EGFP positive cells were expressing Tc-FoxQ2, whereas not all Tc-FoxQ2 positive cells were also positive for the EGFP. In other words, EGFP marked the subset of Tc-FoxQ2 positive cells.

At stage NS6, the expression of Tc-FoxQ2 was splitted into several domains. Tc-FoxQ2 expression was detected in the labrum, stomodeum as well as the anterior neuroectodermal region, exhibiting a 'goat head' shape pattern (Figure 4.15B-B'"'). At the same time, EGFP expression co-localized with Tc-FoxQ2 
expression and showed almost completely overlap with it. It is worth mentioning that EGFP expression was completely overlapping with Tc-FoxQ2 expression in anterior neuroectoderm region where $\mathrm{Tc}^{-F o x Q 2}{ }^{+} \mathrm{NBs}$ had been identified, indicating EGFP also marked the Tc-FoxQ2 ${ }^{+} \mathrm{NBs}$. In addition, the expression of EGFP was also visualized in a small domain of the stomodeum where Tc-FoxQ2 expression was not detected (Figure 4.15B-B": dashed circles). This was suggested that EGFP marked more cells than Tc-FoxQ2 did.

At stage NS8, the expression pattern of Tc-FoxQ2 was not changed too much, with expression domains in the neuroectoderm and in the labral/stomodeal region (Figure 4.15C-C'"'). EGFP expression was consistent with Tc-FoxQ2 expression but showed very small domains without Tc-FoxQ2 expression. In the anterolateral region, 3 to 4 cells expressing EGFP were not Tc-FoxQ2 positive (Figure 4.15C-C': arrows), whereas the rest of EGFP positive cells were expressing Tc-FoxQ2.

From stage NS11 onwards, the expression of Tc-FoxQ2 in anterior neuroectoderm region successively approached the midline (Figure 4.15D-D"'), probably due to morphogenetic movements. EGFP expression was almost completely coincident with Tc-FoxQ2 expression. However, there was still a small group of EGFP positive cells in the anterolateral region not expressing Tc-FoxQ2 (Figure 4.15D-D": arrows). These cells were probably the same cells described at stage NS8 according to their location.

At stage NS13, Tc-FoxQ2 expression appeared in 4 cells in the ocular region while EGFP was not detected in these cells (Figure 4.15E-E": arrowheads). The expression of TC-FoxQ2 in anterior neuroectoderm region moved towards the midline, which was overlapping with EGFP (Figure 4.15E-E'"'). Additionally, a few cells located very close to the midline showed weak GFP signal but not Tc-FoxQ2 expression at that stage (Figure 4.15E-E": arrows).

At stage NS15, the latest embryonic stage, the initial brain lobes were formed. The protocerebrum was increased in size and the morphology of the protocerebrum section was visualized with DAPI staining. Tc-FoxQ2 showed a more complicated expression pattern in the protocerebrum. As shown in Figure 4.15F-F"', almost all the cells that expressed Tc-FoxQ2 were expressing EGFP as well. Two distinct cell groups were observed per hemisphere based on their distribution: the anterior median group and the lateral group (Figure 4.15F-F': dashed circles). The cells in the anterior median group stayed together to form a big column, which made the axonal projections to cross the midline and connected two brain hemispheres. I assumed that this group of cells would make contributions to the central brain formation. The other cells were distributed over larger areas and in spatially separate layers and their axonal projections were not visualized, which made it difficult to group these cells and determine the contributions of them. In addition, a complete Tc-FoxQ2 and EGFP co-expression was observed in the stomodeum, 
though Tc-FoxQ2 signal was not as strong as EGFP.

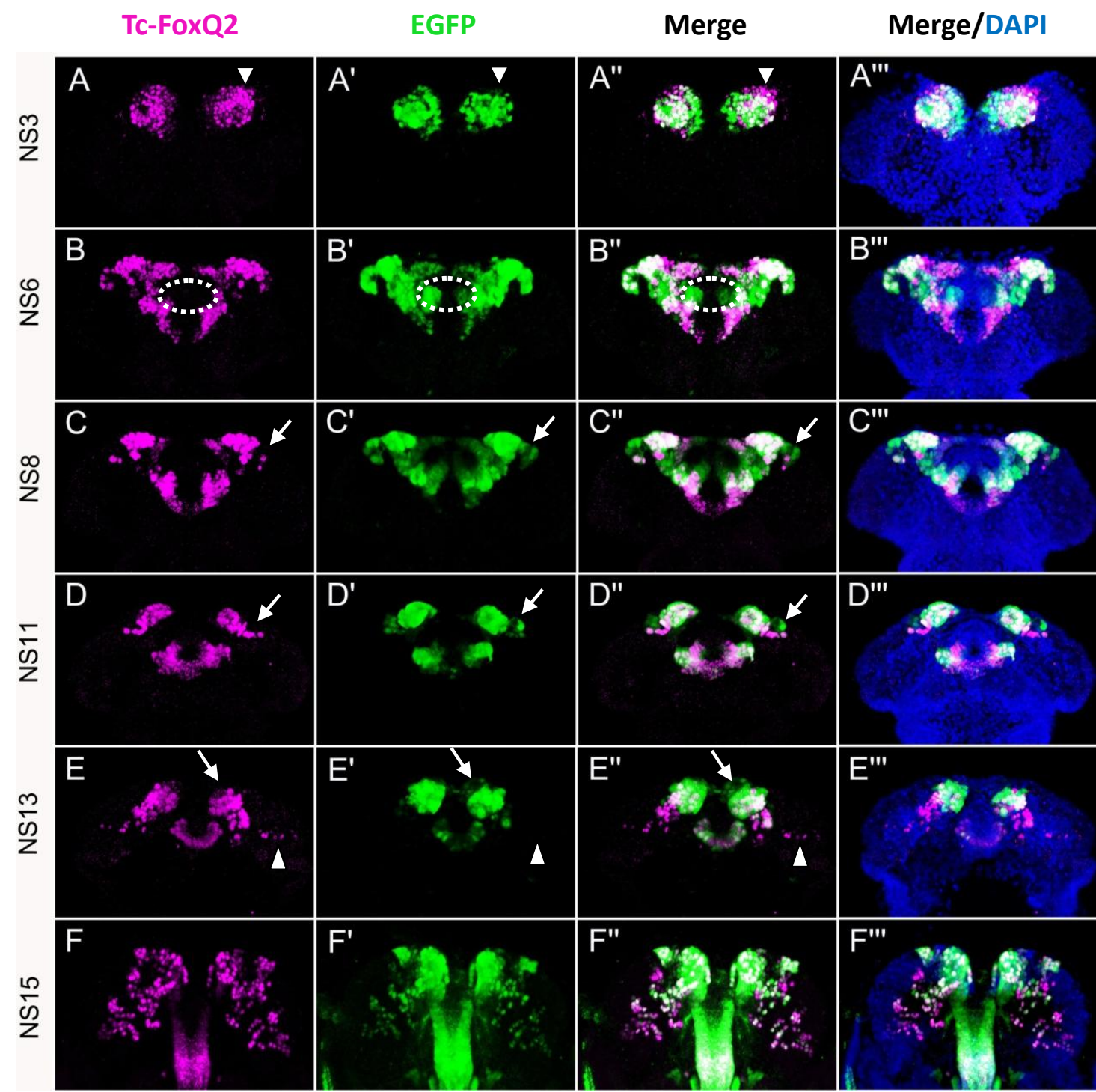

Figure 4.15 Overview of Tc-FoxQ2 and EGFP co-expression in the embryo. Almost all the cells that express Tc-FoxQ2 also express EGFP as well. All panels show maximum projection of all stacks. (A-A'"') At NS3, only few Tc-FoxQ2 positive cells in the anterior most outside are not expressing GFP (arrowheads). (B-B'") At NS6, co-expression is detected in the labrum, stomodeum as well as the anterior neuroectoderm region. Some cells in the stomodeum express EGFP but not Tc-FoxQ2 (dashed circles). (C-C'") At NS8, in the anterolateral region 3 to 4 cells expressing EGFP are not expressing Tc-FoxQ2 (arrows). (D-D'") From stage NS11 onwards, the expression of Tc-FoxQ2 in anterior neuroectoderm region successively approach midline. Several EGFP positive cells in the anterolateral region are not Tc-FoxQ2 positive (arrows). (E-E'") At NS13, a few cells located very close to the midline show weak EGFP signal but not Tc-FoxQ2 expression (arrows). Four cells in the ocular region only express Tc-FoxQ2 (arrowheads). (F-F"') At NS15, the assignment of the cells becomes more complicated. The dashed circles represent the anterior median group and the lateral group. 


\title{
4.4.2 Development and contribution of Tc-FoxQ2+ cells to the
}

\author{
central brain during embryogenesis
}

\subsubsection{Tc-FoxQ2 projects to the contralateral side through the initial axonal commissure in the embryo}

The brain commissures are tracts that connect the two brain hemispheres, where the cells extend their axons towards the midline. The axons from the same neural hemi-lineage often make their projections together. When these axons reach the midline, they fasciculate with their homologs and extend towards the opposite hemisphere. The primary axonal commissure in the brain is established by neurons that are located near the medial edge of each hemisphere and send their axons towards the midline (Therianos et al., 1995).

I used immunohistochemical markers and Tc-FoxQ2 enhancer trap line to track the temporal and spatial development of Tc-FoxQ2 positive cells during embryogenesis. Acetylated tubulin is known as a marker for axons of CNS (Black and Keyser, 1987; Bernhardt et al., 1990; Harzsch et al., 2002). By using anti-ac. tubulin staining, the axon tracts in the brain become visible during late embryonic development. I found that, at stage NS13 when the primary midline spanning axons appeared, a small cluster containing around 28 marked Tc-FoxQ2 cells was located at the medial edge of the hemisphere making contact with the axon tracts which crossed the midline ( $n=6$; Figure 4.16B: dashed lines, arrowheads). However, the axons that were extended by Tc-FoxQ2 cells and were supposed to be marked by EGFP were not detected (Figure 4.16A: arrowhead), which might be owing to the delayed EGFP expression in the enhancer trap line. This was suggested that these Tc-FoxQ2 cells projected through the primary commissure, which was probably built by other cells. Moreover, the cell cluster in question might be composed of one neural lineage (Figure 4.16A, B: dashed lines). This statement was based on the column like shape of the cells, which was typical for neural lineages such and the fact that I found one NB in that cluster recognized by its morphology and position that was distal to the axonal projection (Figure 4.16B: dashed circle).

Cells observed at the brain midline showing weak EGFP signal may be associated with the formation of the initial axonal commissure (Figure 4.16A, B: empty arrows). However, these cells were not expressing Tc-FoxQ2 protein (Figure 4.15E-E": arrows), indicating these cells shut off Tc-FoxQ2 but still had the stable EGFP, or the enhancer trap may drive non-Tc-FoxQ2 expression. Another group of cells located close to the posterior of the brain commissure moved towards superficial position in the mediodorsal brain during development (Figure 4.16A, B: 
stars). As they did not contribute to the central brain I excluded them from further characterization in this thesis.

Taken together, Tc-FoxQ2 cells projected to the contralateral side through the initial axonal commissure, but did not pioneer the primary brain commissure.
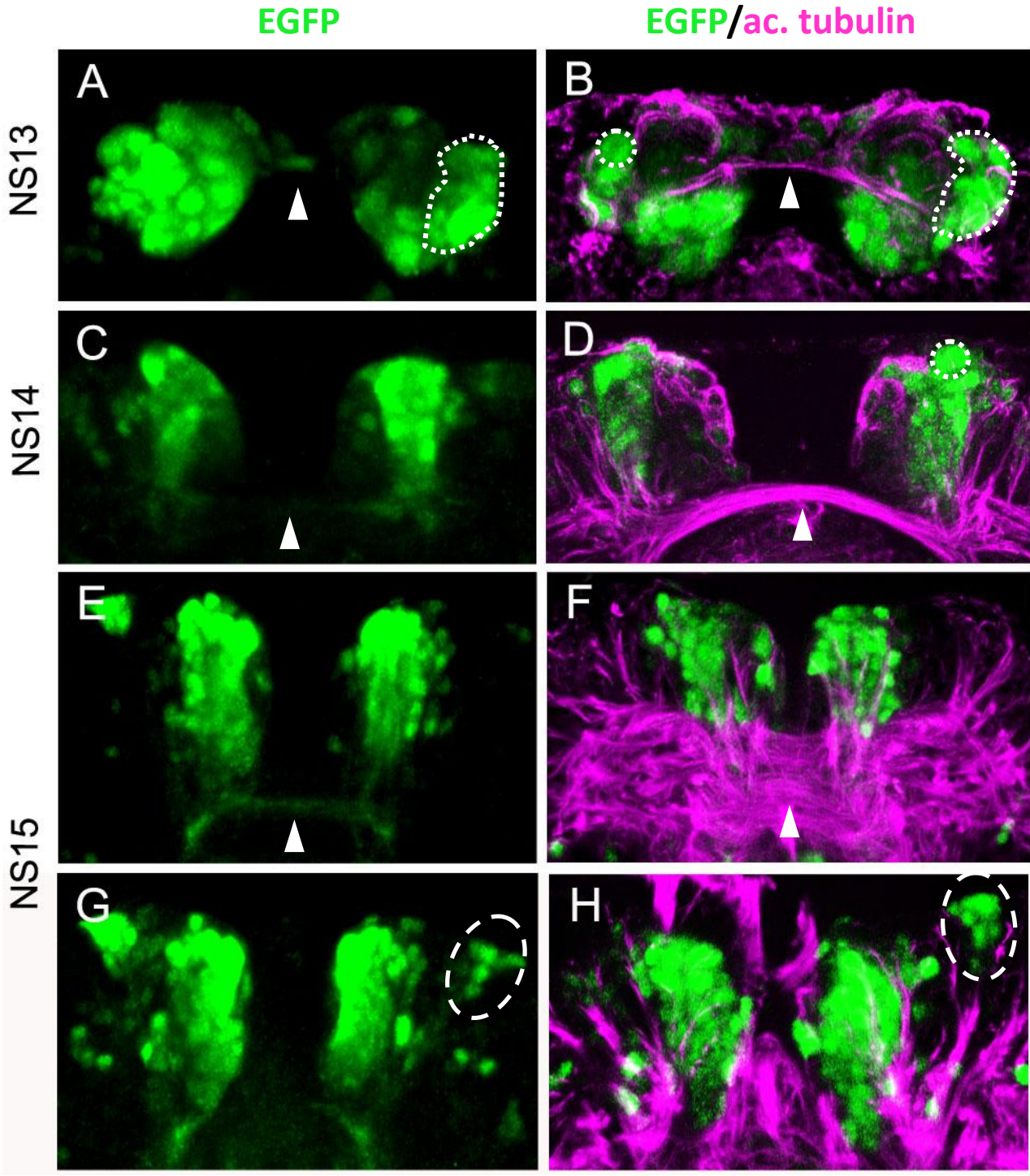

Figure 4.16 Development and contribution of Tc-FoxQ2 positive cells to the central brain during embryogenesis. (A, B) The initial axonal commissure forms at stage NS13 (B: arrowhead). The axons from Tc-FoxQ2 cells marked by EGFP are not detected (A: arrowhead). A small cluster of Tc-FoxQ2 cells might be composed of one neural lineage (white dashed lines). (C, D) At NS14, the median columnar lineage becomes larger with more cells. The axonal projections marked by EGFP from the lineage are detectable but not joined at the midline ( $C$ : arrowhead). More axon fascicles labeled by ac. tubulin generate and reach across the midline (D: arrowhead). (E, F) At NS15, the median columnar lineage produces more cells and moves closer to the midline. The axonal tracts marked by EGFP projected to the central brain primordium (E: arrowhead). The space in 
the brain midline was filled by numerous axons labeled by ac. tubulin (F: arrowhead). (G, H) The lateral lineage is detectable at NS15, showing a small cell cluster (orange dashed circle). Green: EGFP; magenta: ac. tubulin. (A) and (B), (C) and (D), (E) and (F), (G) and (H) are not the same embryo but from the same developmental stage.

\subsubsection{FAM2 neuroblast and A-PD neuroblast generate the}

\section{neural lineages contributing to the central brain primodium}

As the brain developed and underwent dynamic morphogenetic movements, the small lineage became a larger column-like lineage which progressively moved closer to the midline at stage NS14 (Figure 4.16C-F). At the same time, the cells belonging to this lineage remained together and differentiated into more cells throughout development, forming a large and compact cluster. The NB which generated the specific lineage was recognized easily (Figure 4.16D: white dashed circle) and the number of the progeny increased, revealing 37 to 43 cells in each hemisphere $(n=5$; Figure $4.16 C, D)$. Axonal projections marked by EGFP from these cells were observed but at that stage did not yet join at the midline (Figure 4.16C: arrowhead). Immunohistochemical analysis using ac. tubulin antibody revealed that, more axon fascicles were generated and crossed the midline, forming tight axon bundles (Figure 4.16D: arrowhead). Several fibers that derived from the neural lineage projected into two bundles (Figure 4.16D: arrow), reaching the midline by following the axonal pathways.

At stage NS15, the median lineage contained approximately 65 cells in each hemisphere that was small and densely packed ( $n=4$; Figure 4.16E, F). However, I was unable to trace and identify the NB at this stage due to massive number of cells and lack of specific NB markers. The axons formed one fascicle and projected into a coherently-labeled midline structure which possessed the morphological features of the central brain primordium (Figure 4.16E: arrowhead). The lateral lineage showed a small cluster containing around 15 cells ( $n=4$; Figure 4.16G, H: orange dashed circles). The axonal tracts generated by the lateral lineage projected into the midline. Anti-ac. tubulin staining revealed that the commissural axons differentiated further to become complicated and massive. The space in the brain midline was filled by numerous axons (Figure 4.16F: arrowhead). Four axon fascicles in each hemisphere (Figure 4.16F: arrow) originated from the median columnar lineage and projected into the central brain.

As Tc-FoxQ2 ${ }^{+}$NBs had been identified during embryogenesis (see section 4.3.2), I wondered, which of the identified NBs generated the median columnar lineage and the lateral lineage. Based on its position, I hypothesized that the FAM2 NB 
which was previously identified at stage NS14 could generate the median columnar lineage based on the following criteria: Both FAM1 and FAM2 were NBs located close to the midline and they were located closely to each other. The FAM1 NB produced few offspring that showed weak EGFP signal and the axon fascicles were barely detected (Figure 4.17A-A": dashed lines). By contrast, the FAM2 NB (Figure 4.17A-A": dashed circles) generated more progeny and gave rise to recognizable lineage. The A-PD NB located laterally to the FAM2 NB could be the one producing the lateral lineage (Figure 4.17B-B": dashed circles). At stage NS14, A-PD NB lineage was small with only few cells.

Taken together, by using Tc-foxQ2 enhancer trap line and ac. tubulin antibody, I found that Tc-FoxQ2 cells projected through the initial axonal commissure in the embryonic brain. I also found that the FAM2 NB generated the median columnar lineage and A-PD NB generated the lateral lineage, which projected axon fascicles into the central brain primordium. Further, this Tc-FoxQ2 positive projection prefigures the $C X$ as will be shown in subsequent section.
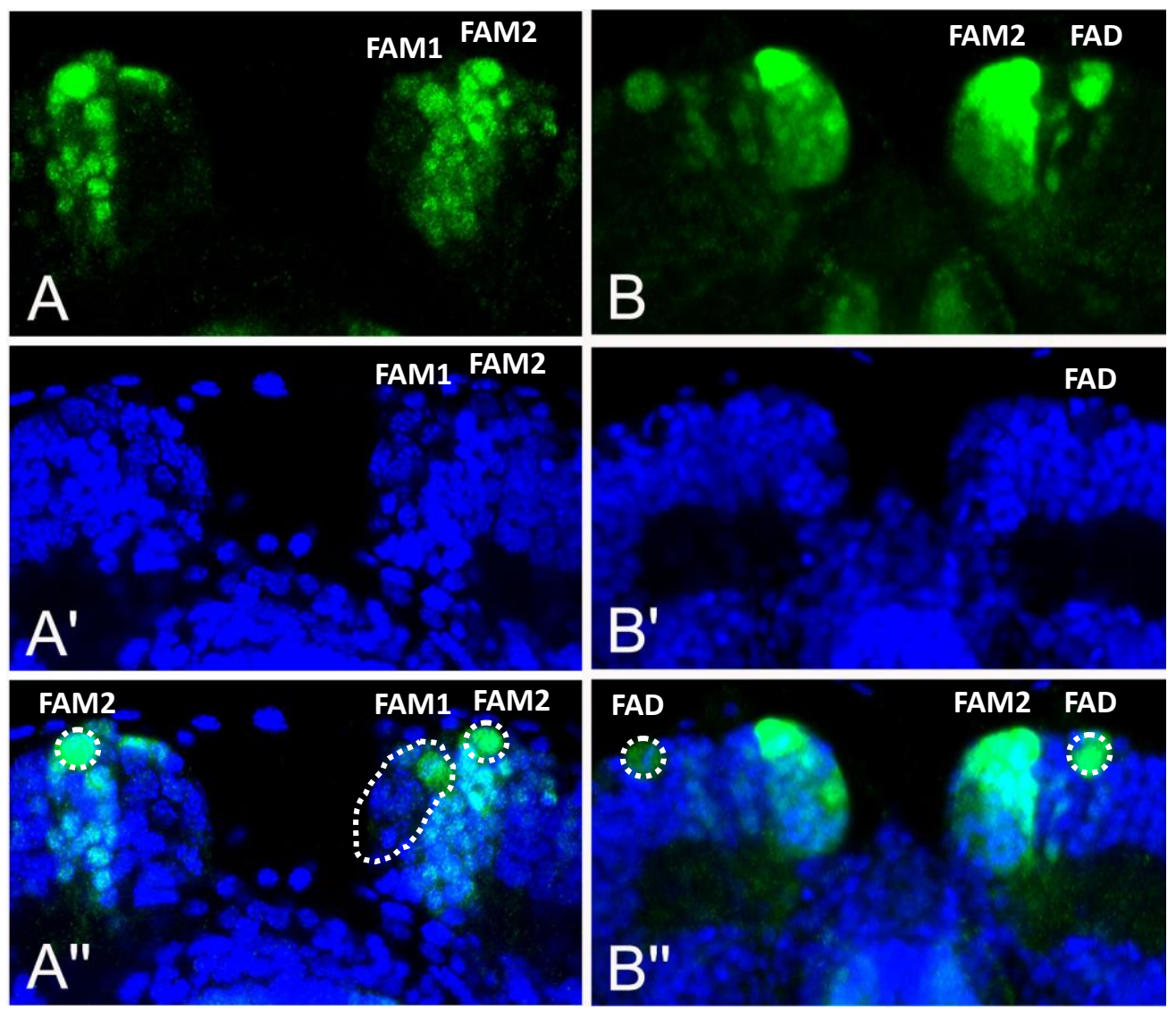

Figure 4.17 The FAM1 NB, FAM2 NB and A-PD NB lineages at stage NS14. (A-A') FAM1 NB generates a small amount of offspring that show weak EGFP signal (white dashed line). FAM2 NB generates the columnar lineage. (B-B") A-PD NB lineage is small with only few cells. DAPI staining is used to visualize the cell nuclei. The white dashed circles represent the NBs. Green: EGFP; blue: DAPI. 


\subsubsection{Tc-FoxQ2+ cells are not glial cells}

It is known that the NBs generate both neurons and glia. I wondered if Tc-FoxQ2 positive cells included also glial cells. Immunohistochemical staining was performed with an antibody against the glial marker reversed polarity (anti-Repo; Campbell et al., 1994; Lee and Jones 2005). However, I observed that not a single Tc-FoxQ2 cell from NS13 to NS15 overlapped with Repo staining, indicating that Tc-FoxQ2 cells are indeed neurons and not glial cells (data not shown).

\subsubsection{Development and contribution of Tc-FoxQ2+ cells to the central brain postembryonically}

\subsubsection{Development and contribution of Tc-FoxQ2 ${ }^{+}$cells in the \\ larval brain}

In the larval brain, three cell clusters were observed in each hemisphere: the median cluster, the lateral cluster, and the posterior cluster (Figure 4.18A-C: white circle, white dashed circle and blue circle). In the median cluster, not all Tc-FoxQ2 cells expressed EGFP, but almost all EGFP cells were Tc-FoxQ2 positive (Figure 4.18A-C: white dashed circle). The cells co-expressed Tc-FoxQ2 and EGFP were dispersed spatially in different layers. The complete co-localization of Tc-FoxQ2 and EGFP were found in the cells of lateral cluster which was the most distal to the midline (Figure 4.18A-C: white circle). Some of the cells in the lateral cluster sent the axon tract to the most posterior commissure (Figure 4.18B: empty arrows). The cells in the posterior cluster (Figure 4.18A-C: blue circle) generated bundles of fibers and contributed to the other less posterior commissure (Figure 4.18B: empty arrows). I assumed that these cells corresponded to the posterior group of the embryo based on their position (Figure 4.15F-F"). Owing to their location, I could eliminate the possibility that the two posterior brain commissures belong to the central complex (CX).

Two anterior brain commissures which might be related to the central brain according to their position were observed (Figure 4.18D: arrow, arrowhead). The lateral cluster contained 17 to 21 cells and generated a bundle of fibers projecting into the outside of the central body (CB) anterior rim ( $n=3$; Figure 4.18D, F: arrows), presumably the protocerebral bridge $(\mathrm{PB})$ based on its position. The $\mathrm{CB}$ coated by glial cells was marked with DAPI (Figure 4.18E: yellow dashed circle; Koniszewski et 
al., 2016). According to the position and projection of the lateral cluster, I assumed that it corresponded to the lateral lineage of the embryo (Figure 4.16G, H: orange dashed circles). In addition, the other commissure was observed at the posterior rim of the CB (Figure 4.18D, F: arrowheads). However, I was unable to trace this commissure back to the cells the projections came from. Moreover, the cells in the median region were arranged not compactly (Figure 4.18B: white dashed circle). Based on their position, they might be related to the most anterior commissure (Figure 4.18D: arrow). However, their projections were not detectable during the larval stage. These cells might correspond to the median columnar lineage of the embryo based on their location (Figure 4.16E). It was shown that the median columnar lineage of the embryo containing approximately 65 cells at NS15. However, the median region of the larva showed reduced cell number, which might undergo the apoptosis and reorganization during the transition.

Taken together, in the larval brain all EGFP positive cells were Tc-FoxQ2 positive as well, but not all cells expressing Tc-FoxQ2 were EGFP positive. The cells co-expressed Tc-FoxQ2 and EGFP in the lateral cluster were shown to contribute to the central brain, presumably the PB based on its position.
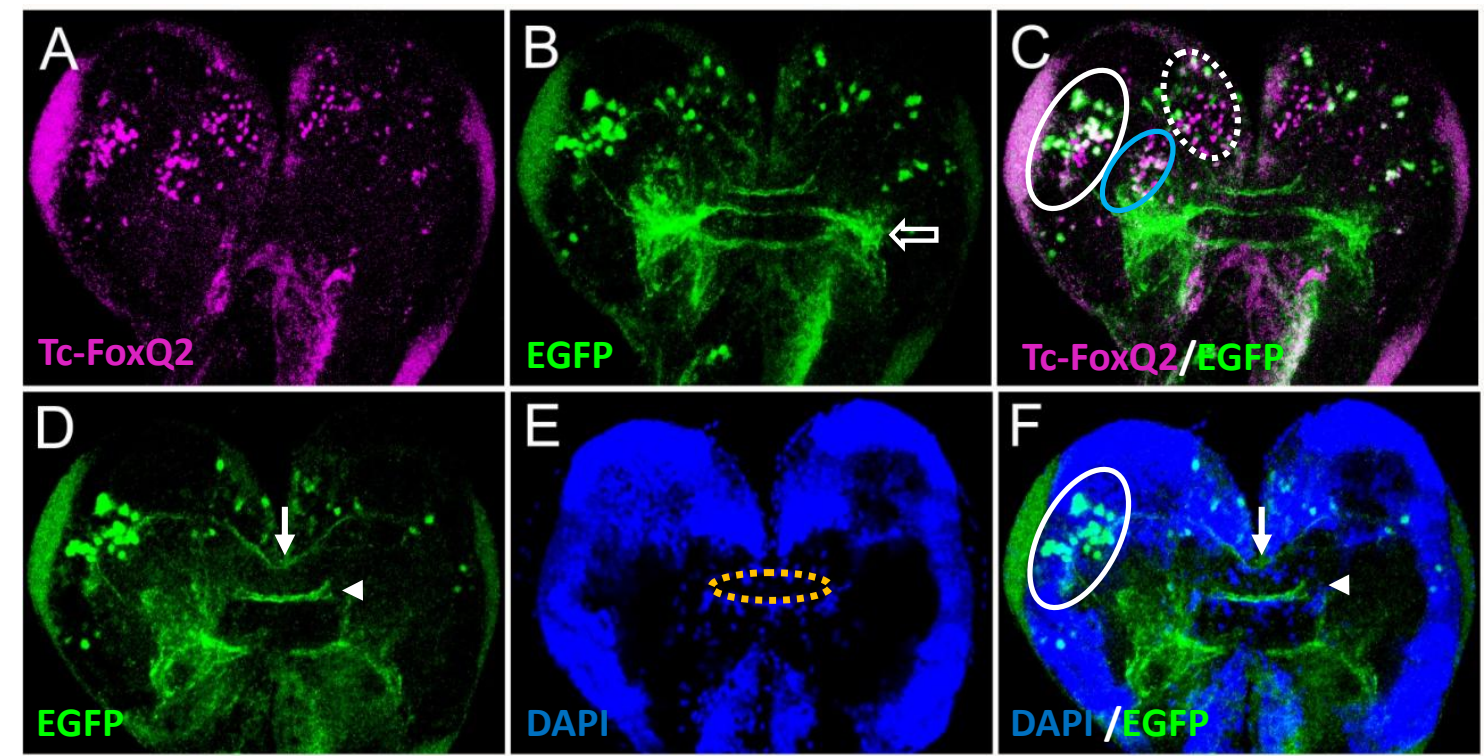

Figure 4.18 Contribution of Tc-FoxQ2 cells to the central brain in the larval brain. (A-C) Co-expression of TC-FoxQ2 and EGFP in the larval brain. (C) is the merge of (A) and (B). All panels show the maximum projection of all stacks. Three clusters of cells are in each hemisphere: the lateral cluster (white circles), the median cluster (white dashed circles) and the cluster located between the midline cluster (blue circles). The lateral cluster shows the complete co-localization of Tc-FoxQ2 and EGFP. The two posterior commissures (empty arrows) originate from the lateral cluster and the cluster between the median cluster and lateral cluster. (D-F) The lateral cluster projects into the central brain. (F) is the merge of (D) and $(E)$. The panels show the maximum projection of selected stacks. The CB coated by glial cells is marked with DAPI (orange dashed circle). Green: GFP; magenta: Tc-FoxQ2; blue: DAPI. Anterior is up in all panels. 


\subsubsection{Development and contribution of Tc-FoxQ2+ cells in the}

\section{adult brain}

In adult brain, the expression pattern and projection pattern were similar to the larval brain. Three clusters of cells were observed on either side of the midline and the vast majority of the cells showed the co-expression of Tc-FoxQ2 and EGFP (Figure 4.19A-A": white circle, white dashed circle and blue circles). The cells in the anterior cortex only expressed Tc-FoxQ2, no EGFP signal were detected in these cells (Figure 4.19A-A": arrowheads). The lateral cluster that contained 20 to 25 cells co-expressing Tc-FoxQ2 and EGFP ( $n=3$; Figure 4.19A-A": white circle) made more bundles of fibers project across the midline (Figure 4.19A': arrow), which were located anterodorsal to the $\mathrm{CB}$ and seemed to innervate into the PB (Figure 4.19B-B": white dashed outline and arrows). The cells in the median cluster sent three to four long tracts through the PB and straight towards the posterior (Figure 4.19C- $\left.C^{\prime \prime}\right)$. The posterior cluster contained fewer cells co-expressing Tc-FoxQ2 and EGFP, only two to three of which were EGFP positive (Figure 4.19A-A": blue circles). These cells made their projections to the unknown axons across the midline in the posterior (Figure 4.19A': empty arrow), which probably corresponded to one of the posterior commissures in the larval brain (Figure 4.18B: empty arrow). Additionally, the anterior part of the CB was marked with EGFP (Figure 4.19D-D"). However, it was impossible to track back to the respective cell bodies. Interestingly, an unknown U-shape structure which made central brain commissures was detected (Figure 4.19E-E": arrowheads). This structure was located ventrally to the CB and therefore not relevant to the $\mathrm{CB}$, but again, it was impossible to track back to the respective cell bodies. I assumed that this structure probably developed from the commissures at the posterior rim of the $C B$ in the larval brain (Figure 4.18D, F: arrowheads). DAPI staining was used to visualize the borders of the $\mathrm{PB}$ and the $C B$ (Figure 4.19 B', D': white dashed outlines). Moreover, compared to the larval brain, the cell number as well as their spatial distribution did not change too much in the adult brain, indicating that the larval brain neurogenesis generated the vast majority of the adult brain.

Taken together, EGFP and Tc-FoxQ2 were highly overlapping and EGFP marked the subset of Tc-FoxQ2 cells in the adult brain. The cells co-expressed EGFP and Tc-FoxQ2 in the median cluster and lateral cluster generated interesting axon bundles which projected into the PB. Tc-FoxQ2 cells and axonal projections directly innervating to the $\mathrm{CB}$ were not observed. 

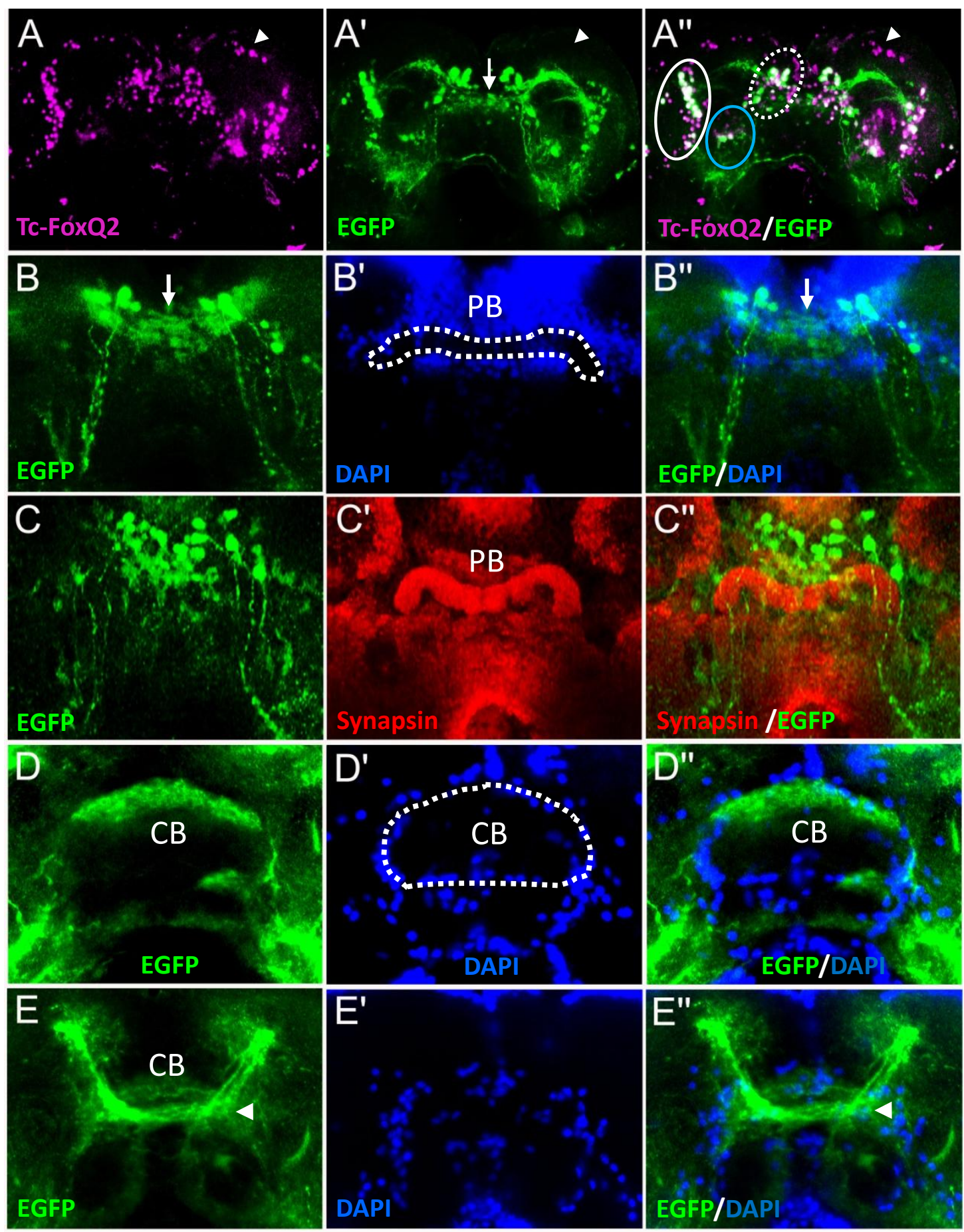

Figure 4.19 Contribution of Tc-FoxQ2 cells to the central brain in the adult brain. (A-A") Co-expression of Tc-FoxQ2 and EGFP in the adult brain. Three clusters of cells are observed in each brain hemisphere, showing the almost completely overlap of Tc-FoxQ2 and EGFP. (B-B") The fiber bundles from the lateral cluster project together crossing the midline, which appears to innervate the PB (arrows). (C- $C^{\prime \prime}$ ) The cells in the median cluster sent three long tracts initially to the $\mathrm{PB}$, and then almost vertically towards the posterior. (D-D") TC-FoxQ2 marks the upper unit of the CB. (E-E') An unknown U-shape structure projects across the central brain (arrowheads). Green: EGFP; magenta: Tc-FoxQ2; red: Synapsin; 
blue: DAPI. DAPI staining enables the visualization of borders of the PB and CB (white dashed outlines). Anterior is up in all panels.

\subsection{Tc-foxQ2 Knock-down causes central brain phenotypes}

\section{in the embryo}

It has been shown that $T c$-six 3 is required for the $C X$ formation. Tc-six3 knock-down led to defects in the CB in L1 larvae (Posnien et al., 2011b). Further, Tc-foxQ2 and $T c$-six3 are largely overlapping in the anterior neuroectodem and Tc-foxQ2 also has a function in neural development, resulting in the size reduction of the $C X$ in Tc-foxQ2 RNAi animals (Kitzmann et al., 2017). Tc-FoxQ2 and Tc-six3 were co-expressed in the neuroectoderm during embryogenesis and Tc-FoxQ2 positive NBs were shown to be $T c$-six3 positive (see section 4.3). In order to investigate the neural function of Tc-foxQ2 in more detail, I performed Tc-foxQ2 pRNAi in the SB strain and different imaging lines which mark the central brain (raw counts are displayed in Table 7.5-7.14).

\subsubsection{Tc-foxQ2 RNAi in the $T c$-foxQ2 enhancer trap line}

The 86up line is an enhancer trap line which was generated in this work (see section 4.2) and marked Tc-foxQ2 expressing cells with EGFP. Tc-foxQ2 RNAi in this line showed that the signal intensity of neuroectodermal EGFP expression was strongly reduced compared to the stomodeal expression (Figure 4.20). At stage NS13, the pattern of the EGFP expression domain appeared not to be reduced but the signal intensity and the number of marked cells was strongly reduced $(n=5$; Figure 4.20A, B: arrows; Figure 4.20E). Interestingly, I found that one neural lineage in each brain hemisphere was reduced (Figure 4.21A, B: circles) and the NB was still there in RNAi animals (Figure 4.21 $\mathrm{A}^{\prime \prime}$, $\mathrm{B}^{\prime \prime}$ : dashed circles). According to its location, it could be the FAM2 lineage (see section 4.4.2.2). At stage NS15, the number of the marked cells in the median brain was obviously reduced $(n=4$; Figure 4.20D: arrow; Figure 4.20E) compared to the wt ( $n=4$; Figure 4.20C: arrow; Figure 4.20E). The cells that were still marked belonged mainly to the median columnar lineage that was described in section 4.4.2.2. The axonal projection from these cells was strongly reduced, showing only one tract crossing the brain midline (Figure 4.20D: dashed box; Figure 4.20D": arrowhead). In wt, the axonal projections from the cell group formed a bundle of tracts across the midline (Figure 4.20C: dashed box; Figure 4.20C": arrowhead). In addition, the EGFP expression in the stomodeum was not altered - it showed a strong signal and its regular shape (Figure 4.20C, D: empty arrows). The difference in Figure $4.20 \mathrm{C}$ versus Figure $4.20 \mathrm{D}$ was due to 
different orientation of the embryo. Taken together, Knock-down of Tc-foxQ2 in the Tc-foxQ2 enhancer trap line resulted in the reduction of Tc-FoxQ2 expression indicating a self-regulatory function of this transcription factor.
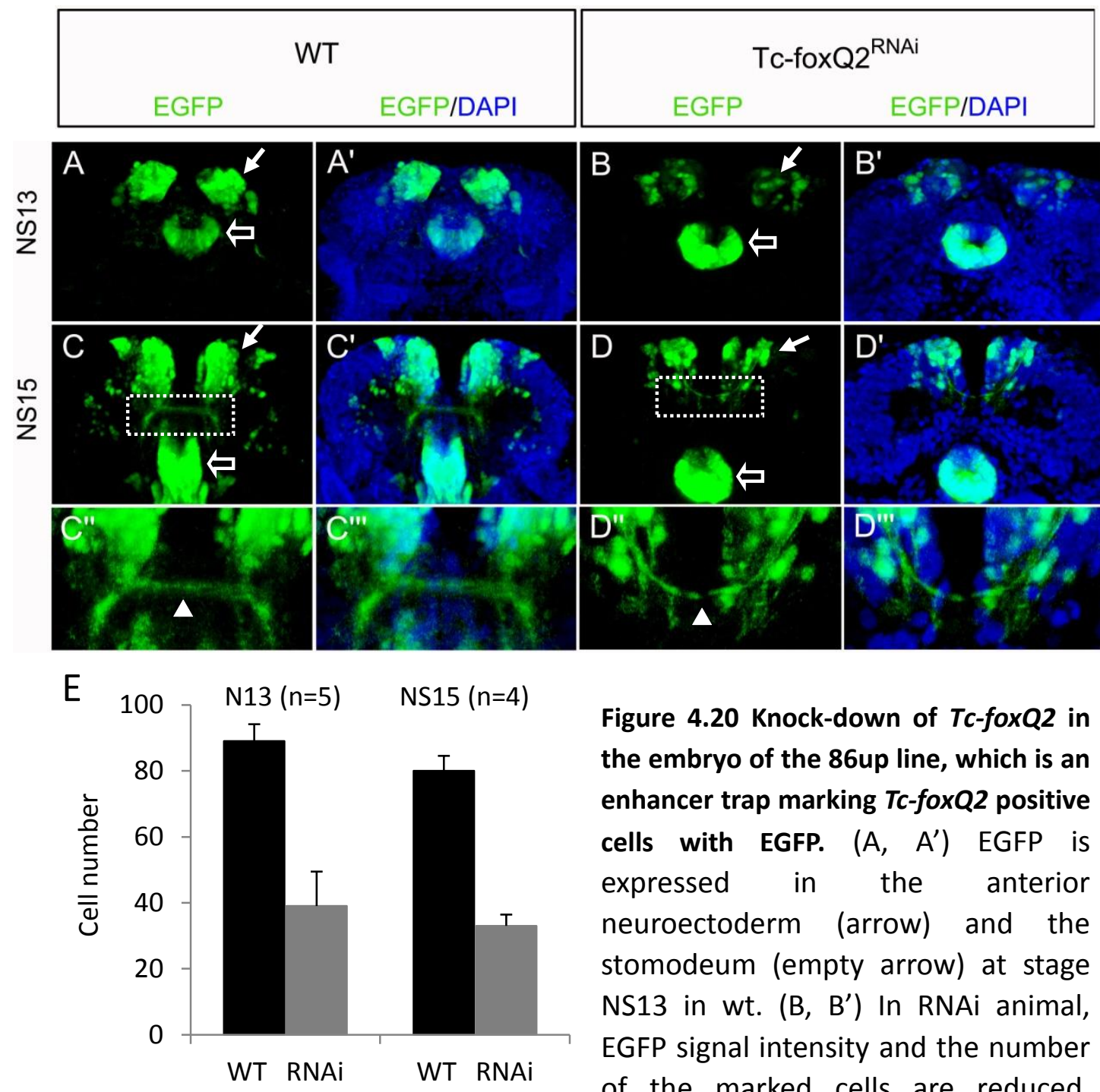

Figure 4.20 Knock-down of Tc-foxQ2 in the embryo of the 86up line, which is an enhancer trap marking $T c$-foxQ2 positive cells with EGFP. (A, $\left.A^{\prime}\right)$ EGFP is expressed in the anterior neuroectoderm (arrow) and the stomodeum (empty arrow) at stage NS13 in wt. (B, $\left.B^{\prime}\right)$ In RNAi animal, EGFP signal intensity and the number of the marked cells are reduced.

(C- $C^{\prime}$ ) shows the marked cells in the anterior brain (arrow) and the stomodeum (empty arrow) at stage NS15 in wt. The axonal tracts from these cells in the anterior brain are crossing the midline (dashed box). (D-D') In RNAi animal, the marked cells in the anterior brain (arrow) and the axonal tracts across the midline (dashed box) are significantly reduced. $\left(C^{\prime \prime}-C^{\prime \prime \prime}\right)$ and $\left(D^{\prime \prime}-D^{\prime \prime \prime}\right)$ show the close-ups of the brain commissures (arrowheads) in ( $\left.C-C^{\prime},\right)$ and (D-D') (dashed boxes), respectively. (E) The total cell number in the neuroectoderm is reduced at NS13 and NS15 in RNAi. Green: EGFP; blue: DAPI. 

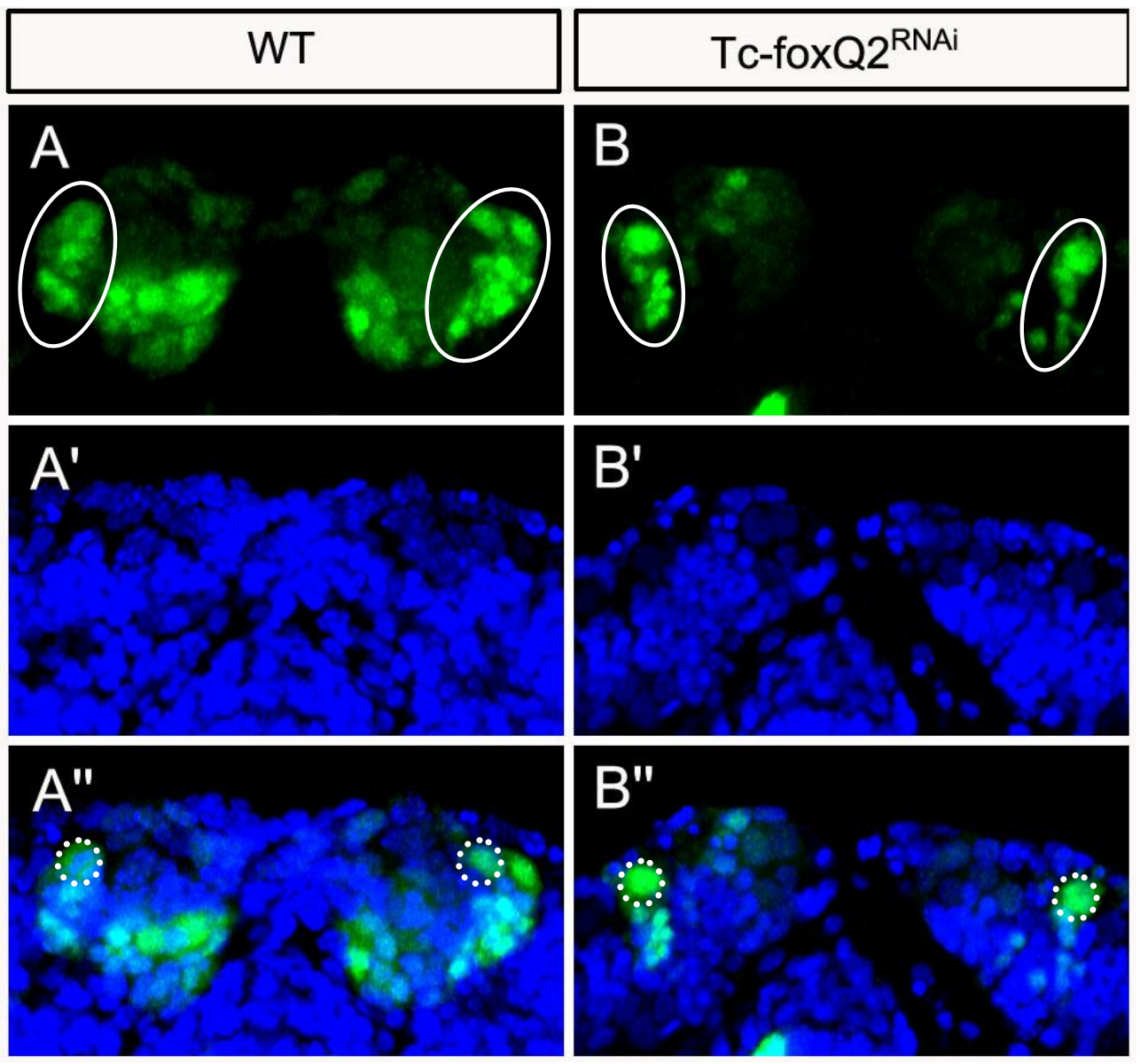

Figure 4.21 Knock-down of $T c$-foxQ2 leads to a reduction of the neural lineage at stage NS13 in the 86up line. (A- $\left.A^{\prime \prime}\right)$ In wt, one NB lineage is shown in the anterior neuroectodermal region in each hemisphere (circle). The dashed circle represents the NB. (B-B") In RNAi animal, the lineage is reduced (circle) and the NB remains there (dashed circle). Green: EGFP; blue: DAPI.

\subsubsection{Tc-foxQ2 RNAi in the $S B$ strain}

To investigate the role of $T c-f o x Q 2$ in the development of axonal projections in the brain, ac. tubulin staining was performed in the $S B$ embryos. By using DAPI, the morphology of the brain sections was visualized. At stage NS13, the initial midline spanning axons appeared in wt (Figure 4.22A, $A^{\prime \prime}$ : arrowheads; section 4.4.2.1). In RNAi, the initial brain commissures were still detectable and even not reduced (Figure 4.22B, B": arrowheads). Further, it showed a groove in the anterior median region of the brain (Figure 4.22B- $\mathrm{B}^{\prime \prime}$ : arrows) compared to the wt (Figure 4.22A-A": arrows). This defect might be the reduction or loss of the labrum since previous study has showed that Tc-foxQ2 RNAi could lead to the severe defect in the labrum (Kitzmann et al., 2017). This was suggested that Tc-foxQ2 was not required for the formation of the initial brain commissures. In stage NS15 of wt, at least seven 
axonal fascicles originated from the anterolateral brain and projected through the brain midline (Figure 4.23A). When these axons reached the midline, some of them apparently connected with their homologs from the other hemisphere. It appeared that some of them extended towards the opposite hemisphere forming a cross at the midline (Figure 4.23A: arrowhead). The Knock-down of Tc-foxQ2 resulted in central brain defects. The axonal projections were disordered and did not follow the stereotypic pathways to cross the midline (Figure 4.23B: arrow), which led to shorter and asymmetric brain commissures (Figure 4.23B: arrowhead). The brain hemispheres appeared to be more close to each other, showing a shorter space in the central brain (Figure 4.23B': arrowhead) in line with previous findings (Kitzmann et al., 2017). This phenotype was observed most frequently. Another phenotype showed disordered axonal projections in the brain (Figure 4.23C: arrow) and only one bundle of the commissures in the central brain (Figure 4.23C: arrowhead). Further, the brain hemispheres seemed to be heavily reduced along the anteroposterior axis in this phenotype. Taken together, these data in $S B$ showed an important and widespread role of Tc-foxQ2 in the development of projections of the brain. Tc-foxQ2 is not required for the formation of the initial brain commissures, only for the later brain commissures.

ac. tubulin
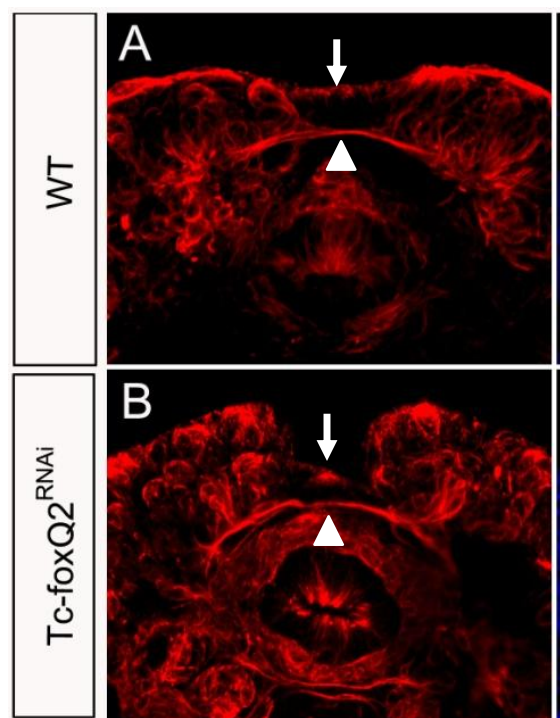

DAPI
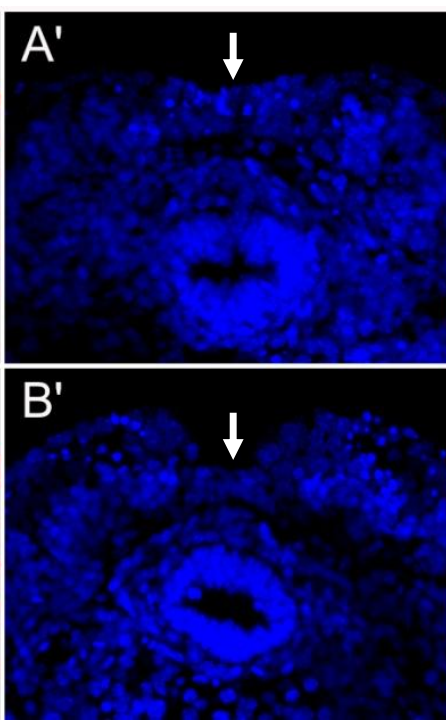

ac. tubulin/DAPI

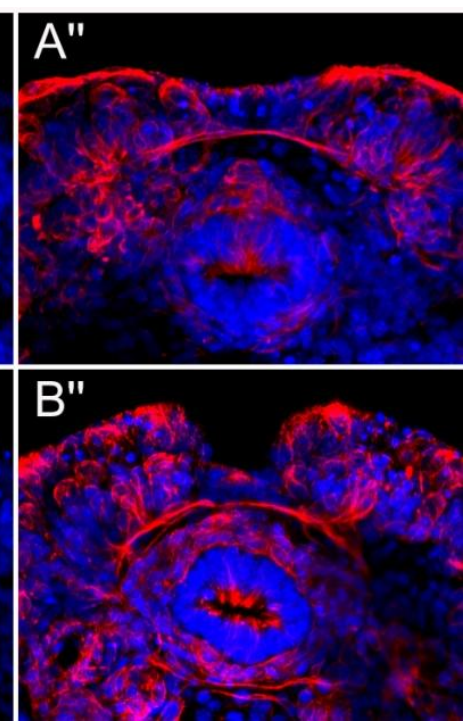

Figure 4.22 Knock-down of Tc-foxQ2 in the embryo of $S B$ at stage NS13. (A-A') In wt, the initial brain commissures form at stage NS13 (arrowheads). (B-B") Tc-foxQ2 RNAi shows no aberrations in the initial brain commissures (arrowheads) but a groove in the anterior median domain of the brain (arrows). Red: ac. tubulin; blue: DAPI. 
ac. tubulin
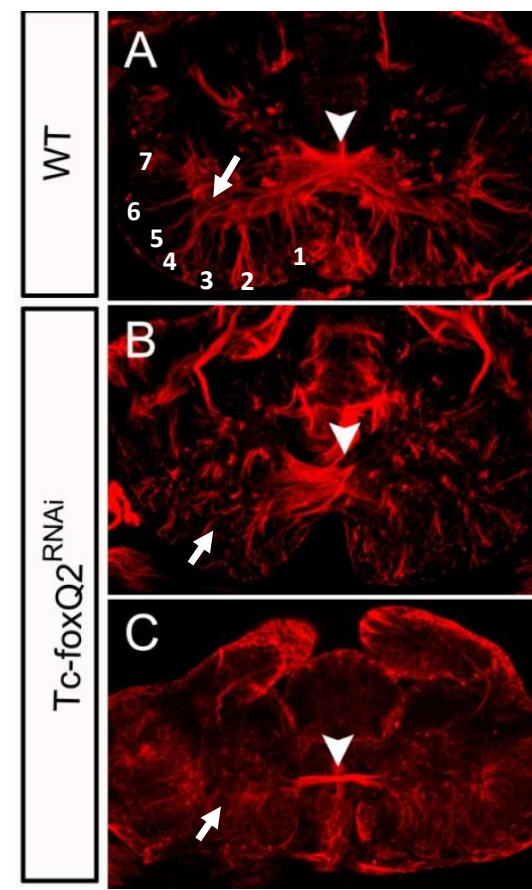

DAPI
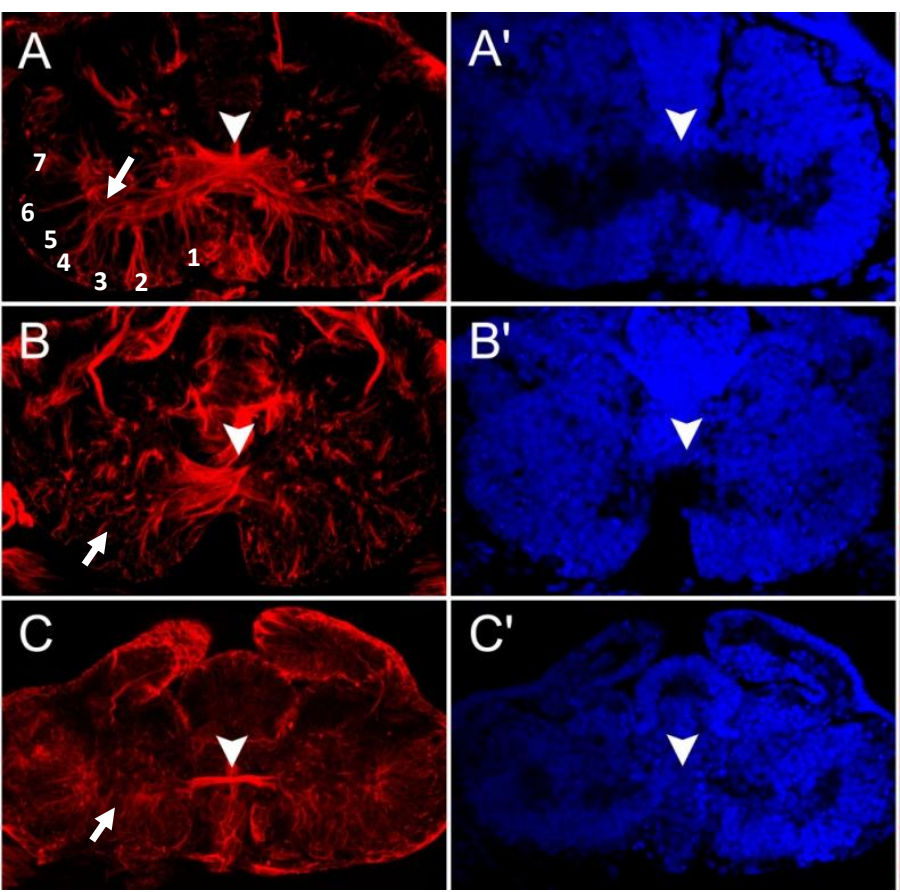

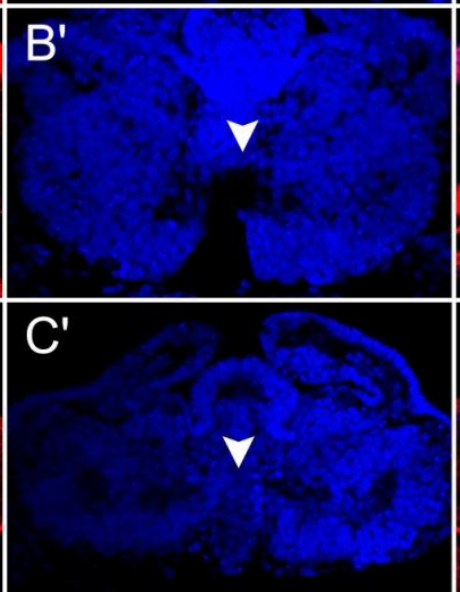

ac. tubulin/DAPI

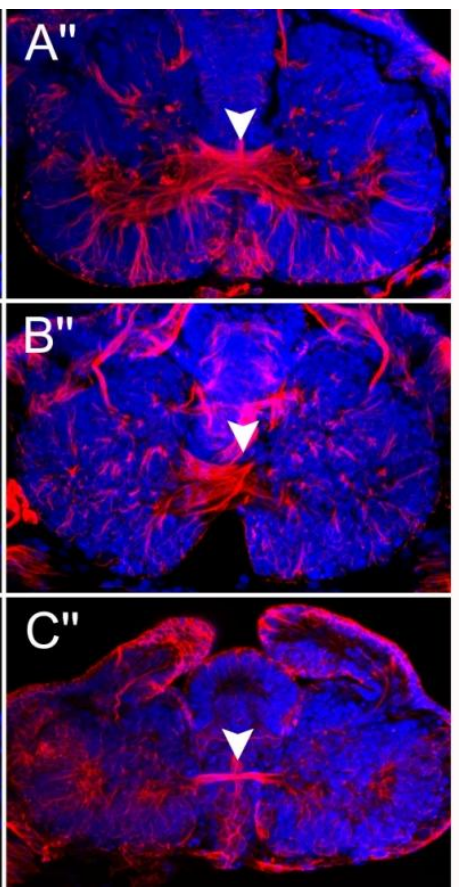

Figure 4.23 Knock-down of $T c$-foxQ2 in the embryo of SB at stage NS15. (A-A") In wt, at least seven axonal bundles in the anterolateral brain (arrow) project through the brain midline (arrowhead). (B- $\mathrm{B}^{\prime \prime}$ ) and (C- $\mathrm{C}^{\prime \prime}$ ) shows the phenotypes in the central brain in RNAi animals. The brain commissures are reduced ( $\mathrm{C}$ : arrowheads) and the axonal projections show the disarrangement ( $\mathrm{B}, \mathrm{C}$ : arrows; $\mathrm{B}$ : arrowhead). The brain hemispheres are more close to each other $\left(B^{\prime}\right)$ or are reduced along the anteroposterior axis $\left(C^{\prime}\right)$. Red: ac. tubulin; blue: DAPI.

\subsubsection{Tc-foxQ2 RNAi in the imaging lines marking the central}

\section{brain}

The line E035004 (lab internal number: 120) is an enhancer trap line derived from the GEKU screen, in which a construct containing EGFP under the control of a 3xP3 promoter is inserted (Berghammer et al., 1999; Trauner et al., 2009). This line has shown to be in the Tc-ten- $a$ locus and the signal is overlapping with anti-Ten-a staining (Marita Buescher's work). In Drosophila, Ten-a is a dimeric receptor which marks the axons of the embryonic CNS (Fascetti and Baumgartner, 2002). In this line, three groups of cells marked with EGFP were observed at stage NS15. The median group contained $\sim 27$ cells ( $n=4$; Figure 4.24A: dashed circle) while the lateral group contained approximately 32 cells ( $n=4$; Figure 4.24A: empty arrow). 
However, the projections of these two groups were too fused to follow. The posterior group possessing $\sim 39$ cells projected through the midline $(n=4$; Figure 4.24A: circle, arrowhead), which was shown to be a subset of the brain commissures marked with ac. tubulin (Figure 4.24A': arrowhead). Knock-down of Tc-foxQ2 most frequently led to the disarrangement of cells in the central brain and the loss of the marked part of the brain commissures (Figure 4.24B: arrowhead). The number of the cells in the posterior group was reduced and many of these cells showed weakened EGFP signal (Figure 4.24B: circle). The axonal projections from this posterior group into the midline were missing. The cells in the median group seemed to be misarranged in the central brain (Figure 4.24B: dashed circle). The lateral group remained invariant but showed weaker EGFP signal (Figure 4.24B: empty arrow). Note, that the brain commissures marked with ac. tubulin were not completely gone (Figure 4.24B": arrowhead) and the brain hemispheres appeared to be closer similar to the findings shown above (Figure 4.24B": arrows), which was in consistent with the observation of RNAi in SB (Figure $\left.4.23 \mathrm{~B}-\mathrm{B}^{\prime \prime}\right)$. In the strongest phenotype, the brain commissures were completely absent, and the brain hemispheres were reduced in size and were fused at the midline (Figure 4.24C-C"': arrowheads, arrows) such that the assignment of cells to the respective groups becomes difficult. The putative cells in the posterior group appeared to be separated into two groups (Figure 4.24C: circles) due to the heavily changed brain hemisphere and their projections were not detectable. Moreover, the cells of the putative median and the lateral groups became closer and the number of the cells in these two groups was decreased (Figure 4.24C: dashed circle, empty arrow). 


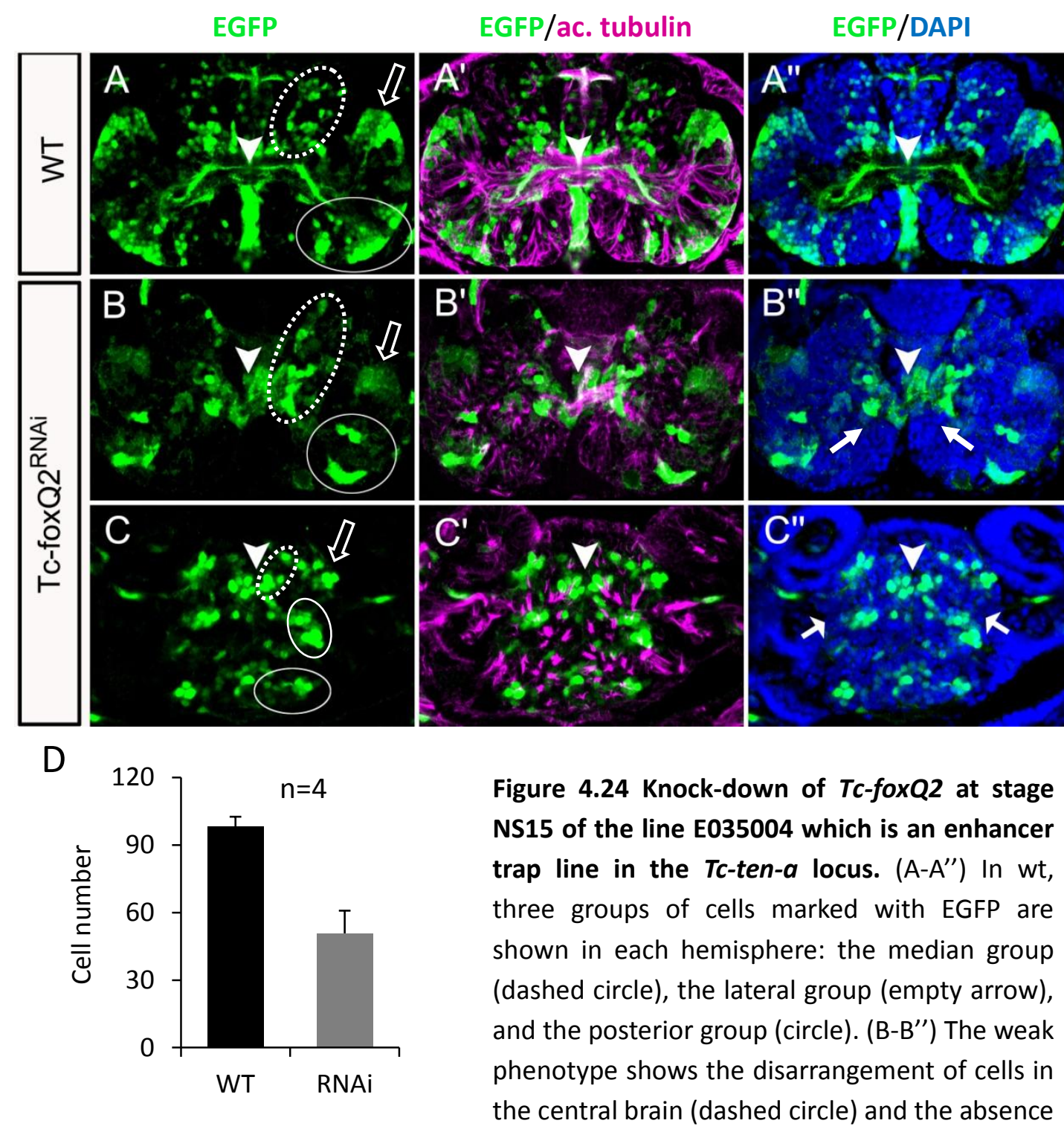

of the marked part of the brain commissures (arrowhead). The cells in the posterior group are reduced (circle) and their axonal projections towards the midline are missing. (C-C") The strongest phenotype shows the fused brain hemispheres and the strongly reduced brain size (arrows). The brain commissures are completely absent (arrowheads). (D) The total cell number of the three groups is reduced in RNAi. Magenta: ac. tubulin; green: EGFP; blue: DAPI.

The line E011A-01 (lab internal number: 50) is a Tc-rx enhancer trap line from the GEKU screen. The insertion is known to be in the $5^{\prime}$ upstream region of Tc-rx. In this line, two groups of cells marked with EGFP were observed to contribute to the central brain. The median group contained $\sim 43$ cells which made three to four axonal projections across the midline ( $n=6$; Figure 4.25A: circle), while the lateral group contained $\sim 34$ cells which sent two tracts across the midline ( $n=6$; Figure 4.25A: dashed circle). Tc-foxQ2 Knock-down in this line showed that the brain 
commissures were reduced (Figure 4.25B: arrowheads) and the cell number of the median group and the lateral group was significantly decreased as well (Figure $4.25 \mathrm{C}$ ). In RNAi animals, the median group projected only one tract through the midline (Figure 4.25B: arrow). The axonal tracts from the lateral group were barely seen. The brain hemispheres were shown to be closer to each other and the space in the central brain where the brain commissures located was reduced (Figure 4.25B': arrowhead).

EGFP
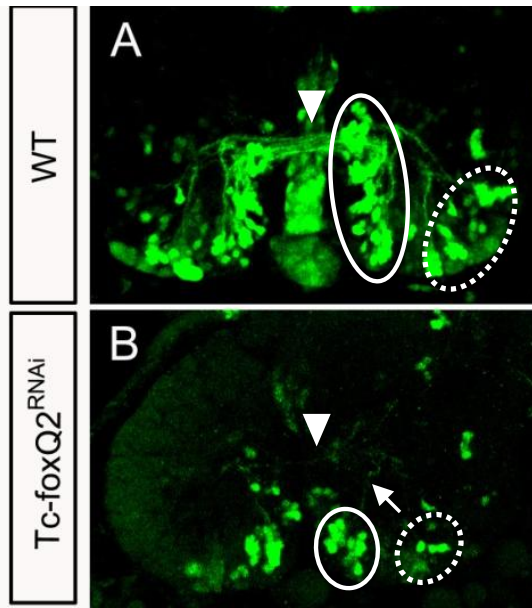

C

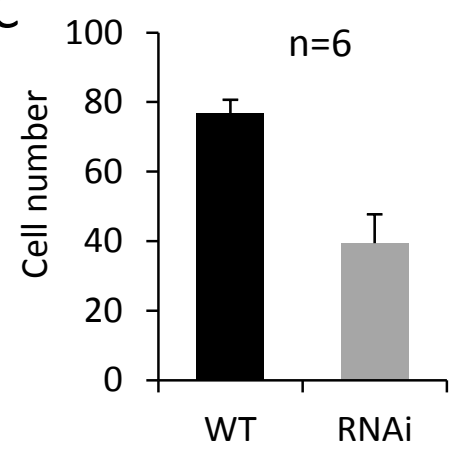

DAPI

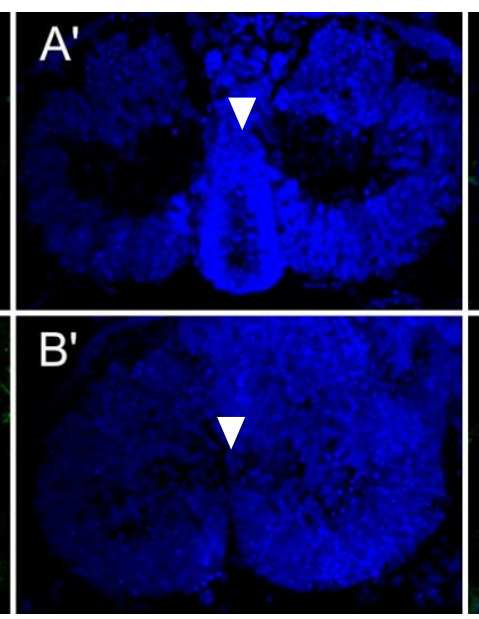

Figure 4.25 Knock-down of Tc-foxQ2 at stage NS15 in a Tc-rx enhancer trap line. (A-A') In wt, two groups of the cells marked with EGFP (circle, dashed circle) make the projections to the central brain (arrowhead). (B-B") The phenotype shows a strongly reduced brain commissures (arrowhead) and reduced cells contributing to the central brain (circle, dashed circle). (C) The total cell number of the two groups is reduced in RNAi. Green: EGFP; blue: DAPI.

Tc-rx reporter line (lab internal number: 178) contains parts of the upstream genomic region of $T c-r x$ fused with basal promoter elements driving DsRedExpress (DsRedEx) (Koniszewski, 2011). This line marked one median group of cells contributing to the central brain (Figure 4.26A: circle, arrowhead). A lateral group of cells did not contribute to the central brain (Figure 4.26A: arrow) and the axons from these cells were not detectable. Knock-down of Tc-foxQ2 most frequently led to an obviously reduced cell numbers and the complete loss of the brain commissures (Figure 4.26B: circle, arrowhead). Interestingly, the cells located in the lateral rim of the brain hemispheres were almost absent although they did not project to the central brain (Figure 4.26A, B: arrows). The brain hemispheres appeared to be more closely (Figure $4.26 A^{\prime}, B^{\prime}$ ). 

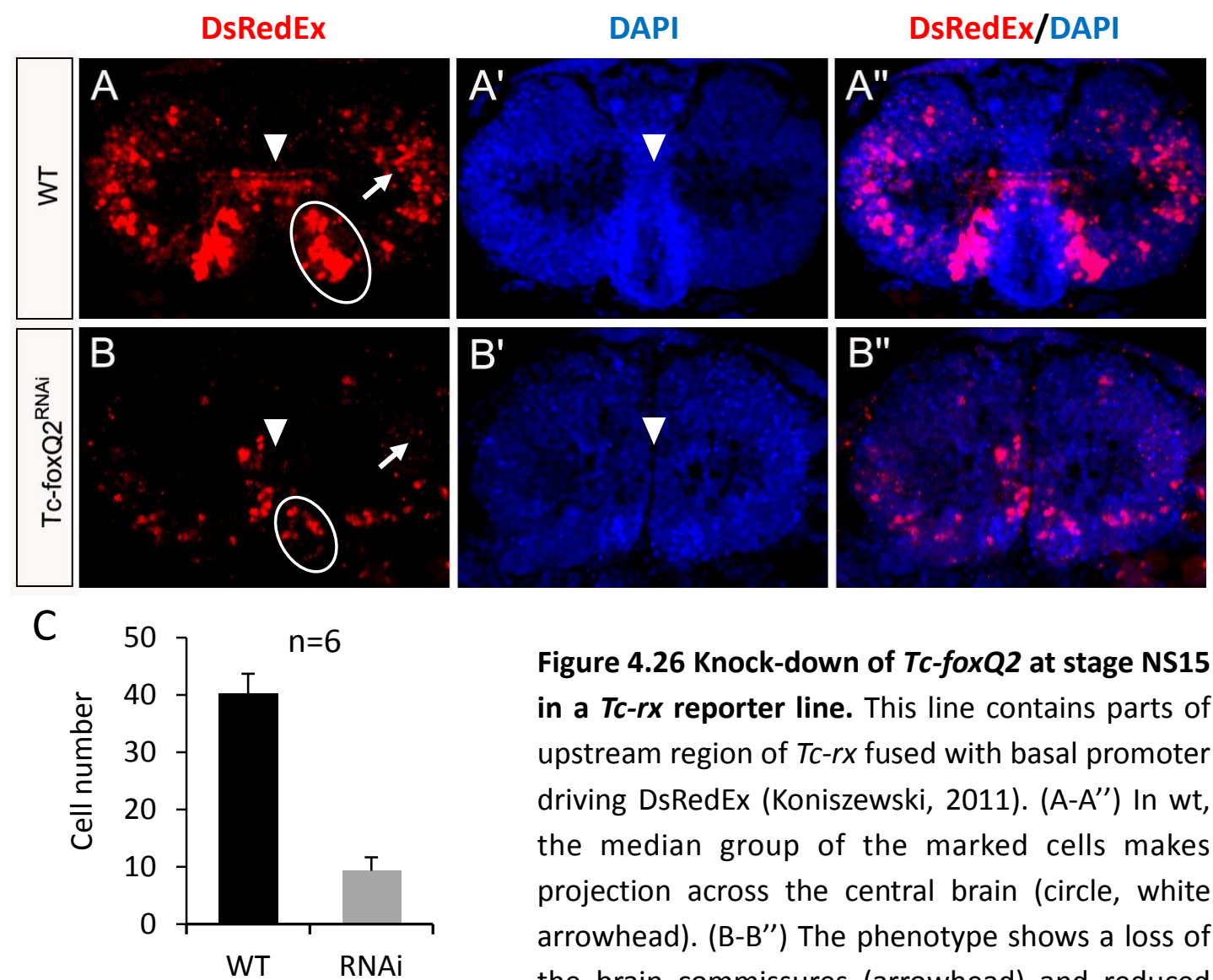

Figure 4.26 Knock-down of Tc-foxQ2 at stage NS15 in a Tc-rx reporter line. This line contains parts of upstream region of $T c-r x$ fused with basal promoter driving DsRedEx (Koniszewski, 2011). (A-A") In wt, the median group of the marked cells makes projection across the central brain (circle, white arrowhead). (B-B") The phenotype shows a loss of the brain commissures (arrowhead) and reduced

cells contributing to the central brain (circle). The number of the cells located in the lateral rim is also reduced (arrow). (C) The cell number of the median group is significantly reduced in RNAi. Red: DsRedEx; blue: DAPI.

Taken together, the experiments using the brain imaging lines demonstrate that Tc-foxQ2 is required for the formation of the central brain. Knock-down of Tc-foxQ2 leads to the aberrant formation of the brain commissures at later stage (NS15), but does not affect the formation of the initial brain commissures at earlier stage (NS13). Tc-foxQ2 is absolutely required for the formation of midline crossing projections of a number of cell groups (e.g. in line E035004, E011A-01, Tc-rx 178) but it does not appear to act on the top level, because some projections are still visible in RNAi embryos based on ac. tubulin staining (Figure 4.23B, C: arrowheads). Further, I find a medial fusion of the brain hemispheres as described before (Koniszewski, 2017) and the reduction of a number of cells which contribute to the central brain (e.g. in line line E035004, E011A-01, Tc-rx 178). Further, the reduction of EGFP signal and number of marked cells in the Tc-foxQ2 enhancer trap line indicates a self-regulatory function of this gene. 


\section{Discussion}

Drosophila is the most studied model insect for brain development. However, due to the absence of the $C X$ in the embryonic stage, the early determinants and signals required for $C X$ development remain unknown. The red flour beetle Tribolium has become the second most studied model insect in developmental biology after Drosophila. It exhibits a more typical developmental process of the insect, like the short germ mode of segmentation, development of larval legs and the formation of a non-involuted head. Importantly, the CB partially forms during the embryogenesis in Tribolium but respective neuropils are developing only at late larval and pupal stages in the fly. Hence, the embryonic development of the central brain is quite diverged in Drosophila such that Tribolium is a good model for studying this process. Here, I used Tribolium to study the early brain development, focusing on the $\mathrm{CX}$ and central brain development. I generated a Tc-FoxQ2 antibody and an enhancer trap line to mark Tc-foxQ2. Further, I identified Tc-FoxQ2 ${ }^{+} \mathrm{NBs}$ and their lineages which contributed to the $\mathrm{CX}$ development. Moreover, I found that Tc-FoxQ2 ${ }^{+}$NBs were marked by different combinations of transcription factors. Finally, Tc-foxQ2 RNAi showed that Tc-foxQ2 played an important role in the axon scaffold formation and was required for the central brain probably including $\mathrm{CX}$ development.

\subsection{CRISPR/Cas9}

\subsubsection{CRISPR/Cas9 mediated knock-in via non-homologous end joining (NHEJ)}

In order to study the contribution of Tc-foxQ2 positive cells to the brain I needed to specifically mark those cells. The strategy that I used was to generate a Tc-foxQ2 enhancer trap line via CRISPR/Cas9. Compared to the conventional approaches which rely on the piggyBac transposon mediated integration of the construct randomly into the genome (Trauner et al., 2009; Koniszewski, 2011), CRISPR/Cas9 technology allows for inserting the construct at a specific location (Garneau et al., 2010; Jinek et al., 2012; Gilles et al., 2015), which makes the process easier and more efficient. Homology directed repair (HDR) and non-homologous end joining (NHEJ) are two main approaches to integrate the marker construct into the target locus. The reasons why I used NHEJ are on the one side, the same construct can be 
re-used for any gene of interest. On the other side, it has been proven that NHEJ is the predominant DNA repair mechanism and occurs more frequently than HDR (Gilles and Averof, 2014). In the zebrafish, the CRISPR/Cas9 mediated knock-in via NHEJ showed a very high efficiency of germ line transmission, which could reach 34.2\% (Auer et al., 2014). Further, even if NHEJ is less precise compared to HDR, the enhancer trapping occurs in the non-coding DNA (upstream region or in the first intron) but not in the exon, which means the accuracy is actually not required. Thus, NHEJ as a feasible approach for knock-in was applied in this study.

\subsubsection{The survival rate of injected embryos and the}

\section{concentrations of injection components}

In the CRISPR/Cas9 experiments, in order to increase the possibility of double stranded break (DSB), I designed multiple gRNAs, which I mixed for targeting the same region (the upstream region or the first intron). In one treatment, the plasmid was just linearized at one position. Here, the survival rate of the injected animals of the upstream treatment (15.64\%) which had two gRNAs targeting sites was higher than the first intron treatment (14.41\%) which had three gRNAs targeting sites (Table 4.2). In the other treatment, the plasmid was cut twice in order to remove the backbone from the construct. In this treatment, the survival rate of the injected animals of the upstream treatment $(21.88 \%)$ was also higher than the first intron treatment (19.26\%). This could be owing to the amount of injected plasmids. Generally, more injected components leads to higher lethality. Alternatively, it could be that the increased likelihood of inducing DSBs with three gRNAs might lead to more damage and consequently death of the animal. Moreover, with more gRNAs the likelihood of off target effects might increase.

For the same targeting region and using the same gRNAs, however, the plasmid cut twice which had one more gRNA showed the higher survival rate than that of the plasmid cut once. In the first intron, the survival rate of the injected animals of the plasmid cut twice is $19.26 \%$, while the survival rate of the injected animals of the plasmid cut once is $14.14 \%$. In upstream treatment, the plasmid cut twice also showed higher survival rate of the injected animals $(21.88 \%)$ than the plasmid cut once (15.64\%). Again, the high concentration of injected components could have affected the survival rate. However, it is also possible that technical issues affected injection quality.

\subsubsection{The efficiency of CRISPR/Cas9 in Tribolium}

I finally got nine positive lines, which were all from the linearized repair plasmid 
without removing the backbone. Six lines came from the treatment targeting the upstream region while three came from the treatment targeting the first intron. The efficiency of germ line transmission was 2.6\% (G0 with positive offspring compared to all surviving G0) for the upstream and $1.3 \%$ for the first intron, respectively (Table 4.2). This was generally consistent with the results of Schwertner's work, which showed the efficiency between $1.7 \%$ to $3.9 \%$ (Schwertner 2016, unpublished). In the zebrafish, the efficiency of germ line transmission of the knock-in via NHEJ ranged from 1.2\% to 34.2\% (Auer et al., 2014).

Of these nine transgenic lines, only one (No.86) from the upstream region showed the Tc-FoxQ2 expression pattern in its offspring (section 4.4.1). The three transgenic lines in the first intron showed a similar EGFP expression pattern in the anterior median region (Table 4.3). This indicates that probably the insertions of these three lines are in the same locus and might have the same orientation. Although the EGFP expression was in the anterior median region, it did not show a pattern identical to the Tc-FoxQ2 expression pattern. Thus, I did not analyze it further. It would be interesting to analyze the expression between Tc-FoxQ2 and EGFP by antibody staining with this line, e.g. how far the expression overlaps or if the EGFP expression is a subset of Tc-FoxQ2 expressing cells. In the upstream region, except for the line 86up which showed a pattern very similar to the endogenous Tc-FoxQ2 expression pattern, other lines showed an unexpected EGFP expression or no EGFP signal (Table 4.3). One reason for the different patterns could be the different orientation of the insertion at the same location, which could possibly change the influences of the surrounding enhancers and could lead to the different regulatory activity. Due to the fact that the backbone is still present, the promoter of the construct is either quite close to the endogenous promoter (i.e. 700 bp upstream of the transcription start site) while it is $\sim 7.5 \mathrm{~kb}$ apart in the other orientation. Indeed, the line 86up is oriented such that the promoter is close to the endogenous one.

Further, the different insertion location, i.e. gRNA1 or gRNA2 within the upstream region, could result in different expression patterns because of the different location with respect to the surrounding enhancers. If both gRNA1 and gRNA2 worked at the same time, the entire part between them ( $620 \mathrm{bp})$ would be lost. Moreover, the insertion could also have occurred at an off-target site and produce a different pattern for that reason. Although the evaluation of the gRNAs has been done at the beginning with a bioinformatics tool, this could only minimize the potential off-target effects and not completely eliminate them. Since the line 86up with expected Tc-FoxQ2 expression was available and the integration was shown to be as expected, the molecular nature of the other lines was not analyzed further.

I did not get any positive transgenic individuals from the treatment in which the 
plasmid was cut twice to remove the backbone. The reason could be as follows: after linearizing two linear DNA fragments were generated: the backbone and the construct. The backbone would compete with the pure construct for knock-in, which would lead to the reduction of the chances for the expected pure construct. It would be possible that the backbone was inserted but was not identified due to the lack of the marker.

In summary, by using CRISPR/Cas9 mediated knock-in via NHEJ, I successfully generated a $T c$-foxQ2 enhancer trap line. In these experiments, the germ line transmission was in the range of other reports but not specifically high (1.3\%, 2.6\%). In the zebrafish, the efficiency of germ line transmission of knock-in via NHEJ could reach $34.2 \%$ (Auer et al., 2014). The reasons for the lower germ line transmission rate might be the time delay of the Cas9 and gRNA transcription from the plasmid. It is unknown when and how efficiently the gRNA is transcribed after injection, even if an endogenous promoter is used. The same is true for Cas9. For increasing the germ line transmission efficiency, it would be possible to inject gRNA and Cas9 as mRNA or inject the linearized repair plasmid. It would be also possible to generate a transgenic line expressing Cas9 under the germ line specific driver, similar to nanos or vasa in Drosophila (Ren et al., 2013; Sebo et al., 2014) . The intention of injecting multiple gRNAs together is to increase the possibilities of the DSB, because not all gRNAs work efficiently. However, when combined with NHEJ to integrate a repair plasmid, complicated insertion can happen, e.g. the orientation of the repair plasmid, the multiple copies of the repair plasmids, which make it difficult to identify and understand the insertion. Moreover, the chance of off-target may increase with more gRNAs and the intervening fragment could insert in the other orientation, which could lead to changes in regulation. Therefore, injecting only one efficient gRNA at a time might be a good alternative. The T7 Endonuclease assay can be used to test the efficiency of gRNAs but it does not exactly reflect the in vivo situation. Regarding the quite good reflection of Tc-FoxQ2 expression by the enhancer trap generated in this work, this approach might be useful to generate reporters for other genes in the future.

\subsection{The development of Tc-FoxQ2+ cells to the central}

\section{brain}

\subsubsection{The enhancer trap line is a good tool to study the}

\section{development of Tc-FoxQ2+ cells}

In order to study the brain development, neural cells building the brain need to be 
visualized. Only this way, the question of how NBs generate the corresponding lineages and develop into the neuropils at a cellular level in the brain can be answered. To this end, transgenic lines marking a specific genes involved in the brain development can be generated (Koniszewski et al., 2016). In this study, I generated an enhancer trap line ( $T c$-foxQ2up) which marked the Tc-FoxQ2 positive cells by EGFP. The investigation for the overlap of EGFP and Tc-FoxQ2 in Tc-foxQ2up line showed an almost complete consistence in the embryo, larva and adult (section 4.4). However, still few cells showed divergent expression. Some cells only expressed EGFP but not Tc-FoxQ2, indicating these cells shut off Tc-FoxQ2 but still had the stable EGFP, or the enhancer trap may drive expression in non-Tc-FoxQ2 cells. Other cells were only Tc-FoxQ2 positive but did not show EGFP expression. Either EGFP expression in Tc-foxQ2up line was delayed or the enhancer trap did not mark the expression in these cells. Taken together, despite a low degree of unexpected expression, the Tc-foxQ2up line is a good tool to study the development of Tc-FoxQ2 positive cells. In a parallel project by Max Farnworth in the lab, a bicistronic line was generated in Drosophila where the marker is fused to and expressed from the endogenous transcript. Here, a close to $100 \%$ overlap was observed. Hence, this approach is more exact but also much more labor intensive.

\subsubsection{Tc-FoxQ2 lineages contribute to the central complex}

\section{development}

In this work, I identified Tc-FoxQ2 ${ }^{+} \mathrm{NBs}$ in the embryonic stage and successfully traced two of them and their lineages into the adult stage. The two Tc-FoxQ2 ${ }^{+} \mathrm{NBs}$, FAM2 and A-PD, were found to contribute to the CX development. They gave rise to a median lineage and a lateral lineage in the brain, respectively (section 4.4.2; 4.4.3). It is known that the vast majority of cell bodies in the larval brain remain during metamorphosis to build the adult brain (Ito and Hotta, 1992; Maurange et al., 2008). Hence, it is expected that the larval brain and adult brain basically have similar structural characteristics. Indeed, in Tc-foxQ2up line, the larval brain and the adult brain showed similar EGFP expression patterns and could be linked intuitively based on the position and projection of the cells. This means the corresponding cell clusters in the adult brain can be also found in the larval brain. Three clusters of cells were observed in each hemisphere in both larval and adult brain: a median cluster, a lateral cluster and a posterior cluster (Figure 4.18C; Figure $4.19 \mathrm{C})$. In the adult brain, the cells in the median cluster projected their axonal tracts through the $\mathrm{PB}$ and then extended towards the posterior (Figure 4.19C-C'). The upper unit of the CB was marked by EGFP, however, no cell bodies and projections could be unequivocally assigned to this signal (Figure 4.19D-D'). A comparison with other species might be helpful in this respect: in the grasshopper, median cell lineages project their axonal bundles initially into the PB and then 
reach the $C B$, connecting the $P B$ and $C B$ by $W, X, Y, Z$ tracts (Boyan and Williams, 1997; Williams et al., 2005; Williams and Boyan, 2008; Boyan et al., 2008; Boyan and Reichert, 2011). Therefore, I assume that the contribution of the marked upper unit of the CB possibly originated from the median cluster. However, due to the low number of tracts and technical limitations, e.g. the quality of the immunostaining or the resolution of the imaging, it was impossible to see and trace back these projections. In the larval brain, the cells in the median cluster were separately arranged and the axonal projections were not detected (Figure 4.18B, C: white dashed circles). Interestingly, in the last embryonic stage, the cells in the median cluster formed a big stereotypic column and projected across the brain midline (Figure 4.16E: arrowhead). Based on these observations, I hypothesize that some cells might undergo apoptosis and the remaining ones might rearrange during the transition from the embryo to the larva. To test this speculation, caspase antibody staining could be performed to check for apoptosis (Florentin and Arama, 2012).

It is known in Drosophila that the NBs generated in the embryonic stage are responsible for the larval neurogenesis after undergoing a period of quiescence during transition (Truman and Bate, 1988; Prokop and Technau, 1991; Ito and Hotta, 1992; Maurange et al., 2008). However, in this work, due to the lack of the molecular marker for NBs and the technical limitations, NBs in the larval brain, even in the last embryonic brain, could not able to be identified. To get a better understanding of the correlation of NBs and their lineages in the embryo and larva, it would be interesting to perform immunostainings in L1 larval brains with NB markers, like anti-Ase and anti-Dpn protein. However, several attempts by others in the lab of generating an anti-Ase antibody were not successful.

The lateral cluster tangentially sent the axonal bundles across the midline and appeared to innervate the PB in the adult brain (Figure 4.19B-B": arrows). It is known that the tangential neurons typically arborize within a single subunit of the CX (Homberg 1985; Hanesch et al., 1989; Loesel et al., 2002; Phillips-Portillo 2012). Hence, I assume that the lateral cluster could be the tangential lineage. In the larval brain, the lateral cluster apparently projected outside of the CB upper rim (Figure 4.18D-F: arrows), but this statement is based on indirect evidence. This assumption could be confirmed by performing anti-synapsin or phalloidin staining, which mark the neuropils. In the embryo, the axonal projection from the lateral cluster could also be seen but not as obvious as the tracts sent by the median cluster (Figure 4.16G: orange dashed circle). They finally joined the axonal tracts sent from the median cluster and contributed to the central brain commissures.

Based on the contributions of the lateral cluster and the median cluster in the larval and adult brain, I could make a statement that the brain commissures marked by $T c$-foxQ2up line in the embryo are the $C X$ primodium, thereby revealing that Tc-FoxQ2 lineages contribute to the CX development. Indeed, in Schistocerca 
the $C B$ forms from commissural structures (Boyan et al., 2008b).

\subsubsection{Tc-FoxQ2 lineages- type I or type II}

It is known from Drosophila that type I NBs contribute to the small and diffused neuropils and MBs in the anterior brain, whereas type II NBs generate large neural lineages contributing to the CX (Bello et al., 2008; Izergina et al., 2009). In Drosophila, eight type II NBs have been identified to contribute to the CX development (Bello et al., 2008; Izergina et al., 2009; Walsh and Doe, 2017). Two of them are located more laterally, and six of them called DM1-6 NBs are located in the dorsomedial (DM) region generating remarkably large lineages. In Schistocerca, the identified four $W, X, Y, Z$ lineages contributing to the $C X$ were also shown to be type II lineages (Boyan and Williams 1997; Williams et al., 2005; Williams and Boyan 2008; Boyan et al., 2010). In this study, I identified two Tc-FoxQ2 neural lineages contributing to the $C X$ development. The FAM2 NB was located in the pars intercerebralis giving rise to a median columnar lineage and the A-PD NB was located in the lateral area producing a lateral lineage. Interestingly, these two neural lineages are not remarkably large lineages because of the small number of the cells. The median lineage contained $\sim 65$ cells, while the lateral lineage contained $\sim 15$ cells. It has been shown that type II lineages contain on average 450 cells, whereas type I lineages contain around 100 to 150 cells (Williams et al., 2005; Bello et al., 2008; Williams and Boyan 2008; Izergina et al., 2009; Boyan et al., 2010; Pereanu et al., 2010; Riebli et al., 2013). In addition, in Drosophila, type I NBs are characterized by the transcription factors Dpn and Ase, and by the differentiation factor Pros which is expressed in the cytoplasm. Type II NBs are identified by Dpn expression, but not Ase or Pros expression (Zhu et al., 2011; Weng and Cohen, 2015; Xie et al., 2016). In this work I used Tc-ase as a NB marker to identify the Tc-FoxQ2 ${ }^{+}$NBs. However, Tc-dpn showed an unexpected expression in Tribolium (Marita Buescher, personal communication). In the conclusion, I hypothesize that Tc-FoxQ2 lineages might be type I lineages. Since the NBs in Tribolium brain have not been characterized, it is still difficult to know whether the Tc-foxQ2up line marks the type I or type II lineage.

\subsubsection{Transcription factors are co-expressed in the Tc-FoxQ2+}

\section{neuroblasts}

In the Drosophlia embryo, the brain NBs delaminate continuously and generate in a reproducible pattern. The identity of each NB is believed to be specified by the combination of transcription factors (Urbach and Technau, 2003; Steinmetz et al., 2010). However, in these studies several genes conserved in the anterior region of 
animals had not been included. The identification of Tc-FoxQ2 ${ }^{+} \mathrm{NBs}$ during the embryogenesis showed that such genes have to be considered. I showed that the number of Tc-FoxQ2 ${ }^{+}$NBs reduced over time (Figure 4.12). Further, I determined co-expression of other transcription factors in these Tc-FoxQ2 ${ }^{+} \mathrm{NBs}$.

\subsubsection{Tc-six 3 is expressed in the FAM2 neuroblast and A-PD}

\section{neuroblast}

The results showed that all Tc-FoxQ2 ${ }^{+} \mathrm{NBs}$ in the anterior neuroectoderm were Tc-six3 positive from NS8 to NS11 (Figure 4.13A-A'; Figure 4.14A-A'). From NS8 to NS14, it was shown that Tc-foxQ2 expression was the subset of $T c$-six 3 expression in the anterior neuroectoderm (Kitzmann et al., 2017). Hence, it is suggested that the FAM2 NB which generates the median columnar lineage and the A-PD NB which generates the lateral lineage are $T c$-six 3 positive. Furthermore, the brain phenotype of Tc-foxQ2 knock-down and Tc-six3 knock-down in L1 larvae shared some features, e.g. the defects in the CB (Posnien et al., 2011b; Kitzmann et al., 2017), which suggests a correlation of $T c-f o x Q 2$ and $T c$-six 3 in neural development. Therefore, it is suggested that Tc-FoxQ2 and Tc-six3 together (maybe in concert with other factors) specify the FAM2 NB and A-PD NB contributing to the CX development.

\subsubsection{Tc-chx is expressed in the FAM2 neuroblast}

It is known that four NBs in each hemisphere, which are located in the pars intercerebralis, contribute to the CX development in grasshopper Schistocerca. These NBs give rise to the stereotypic neural lineages which send the axon fascicles initially to the $P B$ and then to $C B$ via four discrete tracts $(W, X, Y, Z$ ) (Boyan and Williams, 1997; Williams et al., 2005; Williams and Boyan, 2008; Boyan et al., 2008; Boyan and Reichert, 2011). In Drosophila, a similar set of four NBs in each hemisphere were also observed, four columnar lineages (DM1-4) which corresponded to the $W, X, Y, Z$ lineages in grasshopper contributing to the $C X$ (Izergina et al., 2009; Young and Armstrong 2010; Boyan and Reichert 2011). Further, $D c h \times 1$ is known to be specifically expressed in the pars intercerebralis (de Velasco et al., 2007). In Tribolium, the expression of Tc-chx was also found in the pars intercerebralis. Moreover, the expression of Tc-chx was observed to be overlapping with Tc-foxQ2 in the pars intercerebralis (Posnien et al., 2011b; Kitzmann et al., 2017). Further, Tc-chx was shown to play an important role in the CB formation. Tc-chx knock-down led to the loss of the CB (Koniszewski, 2011). One Tc-FoxQ2 ${ }^{+} \mathrm{NB}$ in the anterior median region, which probably corresponds to the pars intercerebralis, were the $T \mathrm{c}$ - $c h x$ positive (Figure $4.14 \mathrm{C}-\mathrm{C}^{\prime}$ ) and contribute to the CX. As the four NBs identified in Drosophila and Schistocerca are type II NBs, it 80 
is hypothesized that the FAM2 NB is not a homologue of these four NBs. It could be an additional NB contributing to the $C X$.

\subsubsection{Tc-rx and Tc-ey might play a role in specifying the central complex neuroblast}

The identified NBs contributing to the $C X$ are located in the pars intercerebralis of Schistocera, as well as in Drosophila (Boyan and Reichert, 2011). Previous studies in Tribolium suggested that Tc-rx has no connection with these NBs because $T c-r x$ is not expressed in the pars intercerebralis (Koniszewski, 2011). Instead, Tc-rx was shown to be expressed in the lateral neurogenic region (Posnien et al., 2011b; Kitzmann et al., 2017). However, it was shown that Tc-rx knock-down resulted in a split CB in the L1 larval brain (Koniszewski, 2011). This suggested that Tc-rx was probably involved in the formation of the $C B$. This is in line with the findings in Drsophila where $r x$ is required for development of the ellipsoid body (EB) (Davis et al., 2003), which is part of the CX. However, it remained unclear, what neural lineage was affected in these $r x$ knock-down situations. Based on my findings I can hypothesize what NBs might be involved. In this work, I found that Tc-rx was expressed in the A-PD NB which was located at the anterolateral region of the brain and not in the pars intercerebralis from NS8 to NS11 (Figure 4.13D-D'; Figure 4.14D-D'). Hence, $T c-r x$ might play a role in specifying the A-PD NB that forms the lateral lineage, which contributes to the $\mathrm{CX}$.

I also found that Tc-ey was expressed in the Tc-rx/FoxQ2 ${ }^{+}$A-PD NB from NS8 to NS11 (Figure 4.13E-E'; Figure 4.14E-E'). Previous studies suggested that Tc-ey marks MB NBs since the expression domain of Tc-ey in the anterior neuroectoderm of Tribolium was similar to the domain in Drosophila where the MB NBs arise (Posnien et al., 2011b). Moreover, ey has been shown to be a key regulator of $M B$ development in Drosophila (Kurusu et al., 2000, Noveen et al., 2000; Kunz et al., 2012). Hence, it appears that the A-PD NB determined here could be one of the MB NBs but unexpectedly, it also contributed to the CX. It still needs to be clarified whether Tc-ey is required for CX contribution of the A-PD NB.

In summary, Tc-FoxQ2, Tc-six3 and Tc-chx that are co-expressed in the FAM2 NB in the pars intercerebralis together probably specify the FAM2 NB, which forms the median columnar lineage contributing to the $C X$ in Tribolium. An additional A-PD $\mathrm{NB}$, which is located in the anterolateral region, does not belong to the pars intercerebralis, but generates the lateral lineage contributing to the $C X$ development. This A-PD NB co-expresses Tc-FoxQ2, Tc-six3, possibly Tc-rx and Tc-ey. 


\subsection{Tc-foxQ2 is involved in building the axon scaffold in}

\section{the central brain}

The protocerebrum is different from the segmental ganglia in several respects. Among other issues, the commissures crossing the midline are much more prominent in the protocerebrum and they contribute to midline-spanning neuropils, which are absent in the VNC. This difference needs to be reflected in cellular differences regulated by genes. Genes active in the anterior neuroectoderm but not in the more posterior CNS are prime candidates to be involved in shaping the difference. Hence, Tc-foxQ2 could be one of these genes. In order to study whether Tc-foxQ2 is involved in the axon scaffold formation in the embryo, Tc-foxQ2 knock-down was performed in $S B$ animal, combined with the immunostaining for ac. tubulin which is a general neuronal marker labeling the axons. The axonal bundles spanning the central brain were reduced and/or aberrant and the projections across the midline were not formed properly such that the commissures were affected after Tc-foxQ2 RNAi (Figure 5.2B, C). In the strongest phenotype, the brain commissures were even absent (Figure 5.3C'). This complete disorder or loss suggests that $T c$-foxQ2 is involved in building the axon scaffold in the central brain.

Do Tc-foxQ2 positive cells themselves contribute to the commissures? In Tc-foxQ2up line, EGFP positive cells located in the contralateral side projected through the initial commissure, but they did not pioneer the primary brain commissure (Figure 4.16B: arrowhead, white dashed line). This is based on the observation that when the primary brain commissure formed, no co-localization of ac. tubulin and EGFP was observed. The EGFP positive commissures were observed only later when the ac. tubulin marked commissures were already quite developed. However, I cannot exclude some delay of EGFP signal compared to the ac. tubulin signal because it might take some time until it diffuses into the axons and accumulates sufficient molecules to become detectable. In the adult, the $T c$-foxQ2 positive neurons significantly contributed to PB spanning the midline.

Taken together, functional tests and contribution of marked cells suggest that Tc-foxQ2 function indeed contributes to the formation of extensive midline structures in the protocerebrum. 


\subsection{The brain phenotype of $T c-f o x Q 2$ in the embryo}

It has been shown that $T c-f o x Q 2$ is required for brain development, leading to defects in the $C B$ and the $M B s$ in the $L 1$ larvae in Tc-foxQ2 knock-down experiments. The phenotype of $T c-f o x Q 2$ knock-down showed a reduced $C B$ and the fusion of the medial lobes of the MBs (Kitzmann et al., 2017). In other species, foxQ2 has also been shown to have anterior neural functions. In sea urchin Strongylocentrotus and sea anemone cnidarian Nematostella, foxQ2 knock-down led to the alteration in the structure of the neurosecretory apical organ (Sinigaglia et al., 2013; Yaguchi et al., 2012, 2010, 2008). In the centipede Strigamia, based on the expression pattern of foxQ2 in the apical plate, a neural function was also proposed (Hunnekuhl and Akam, 2014). One of the aims of this study was to get a more precise view on the $T c-f o x Q 2$ brain phenotype. What exactly happened to cell bodies and the axonal projections which contribute to the central brain after Tc-foxQ2 knock-down? To answer this question, I used different transgenic lines and antibodies which marked the central brain and described the Tc-foxQ2 knock-down phenotypes with these lines.

In the lines E011A-01 (a Tc-rx enhancer trap line from the GEKU screen, in which a construct containing EGFP under the control of a 3xP3 promoter is inserted) and $T c-r x$ 178, (a Tc-rx reporter line contains parts of the upstream genomic region of Tc-rx fused with basal promoter elements driving DsRedEx) (Koniszewski, 2011), Tc-foxQ2 knock-down showed that the marked cells contributing to the central brain commissures were almost gone and the number of the cells which contributed to the central brain commissures was significantly decreased (Figure 5.4B; Figure 5.5B). In the line E035004 (an enhancer trap line derived from the GEKU screen), the strongest phenotype revealed that the brain hemispheres were reduced and fused at the midline, and the brain commissures were completely absent (Figure 5.3C). The fact that the entire commissure is lacking (ac. tubulin staining) or disturbed (ac. tubulin and imaging lines) shows that Tc-foxQ2 cells do not just project across the midline but are required for its formation. These findings further confirm that Tc-foxQ2 has an early and central function in the formation of the brain commissures. It is likely that the cells or mechanisms guiding and forming the axon scaffold are affected after Tc-foxQ2 knock-down, which finally leads to a disordered or lacking axon scaffold. Therefore, Tc-foxQ2 RNAi could lead to reduction or loss of the central brain commissures, which could in turn result in the observed phenotype with respect to other cells usually projecting across the scaffolds.

In Tc-foxQ2up line, knock-down of Tc-foxQ2 showed a reduced number of cell bodies as well as their axonal projections (Figure 5.1B, D). The brain commissures which were shown to be in the CX primodium were not completely absent (Figure 5.1D: arrowhead). It is unclear, whether this reflects the strongest phenotype or 
whether residual Tc-foxQ2 function could have been present in these animals. In order to get better understanding of the loss-of-function phenotype, a Tc-foxQ2 mutant line should be generated, which is easily done using the CRISPR/Cas9 system (Garneau et al., 2010; Jinek et al., 2012; Gilles et al., 2015; Schwertner 2016, unpublished). By knocking in a reporter gene into the coding sequence of Tc-foxQ2, a loss of Tc-foxQ2 function would be generated and the insertion would be simultaneously visualized by the eye marker. Recent studies showed that in the zebrafish phenotypes from the transient knock-down experiments showed quite some difference from phenotypes from the stable loss-of-function mutant lines (Rossi et al., 2015). It would be interesting to see whether Tc-foxQ2 mutants show the completely loss of the CX primordium. Moreover, it would be interesting to analyze whether Tc-foxQ2 mutants have additional defects.

\subsection{The heterochrony in the central body development}

The timing of the $\mathrm{CB}$ development differs in insects. In most hemimetabolous, e.g. the orthopteran Schistocerca gregaria, the CB completely develops at the end of the embryogenesis, and just undergoes growth in size without major changes in morphology during postembryonic development (Boyan and Williams 1997, 2011; Williams et al., 2005; Boyan and Reichert 2011). In some species such as flies (Musca, Drosophila), butterflies (Pieris), moths (Ephestia) and bees (Apis), the CB develops relatively late and is undetectable at late embryonic stage (Panov, 1959). In Drosophila, the first identifiable CB appears in the third instar larva, showing the immature FB (Young and Armstrong 2010). These timing differences of development have been termed "heterochrony" (Gould, 1977; Koniszewski et al., 2016). The heterochronic development of the CB in different species may be related with the functions, which could be explained by the corresponding formation of walking legs. In Schistocerca the CB fully developed during embryogenesis and the hatched larvae have developed walking legs, whereas in Tribolium the hatchlings possess immature walking legs and in Drosophila the hatchlings do not have walking legs until become adults (Pfeiffer and Homberg 2014; Koniszewski et al., 2016).

It is known that in tenebrionid beetles, such as Tribolium and Tenebrio, the CB partially forms during the embryogenesis (Wegerhoff and Breidbach 1992; Wegerhoff et al., 1996; Koniszewski et al., 2016). In the L1 larval brain of Tribolium, the $\mathrm{FB}$, which is the upper unit of the $\mathrm{CB}$, was detected by using synapsin staining. The PB was also present in L1 larvae but it was split medially (Koniszewski et al., 2016). In this work, the CX primodium, which was marked by Tc-foxQ2up line, was first detected at stage NS14, showing the axonal bundles projecting towards the midline but not yet joining at the midline (Figure 4.16C: arrowhead). At the last embryonic stage, the CX primodium spanning the midline and connecting the two brain hemispheres was observed (Figure 4.16E: arrowhead). This study allows the 
visualization of the part of the $C X$ primodium which is built by the Tc-FoxQ2 positive cells in the embryonic stage, further providing the evidence for $C X$ development during embryogenesis. In the adult brain, the PB was fully developed, which was detected by using synapsin staining (Figure $4.19 C^{\prime}$ ). The $C B$ could be marked by the line E011A-01 (data not shown). Here, only the upper unit of the $C B$ was marked by Tc-foxQ2 up line in the adult brain (Figure 4.19D).

It is known that the Drosophila ortholog of foxQ2 is $f d 102 c$, which is expressed in a large number of neurons in the brain hemispheres at the late embryonic stage (Lee and Frasch, 2004). Hence, marking fd102c cells in Drosophila might help to monitor the differences of development.

\subsection{Outlook}

In this study, I used Tc-ase-RNA intronic probe to mark the NBs. Although it showed better resolution than Tc-ase-RNA exonic probe, I was not able to identify the Tc-FoxQ2 ${ }^{+}$NBs at the latest stage (NS15) due to the large number of the cells in the brain. Therefore, antibody staining is necessary for the cellular level study. Double immunostainings could provide more precise insights about the co-localization of the Tc-FoxQ2 and a NB marker (e.g. Tc-Ase or Tc-Dpn) and the number of Tc-FoxQ2 ${ }^{+}$NBs at later stages in the embryo. It would be also interesting to generate a transgenic line to mark Tc-ase with a reporter gene by using the CRISPR/Cas9 system. The line could be used in combination with the fluorescent marker protein together with the Tc-FoxQ2 antibody.

Previous study showed that NBs undergo quiescence at the embryo-larval transition in Drosophila (Truman and Bate, 1988). Thus, it would be worth investigating the Tc-FoxQ2 ${ }^{+} \mathrm{NBs}$. To this end, phospho-histone $\mathrm{H} 3(\mathrm{pH} 3)$ antibody staining which mark the mitotic cells could be done with the embryos at later stages and the newly hatched larvae. This could find out if only the Tc-FoxQ2 ${ }^{+} \mathrm{NB}$ divides or also additional cells close to the Tc-FoxQ2 ${ }^{+}$NB divide.

This work showed that Tc-foxQ2 knock-down results in a very small number of the brains showing the strongest phenotype, which is characterized by the loss of the brain commissures. The frequent phenotypes still showed the axonal projections across the midline, which seemed not to act on the top level. Thus, the following question arises: is this due to a low penetrance of RNAi treatment or a compensatory trait of the system? To answer this question, a loss of Tc-foxQ2 function should be studied. This could be achieved by generating a transgenic Tc-foxQ2-null mutant by using the CRISPR/Cas9. Knock-in of a marker into the coding sequence of $T c$-foxQ2 would lead to a loss of $T c$-foxQ2 function. Then these mutants could be used to analyze their brain phenotype. It should be analyzed 
whether the mutants show the strengthened phenotype, like the complete absence of the brain commissures and the reduced number of Tc-foxQ2 positive cells in the anterior brain. Further, it would be interesting whether Tc-foxQ2-null mutants show additional defects. Hence, a Tc-foxQ2-null mutant line could be used to test whether the brain phenotype is complete.

It would be also interesting to get more details on the brain defects in Tc-foxQ2 knock-down larvae. The dissected larval brains could be stained with the neural markers which mark the CX, like anti-synapsin, anti-5HT (serotonin), anti-myoinhibitory protein (MIP), anti-periviscerokinin (PVK), and anti- TKRP (tachykinin-related peptide) (Koniszewski et al., 2016).

The Tc-foxQ2 enhancer trap line also had the potential of further use as a Cre recombinase was integrated. Crossing this line with other loxP lines could mark the cells permanently with a different fluorescent protein. Additionally, gene ablations could be also possible among other Cre/loxP experiments. 


\section{References}

Armstrong, J. D., de Belle, J. S., Wang, Z. \& Kaiser, K. Metamorphosis of the mushroom bodies; large-scale rearrangements of the neural substrates for associative learning and memory in Drosophila. Learning \& Memory 5, 102-114 (1998).

Auer, T. O., Duroure, K., De Cian, A., Concordet, J.-P. \& Del Bene, F. Highly efficient CRISPR/Cas9-mediated knock-in in zebrafish by homology-independent DNA repair. Genome research 24, 142-153 (2014).

Awata, H., Watanabe, T., Hamanaka, Y., Mito, T., Noji, S., \& Mizunami, M. Knockout crickets for the study of learning and memory: Dopamine receptor Dop1 mediates aversive but not appetitive reinforcement in crickets. Scientific reports 5, 15885 (2015).

Bailey, T. J., El-Hodiri, H., Zhang, L., Shah, R., Mathers, E. H., \& Jamrich, M. Regulation of vertebrate eye development by Rx genes. International Journal of Developmental Biology 48, 761-770 (2004).

Bassett, A. R., Tibbit, C., Ponting, C. P. \& Liu, J. L. Highly efficient targeted mutagenesis of Drosophila with the CRISPR/Cas9 system. Cell reports 4, 220-228 (2013).

Bayraktar, O. A., Boone, J. Q., Drummond, M. L. \& Doe, C. Q. Drosophila type II neuroblast lineages keep Prospero levels low to generate large clones that contribute to the adult brain central complex. Neural development 5, 26 (2010).

Beatus, P. \& Lendahl, U. Notch and neurogenesis. Journal of neuroscience research 54, 125-136 (1998).

Bello, B. C., Izergina, N., Caussinus, E. \& Reichert, H. Amplification of neural stem cell proliferation by intermediate progenitor cells in Drosophila brain development. Neural development 3, 5 (2008).

Benayoun, B. A., Caburet, S. \& Veitia, R. A. Forkhead transcription factors: key players in health and disease. Trends in Genetics 27, 224-232 (2011).

Berghammer, A. J., Klingler, M. \& Wimmer, E. A. Genetic techniques: a universal marker for transgenic insects. Nature 402, 370 (1999).

Bernhardt, R. R., Chitnis, A. B., Lindamer, L. \& Kuwada, J. Y. Identification of spinal neurons in the embryonic and larval zebrafish. Journal of comparative neurology 302, 603-616 (1990).

Bhat, K. M. Segment polarity genes in neuroblast formation and identity specification during Drosophila neurogenesis. Bioessays 21, 472-485 (1999).

Bi, H. L., Xu, J., Tan, A. J. \& Huang, Y. P. CRISPR/Cas9-mediated targeted gene mutagenesis 
in Spodoptera litura. Insect science 23, 469-477 (2016).

Biffar, L. Early neurogenesis in the flour beetle Tribolium castaneum, Queen Mary University of London, (2013).

Black, M. M. \& Keyser, P. Acetylation of alpha-tubulin in cultured neurons and the induction of alpha-tubulin acetylation in PC12 cells by treatment with nerve growth factor. Journal of Neuroscience 7, 1833-1842 (1987).

Boone, J. Q. \& Doe, C. Q. Identification of Drosophila type II neuroblast lineages containing transit amplifying ganglion mother cells. Developmental neurobiology 68, 1185-1195 (2008).

Bossing, T., Udolph, G., Doe, C. Q. \& Technau, G. M. The Embryonic Central Nervous System Lineages of Drosophila melanogaster: I. Neuroblast Lineages Derived from the Ventral Half of the Neuroectoderm. Developmental biology 179, 41-64 (1996).

Boyan, G. \& Liu, Y. Timelines in the insect brain: fates of identified neural stem cells generating the central complex in the grasshopper Schistocerca gregaria. Development genes and evolution 224, 37-51 (2014).

Boyan, G., Therianos, S., Williams, J. \& Reichert, H. Axogenesis in the embryonic brain of the grasshopper Schistocerca gregaria: an identified cell analysis of early brain development. Development 121, 75-86 (1995a).

Boyan, G. \& Williams, J. Embryonic development of the pars intercerebralis/central complex of the grasshopper. Development genes and evolution 207, 317-329 (1997).

Boyan, G., Williams, J. \& Herbert, Z. Fascicle switching generates a chiasmal neuroarchitecture in the embryonic central body of the grasshopper Schistocerca gregaria. Arthropod structure \& development 37, 539-544 (2008).

Boyan, G., Williams, J. \& Herbert, Z. An ontogenetic analysis of locustatachykinin-like expression in the central complex of the grasshopper Schistocerca gregaria. Arthropod structure \& development 37, 480-491 (2008).

Boyan, G. \& Williams, L. Embryonic development of the insect central complex: insights from lineages in the grasshopper and Drosophila. Arthropod structure \& development 40, 334-348 (2011).

Boyan, G., Williams, L., Legl, A. \& Herbert, Z. Proliferative cell types in embryonic lineages of the central complex of the grasshopper Schistocerca gregaria. Cell and tissue research 341, 259-277 (2010).

Boyan, G., Williams, L. \& Meier, T. Organization of the commissural fibers in the adult brain of the locust. Journal of Comparative Neurology 332, 358-377 (1993).

Boyan, G. S. \& Reichert, H. Mechanisms for complexity in the brain: generating the insect central complex. Trends in neurosciences 34, 247-257 (2011).

Boyan, G. S., Williams, J. L. D. \& Reichert, H. Morphogenetic reorganization of the brain 
during embryogenesis in the grasshopper. Journal of comparative neurology 361, 429-440 (1995b).

Boyan, G. S., Williams, J. L. D. \& Reichert, H. Organization of a midline proliferative cluster in the embryonic brain of the grasshopper. Roux's archives of developmental biology 205, 45-53 (1995c).

Brand, M., Jarman, A. P., Jan, L. Y. \& Jan, Y. N. asense is a Drosophila neural precursor gene and is capable of initiating sense organ formation. Development 119, 1-17 (1993).

Brandt, R., Rohlfing, T., Rybak, J., Krofczik, S., Maye, A., Westerhoff, M., Hege H. \& Menzel, R. Three-dimensional average-shape atlas of the honeybee brain and its applications. Journal of Comparative Neurology 492, 1-19 (2005).

Brown, S. J., Mahaffey, J. P., Lorenzen, M. D., Denell, R. E. \& Mahaffey, J. W. Using RNAi to investigate orthologous homeotic gene function during development of distantly related insects. Evolution \& development 1, 11-15 (1999).

Brown, S. J. Shippy, T. D., Miller, S., Bolognesi, R., Beeman, R. W., Lorenzen, M. D., Bucher, G., Wimmer E. A. \& Klingler, M. The red flour beetle, Tribolium castaneum (Coleoptera): a model for studies of development and pest biology. Cold Spring Harbor Protocols 2009, pdb. emo126 (2009).

Bucher, G., Scholten, J. \& Klingler, M. Parental RNAi in tribolium (coleoptera). Current Biology 12, R85-R86 (2002).

Cabrera, C. V., Martinez-Arias, A. \& Bate, M. The expression of three members of the achaete-scute gene complex correlates with neuroblast segregation in Drosophila. Cell 50, 425-433 (1987).

Campbell, G., Goring, H., Lin, T., Spana, E., Andersson, S., Doe, C. Q., \& Tomlinson, A. RK2, a glial-specific homeodomain protein required for embryonic nerve cord condensation and viability in Drosophila. Development 120, 2957-2966 (1994).

Campuzano, S., Carramolino, L., Cabrera, C. V., Ruiz-Gómez, M., Villares, R., Boronat, A., \& Modolell, J. Molecular genetics of the achaete-scute gene complex of D. melanogaster. Cell 40, 327-338 (1985).

Campuzano, S. \& Modolell, J. Patterning of the Drosophila nervous system: the achaete-scute gene complex. Trends in Genetics 8, 202-208 (1992).

Carroll, D. Genome engineering with targetable nucleases. Annual review of biochemistry 83, 409-439 (2014).

Cayre, M., Strambi, C., Charpin, P., Augier, R., Meyer, M. R., Edwards, J. S., \& Strambi, A. Neurogenesis in adult insect mushroom bodies. Journal of comparative neurology 371, 300-310 (1996).

Clark, I. B., Boyd, J., Hamilton, G., Finnegan, D. J. \& Jarman, A. P. D-six4 plays a key role in patterning cell identities deriving from the Drosophila mesoderm. Developmental biology 294, 220-231 (2006). 
D'Aniello, E., Pezzotti, M. R., Locascio, A. \& Branno, M. Onecut is a direct neural-specific transcriptional activator of $\mathrm{Rx}$ in Ciona intestinalis. Developmental biology 355, 358-371 (2011).

Davis, R. J., Tavsanli, B. C., Dittrich, C., Walldorf, U. \& Mardon, G. Drosophila retinal homeobox (drx) is not required for establishment of the visual system, but is required for brain and clypeus development. Developmental biology 259, 272-287 (2003).

De Velasco, B., Erclik, T., Shy, D., Sclafani, J., Lipshitz, H., Mclnnes, R., \& Hartenstein, V. Specification and development of the pars intercerebralis and pars lateralis, neuroendocrine command centers in the Drosophila brain. Developmental biology 302, 309-323 (2007).

Doe, C. Q. Molecular markers for identified neuroblasts and ganglion mother cells in the Drosophila central nervous system. Development 116, 855-863 (1992).

Doe, C. Q. Neural stem cells: balancing self-renewal with differentiation. Development 135 , 1575-1587 (2008).

Doe, C. Q. \& Goodman, C. S. Early events in insect neurogenesis: I. Development and segmental differences in the pattern of neuronal precursor cells. Developmental biology 111, 193-205 (1985).

Dong, Y. \& Friedrich, M. Nymphal RNAi: systemic RNAi mediated gene knockdown in juvenile grasshopper. BMC biotechnology 5, 25 (2005).

Dreyer, D., Vitt, H., Dippel, S., Goetz, B., El Jundi, B., Kollmann, M., Huetteroth W. \& Schachtner, J. 3D standard brain of the red flour beetle Tribolium castaneum: a tool to study metamorphic development and adult plasticity. Frontiers in Systems Neuroscience 4, 3 (2010).

Egger, B., Chell, J. M. \& Brand, A. H. Insights into neural stem cell biology from flies. Philosophical Transactions of the Royal Society of London B: Biological Sciences 363, 39-56 (2008).

Eggert, T., Hauck, B., Hildebrandt, N., Gehring, W. J. \& Walldorf, U. Isolation of a Drosophila homolog of the vertebrate homeobox gene $\mathrm{Rx}$ and its possible role in brain and eye development. Proceedings of the National Academy of Sciences 95, 2343-2348 (1998).

El-Sherif, E., Averof, M. \& Brown, S. J. A segmentation clock operating in blastoderm and germband stages of Tribolium development. Development, dev. 085126 (2012).

El Jundi, B., Heinze, S., Lenschow, C., Kurylas, A., Rohlfing, T., \& Homberg, U. The locust standard brain: a 3D standard of the central complex as a platform for neural network analysis. Frontiers in systems neuroscience 3, 21 (2010).

Elphick, M., Williams, L. \& Shea, M. New features of the locust optic lobe: evidence of a role for nitric oxide in insect vision. Journal of Experimental Biology 199, 2395-2407 (1996).

Erclik, T., Hartenstein, V., Lipshitz, H. D. \& Mclnnes, R. R. Conserved role of the Vsx genes 
supports a monophyletic origin for bilaterian visual systems. Current Biology 18, 1278-1287 (2008).

Fascetti, N. \& Baumgartner, S. Expression of Drosophila Ten-a, a dimeric receptor during embryonic development. Mechanisms of development 114, 197-200 (2002).

Florentin, A. \& Arama, E. Caspase levels and execution efficiencies determine the apoptotic potential of the cell. J Cell Biol 196, 513-527 (2012).

Garneau, J. E., Dupuis, M. Ė., Villion, M., Romero, D. A., Barrangou, R., Boyaval, P., Fremaux, C., Horvath, P., Magadán, A. H. \& Moineau S. The CRISPR/Cas bacterial immune system cleaves bacteriophage and plasmid DNA. Nature 468, 67 (2010).

Gasiunas, G., Barrangou, R., Horvath, P. \& Siksnys, V. Cas9-crRNA ribonucleoprotein complex mediates specific DNA cleavage for adaptive immunity in bacteria. Proceedings of the National Academy of Sciences 109, E2579-E2586 (2012).

Gilles, A. F. \& Averof, M. Functional genetics for all: engineered nucleases, CRISPR and the gene editing revolution. EvoDevo 5, 43 (2014).

Gilles, A. F., Schinko, J. B. \& Averof, M. Efficient CRISPR-mediated gene targeting and transgene replacement in the beetle Tribolium castaneum. Development 142, 2832-2839 (2015).

Gratz, S. J., Wildonger, J., Harrison, M. M. \& O'Connor-Giles, K. M. CRISPR/Cas9-mediated genome engineering and the promise of designer flies on demand. Fly 7, 249-255 (2013).

Grimaldi, D. \& Engel, M. S. Evolution of the Insects. (Cambridge University Press, 2005).

Hammond, A., Galizi, R., Kyrou, K., Simoni, A., Siniscalchi, C., Katsanos, D., et al. A CRISPR-Cas9 gene drive system targeting female reproduction in the malaria mosquito vector Anopheles gambiae. Nature biotechnology 34, 78 (2016).

Hanesch, U., Fischbach, K.-F. \& Heisenberg, M. Neuronal architecture of the central complex in Drosophila melanogaster. Cell and Tissue Research 257, 343-366 (1989).

Hartenstein, V. \& Campos-Ortega, J. A. Early neurogenesis in wild-type Drosophila melanogaster. Wilhelm Roux's archives of developmental biology 193, 308-325 (1984).

Hartenstein, V., Spindler, S., Pereanu, W. \& Fung, S. Brain development in Drosophila melanogaster 1-31 (2008).

Harzsch, S., Anger, K. \& Dawirs, R. Immunocytochemical detection of acetylated alpha-tubulin and Drosophila synapsin in the embryonic crustacean nervous system. International Journal of Developmental Biology 41, 477-484 (2002).

Hashimoto, H., Yabe, T., Hirata, T., Shimizu, T., Bae, Y. K., Yamanaka, Y., Hirano Toshio \& Hibi, M. Expression of the zinc finger gene fez-like in zebrafish forebrain. Mechanisms of development 97, 191-195 (2000).

Heinze, S., Florman, J., Asokaraj, S., El Jundi, B. \& Reppert, S. M. Anatomical basis of sun compass navigation II: the neuronal composition of the central complex of the monarch 
butterfly. Journal of Comparative Neurology 521, 267-298 (2013).

Heitzler, P., Bourouis, M., Ruel, L., Carteret, C. \& Simpson, P. Genes of the Enhancer of split and achaete-scute complexes are required for a regulatory loop between Notch and Delta during lateral signalling in Drosophila. Development 122, 161-171 (1996).

Hirata, T., Nakazawa, M., Muraoka, O., Nakayama, R., Suda, Y., \& Hibi, M. Zinc-finger genes Fez and Fez-like function in the establishment of diencephalon subdivisions. Development 133, 3993-4004 (2006).

Homberg, U. Interneurones of the central complex in the bee brain (Apis mellifera, L.). Journal of insect physiology 31, 251-264 (1985).

Homberg, U. Evolution of the central complex in the arthropod brain with respect to the visual system. Arthropod structure \& development 37, 347-362 (2008).

Homberg, U., Heinze, S., Pfeiffer, K., Kinoshita, M. \& El Jundi, B. Central neural coding of sky polarization in insects. Philosophical Transactions of the Royal Society of London B: Biological Sciences 366, 680-687 (2011).

Homem, C. C. \& Knoblich, J. A. Drosophila neuroblasts: a model for stem cell biology. Development 139, 4297-4310 (2012).

Horvath, P. \& Barrangou, R. CRISPR/Cas, the immune system of bacteria and archaea. Science 327, 167-170 (2010).

Hunnekuhl, V. S. \& Akam, M. An anterior medial cell population with an apical-organ-like transcriptional profile that pioneers the central nervous system in the centipede Strigamia maritima. Developmental biology 396, 136-149 (2014).

Ito, K., Awano, W., Suzuki, K., Hiromi, Y. \& Yamamoto, D. The Drosophila mushroom body is a quadruple structure of clonal units each of which contains a virtually identical set of neurones and glial cells. Development 124, 761-771 (1997).

Ito, K. \& Hotta, Y. Proliferation pattern of postembryonic neuroblasts in the brain of Drosophila melanogaster. Developmental biology 149, 134-148 (1992).

Izergina, N., Balmer, J., Bello, B. \& Reichert, H. Postembryonic development of transit amplifying neuroblast lineages in the Drosophila brain. Neural development 4, 44 (2009).

Jefferis, G. S., Potter, C. J., Chan, A. M., Marin, E. C., Rohlfing, T., Maurer, C. R., \& Luo, L.Comprehensive maps of Drosophila higher olfactory centers: spatially segregated fruit and pheromone representation. Cell 128, 1187-1203 (2007).

Jiang, W., Bikard, D., Cox, D., Zhang, F. \& Marraffini, L. A. RNA-guided editing of bacterial genomes using CRISPR-Cas systems. Nature biotechnology 31, 233 (2013).

Jinek, M., Chylinski, K., Fonfara, I., Hauer, M., Doudna, J. A., \& Charpentier, E. A programmable dual-RNA-guided DNA endonuclease in adaptive bacterial immunity. Science, 1225829 (2012).

Kaiser, A. Immuncytochemische Färbungen und 3D-Rekonstruktionen am Zentralkomplex 92 
der Westlichen Honigbiene Apis mellifera, (2014).

Karlsson, D., Baumgardt, M. \& Thor, S. Segment-specific neuronal subtype specification by the integration of anteroposterior and temporal cues. PLoS biology 8, e1000368 (2010).

Kaufmann, E. \& Knöchel, W. Five years on the wings of fork head. Mechanisms of development 57, 3-20 (1996).

Kittelmann, S. Formation of the Clypeolabral Region During Embryonic Head Development of the Red Flour Beetle Tribolium castaneum, Niedersächsische Staats-und Universitätsbibliothek Göttingen, (2012).

Kittelmann, S., Ulrich, J., Posnien, N. \& Bucher, G. Changes in anterior head patterning underlie the evolution of long germ embryogenesis. Developmental biology 374, 174-184 (2013).

Kitzmann, P., Weißkopf, M., Schacht, M. I. \& Bucher, G. foxQ2 has a key role in anterior head and central brain patterning in insects. Development, dev. 147637 (2017).

Knoblich, J. A. Mechanisms of asymmetric stem cell division. Cell 132, 583-597 (2008).

Koniszewski, N. Functional analysis of embryonic brain development in Tribolium castaneum. (2011).

Koniszewski, N. D. B., Kollmann, M., Bigham, M., Farnworth, M., He, B., Büscher, M., et al. The insect central complex as model for heterochronic brain development-background, concepts, and tools. Development genes and evolution 226, 209-219 (2016).

Kraft, K. F., Massey, E. M., Kolb, D., Walldorf, U. \& Urbach, R. Retinal homeobox promotes cell growth, proliferation and survival of mushroom body neuroblasts in the Drosophila brain. Mechanisms of development 142, 50-61 (2016).

Kume, T., Jiang, H., Topczewska, J. M. \& Hogan, B. L. The murine winged helix transcription factors, Foxc1 and Foxc2, are both required for cardiovascular development and somitogenesis. Genes \& development 15, 2470-2482 (2001).

Kunz, T., Kraft, K. F., Technau, G. M. \& Urbach, R. Origin of Drosophila mushroom body neuroblasts and generation of divergent embryonic lineages. Development, dev. 077883 (2012).

Kurusu, M., Awasaki, T., Masuda-Nakagawa, L. M., Kawauchi, H., Ito, K., \& Furukubo-Tokunaga, K. Embryonic and larval development of the Drosophila mushroom bodies: concentric layer subdivisions and the role of fasciclin II. Development 129, 409-419 (2002).

Kurusu, M., Nagao, T., Walldorf, U., Flister, S., Gehring, W. J., \& Furukubo-Tokunaga, K. Genetic control of development of the mushroom bodies, the associative learning centers in the Drosophila brain, by the eyeless, twin of eyeless, and Dachshund genes. Proceedings of the National Academy of Sciences 97, 2140-2144 (2000).

Kurylas, A. E., Rohlfing, T., Krofczik, S., Jenett, A. \& Homberg, U. Standardized atlas of the 
brain of the desert locust, Schistocerca gregaria. Cell and tissue research 333, 125 (2008).

Larsen, C., Shy, D., Spindler, S. R., Fung, S., Pereanu, W., Younossi-Hartenstein, A., \& Hartenstein, V. Patterns of growth, axonal extension and axonal arborization of neuronal lineages in the developing Drosophila brain. Developmental biology 335, 289-304 (2009).

Lee, B. P. \& Jones, B. W. Transcriptional regulation of the Drosophila glial gene repo. Mechanisms of development 122, 849-862 (2005).

Lee, H. H. \& Frasch, M. Survey of forkhead domain encoding genes in the Drosophila genome: classification and embryonic expression patterns. Developmental dynamics: an official publication of the American Association of Anatomists 229, 357-366 (2004).

Lee, T. Wiring the Drosophila Brain with Individually Tailored Neural Lineages. Current Biology 27, R77-R82 (2017).

Li, S.-J. \& Hochstrasser, M. A new protease required for cell-cycle progression in yeast. Nature 398, 246 (1999).

Li, Y., Zhang, J., Chen, D., Yang, P., Jiang, F., Wang, X., \& Kang, L.. CRISPR/Cas9 in locusts: Successful establishment of an olfactory deficiency line by targeting the mutagenesis of an odorant receptor co-receptor (Orco). Insect biochemistry and molecular biology 79, 27-35 (2016).

Loesel, R., Nässel, D. R. \& Strausfeld, N. J. Common design in a unique midline neuropil in the brains of arthropods. Arthropod Structure \& Development 31, 77-91 (2002).

Lorenzen, M. D., Brown, S. J., Denell, R. E. \& Beeman, R. W. Cloning and characterization of the Tribolium castaneum eye-color genes encoding tryptophan oxygenase and kynurenine 3-monooxygenase. Genetics 160, 225-234 (2002).

Mahlapuu, M., Ormestad, M., Enerback, S. \& Carlsson, P. The forkhead transcription factor Foxf1 is required for differentiation of extra-embryonic and lateral plate mesoderm. Development 128, 155-166 (2001).

Marin, E. C., Jefferis, G. S., Komiyama, T., Zhu, H. \& Luo, L. Representation of the glomerular olfactory map in the Drosophila brain. Cell 109, 243-255 (2002).

Mathers, P., Grinberg, A., Mahon, K. \& Jamrich, M. The Rx homeobox gene is essential for vertebrate eye development. Nature 387, 603 (1997).

Maurange, C., Cheng, L. \& Gould, A. P. Temporal transcription factors and their targets schedule the end of neural proliferation in Drosophila. Cell 133, 891-902 (2008).

McGinnis, W. \& Krumlauf, R. Homeobox genes and axial patterning. Cell 68, 283-302 (1992).

McNamara, K. J. A guide to the nomenclature of heterochrony. Journal of Paleontology 60 , 4-13 (1986). 
Mossessova, E. \& Lima, C. D. Ulp1-SUMO crystal structure and genetic analysis reveal conserved interactions and a regulatory element essential for cell growth in yeast. Molecular cell 5, 865-876 (2000).

Nériec, N. \& Desplan, C. From the eye to the brain: development of the Drosophila visual system. Current topics in developmental biology 116, 247-271 (2016).

Noveen, A., Daniel, A. \& Hartenstein, V. Early development of the Drosophila mushroom body: the roles of eyeless and dachshund. Development 127, 3475-3488 (2000).

Oberhofer, G., Grossmann, D., Siemanowski, J. L., Beissbarth, T. \& Bucher, G. $W n t / \beta$-catenin signaling integrates patterning and metabolism of the insect growth zone. Development, dev. 112797 (2014).

Oh, S. W., Harris, J. A., Ng, L., Winslow, B., Cain, N., Mihalas, S., Wang, Q., Lau, C., Kuan, L., Henry, A., et al. A mesoscale connectome of the mouse brain. Nature 508, 207 (2014).

Overton, P. M., Meadows, L. A., Urban, J. \& Russell, S. Evidence for differential and redundant function of the Sox genes Dichaete and SoxN during CNS development in Drosophila. Development 129, 4219-4228 (2002).

Panov, A. Structure of the insect brain at successive stages of postembryonic development. II. The central body. Entomol Rev 38, 276-283 (1959).

Pereanu, W., Kumar, A., Jennett, A., Reichert, H. \& Hartenstein, V. Development-based compartmentalization of the Drosophila central brain. Journal of Comparative Neurology 518, 2996-3023 (2010).

Pereanu, W., Younossi-Hartenstein, A., Lovick, J., Spindler, S. \& Hartenstein, V. Lineage-based analysis of the development of the central complex of the drosophila brain. Journal of Comparative Neurology 519, 661-689 (2011).

Pfeiffer, K. \& Homberg, U. Organization and functional roles of the central complex in the insect brain. Annual review of entomology 59, 165-184 (2014).

Phillips-Portillo, J. The central complex of the flesh fly, Neobellieria bullata: Recordings and morphologies of protocerebral inputs and small-field neurons. Journal of Comparative Neurology 520, 3088-3104 (2012).

Port, F., Chen, H.-M., Lee, T. \& Bullock, S. L. Optimized CRISPR/Cas tools for efficient germline and somatic genome engineering in Drosophila. Proceedings of the National Academy of Sciences 111, E2967-E2976 (2014).

Posnien, N., Koniszewski, N. \& Bucher, G. Insect Tc-six4 marks a unit with similarity to vertebrate placodes. Developmental biology 350, 208-216 (2011a).

Posnien, N., Koniszewski, N. D. B., Hein, H. J. \& Bucher, G. Candidate gene screen in the red flour beetle Tribolium reveals six 3 as ancient regulator of anterior median head and central complex development. PLoS genetics 7, e1002416 (2011b). 
Prokop, A. \& Technau, G. M. The origin of postembryonic neuroblasts in the ventral nerve cord of Drosophila melanogaster. Development 111, $79-88$ (1991).

Prokop, A. \& Technau, G. M. Normal function of the mushroom body defect gene of Drosophila is required for the regulation of the number and proliferation of neuroblasts. Developmental biology 161, 321-337 (1994).

Range, R. C. \& Wei, Z. An anterior signaling center patterns and sizes the anterior neuroectoderm of the sea urchin embryo. Development, dev. 128165 (2016).

Reichert, H. Drosophila neural stem cells: cell cycle control of self-renewal, differentiation, and termination in brain development. Cell Cycle in Development. Springer 529-546 (2011).

Reichert H, Boyan G. Building a brain: developmental insights in insects. Trends in neurosciences 20, 258-264 (1997).

Rein, K., Zöckler, M., Mader, M. T., Grübel, C. \& Heisenberg, M. The Drosophila standard brain. Current Biology 12, 227-231 (2002).

Ren, X., Sun, J., Housden, B. E., Hu, Y., Roesel, C., Lin, S., et al. Optimized gene editing technology for Drosophila melanogaster using germ line-specific Cas9. Proceedings of the National Academy of Sciences 110, 19012-19017 (2013).

Ren, X., Yang, Z., Xu, J., Sun, J., Mao, D., Hu, Y., et al. Enhanced specificity and efficiency of the CRISPR/Cas9 system with optimized sgRNA parameters in Drosophila. Cell reports $\mathbf{9}$, 1151-1162 (2014).

Richards, S., Gibbs, R. A., Weinstock, G. M., Brown, S. J., Denell, R., Beeman, R. W., Gibbs, R. et al. The genome of the model beetle and pest Tribolium castaneum. Nature 452, 949-955 (2008).

Riebli, N., Viktorin, G. \& Reichert, H. Early-born neurons in type II neuroblast lineages establish a larval primordium and integrate into adult circuitry during central complex development in Drosophila. Neural development 8, 6 (2013).

Rossi, A., Kontarakis, Z., Gerri, C., Nolte, H., Hölper, S., Krüger, M., \& Stainier, D. Y. Genetic compensation induced by deleterious mutations but not gene knockdowns. Nature 524, 230 (2015).

Rybak, J., Kuß, A., Hans, L., Zachow, S., Hege, H. C., Lienhard, M., Singer J., Neubert K. \& Menzel, R. The digital bee brain: integrating and managing neurons in a common $3 \mathrm{D}$ reference system. Frontiers in systems neuroscience 4, 30 (2010).

Saitoh, H., Pu, R. T. \& Dasso, M. SUMO-1: wrestling with a new ubiquitin-related modifier. Trends in biochemical sciences 22, 374-376 (1997).

Sander, J. D. \& Joung, J. K. CRISPR-Cas systems for editing, regulating and targeting genomes. Nature biotechnology 32, 347 (2014). 
Santagata, S., Resh, C., Hejnol, A., Martindale, M. Q. \& Passamaneck, Y. J. Development of the larval anterior neurogenic domains of Terebratalia transversa (Brachiopoda) provides insights into the diversification of larval apical organs and the spiralian nervous system. Evodevo 3, 3 (2012).

Sarrazin, A. F., Peel, A. D. \& Averof, M. A segmentation clock with two-segment periodicity in insects. Science 336, 338-341 (2012).

Schinko, J. B., Hillebrand, K. \& Bucher, G. Heat shock-mediated misexpression of genes in the beetle Tribolium castaneum. Development genes and evolution 222, 287-298 (2012).

Schinko, J. B. et al. Functionality of the GAL4/UAS system in Tribolium requires the use of endogenous core promoters. BMC developmental biology 10, 53 (2010).

Schmid, A., Chiba, A. \& Doe, C. Q. Clonal analysis of Drosophila embryonic neuroblasts: neural cell types, axon projections and muscle targets. Development 126, 4653-4689 (1999).

Schmidt, H., Rickert, C., Bossing, T., Vef, O., Urban, J., \& Technau, G. M. The embryonic central nervous system lineages of Drosophila melanogaster. Developmental biology 189, 186-204 (1997).

Schmitt-Engel, C., Schultheis, D., Schwirz, J., Ströhlein, N., Troelenberg, N., Majumdar, U., et al. The iBeetle large-scale RNAi screen reveals gene functions for insect development and physiology. Nature communications 6, 7822 (2015).

Schneider, L. E., Sun, E. T., Garland, D. J. \& Taghert, P. H. An immunocytochemical study of the FMRF amide neuropeptide gene products in Drosophila. Journal of Comparative Neurology 337, 446-460 (1993).

Sebo, Z. L., Lee, H. B., Peng, Y. \& Guo, Y. A simplified and efficient germline-specific CRISPR/Cas9 system for Drosophila genomic engineering. Fly 8, 52-57 (2014).

Seimiya, M. \& Gehring, W. J. The Drosophila homeobox gene optix is capable of inducing ectopic eyes by an eyeless-independent mechanism. Development 127, 1879-1886 (2000).

Sinigaglia, C., Busengdal, H., Leclere, L., Technau, U. \& Rentzsch, F. The bilaterian head patterning gene six $3 / 6$ controls aboral domain development in a cnidarian. PLoS biology 11, e1001488 (2013).

Skeath, J. B. At the nexus between pattern formation and cell-type specification: the generation of individual neuroblast fates in the Drosophila embryonic central nervous system. Bioessays 21, 922-931 (1999).

Skeath, J. B. \& Carroll, S. B. Regulation of proneural gene expression and cell fate during neuroblast segregation in the Drosophila embryo. Development 114, 939-946 (1992).

Skeath, J. B., Panganiban, G., Selegue, J. \& Carroll, S. Gene regulation in two dimensions: the proneural achaete and scute genes are controlled by combinations of axis-patterning genes through a common intergenic control region. Genes \& Development 6, 2606-2619 (1992). 
Steinmetz, P. R., Urbach, R., Posnien, N., Eriksson, J., Kostyuchenko, R. P., Brena, C., Guy, K., Akam, M., Bucher, G. \& Arendt, D. Six3 demarcates the anterior-most developing brain region in bilaterian animals. EvoDevo 1, 14 (2010).

Stocker, R., Lienhard, M., Borst, A. \& Fischbach, K. Neuronal architecture of the antennal lobe in Drosophila melanogaster. Cell and tissue research 262, 9-34 (1990).

Strausfeld, N. J., Sinakevitch, I., Brown, S. M. \& Farris, S. M. Ground plan of the insect mushroom body: functional and evolutionary implications. Journal of Comparative Neurology 513, 265-291 (2009).

Strausfeld, N. J., Sinakevitch, I. \& Vilinsky, I. The mushroom bodies of Drosophila melanogaster: an immunocytological and golgi study of Kenyon cell organization in the calyces and lobes. Microscopy research and technique 62, 151-169 (2003).

Strauss, R. The central complex and the genetic dissection of locomotor behaviour. Current opinion in neurobiology 12, 633-638 (2002).

Strobl, F., Schmitz, A. \& Stelzer, E. H. Live imaging of Tribolium castaneum embryonic development using light-sheet-based fluorescence microscopy. Nature protocols 10, 1486 (2015).

Strobl, F. \& Stelzer, E. H. Non-invasive long-term fluorescence live imaging of Tribolium castaneum embryos. Development, dev. 108795 (2014).

Szymczak-Workman, A. L., Vignali, K. M. \& Vignali, D. A. Design and construction of 2A peptide-linked multicistronic vectors. Cold Spring Harbor Protocols 2012, pdb. ip067876 (2012).

Tanaka, N. K., Tanimoto, H. \& Ito, K. Neuronal assemblies of the Drosophila mushroom body. Journal of Comparative Neurology 508, 711-755 (2008).

Technau, G. M., Berger, C. \& Urbach, R. Generation of cell diversity and segmental pattern in the embryonic central nervous system of Drosophila. Developmental dynamics: an official publication of the American Association of Anatomists 235, 861-869 (2006).

Therianos, S., Leuzinger, S., Hirth, F., Goodman, C. S. \& Reichert, H. Embryonic development of the Drosophila brain: formation of commissural and descending pathways. Development 121, 3849-3860 (1995).

Tosches, M. A. \& Arendt, D. The bilaterian forebrain: an evolutionary chimaera. Current opinion in neurobiology 23, 1080-1089 (2013).

Trauner, J., Schinko, J., Lorenzen, M. D., Shippy, T. D., Wimmer, E. A., Beeman, R. W., Klingler M., Bucher G. \& Brown, S. J. Large-scale insertional mutagenesis of a coleopteran stored grain pest, the red flour beetle Tribolium castaneum, identifies embryonic lethal mutations and enhancer traps. BMC biology 7, 73 (2009).

Truman, J. W. \& Bate, M. Spatial and temporal patterns of neurogenesis in the central nervous system of Drosophila melanogaster. Developmental biology 125, 145-157 (1988). 
Urbach, R. \& Technau, G. M. Early steps in building the insect brain: neuroblast formation and segmental patterning in the developing brain of different insect species. Arthropod structure \& development 32, 103-123 (2003a).

Urbach, R. \& Technau, G. M. Molecular markers for identified neuroblasts in the developing brain of Drosophila. Development 130, 3621-3637 (2003b).

Urbach, R. \& Technau, G. M. Neuroblast formation and patterning during early brain development in Drosophila. Bioessays 26, 739-751 (2004).

Vitzthum, H., Homberg, U. \& Agricola, H. Distribution of Dip-allatostatin I-like immunoreactivity in the brain of the locust Schistocerca gregaria with detailed analysis of immunostaining in the central complex. Journal of Comparative Neurology 369, 419-437 (1996).

Walldorf, U., Kiewe, A., Wickert, M., Ronshaugen, M. \& McGinnis, W. Homeobrain, a novel paired-like homeobox gene is expressed in the Drosophila brain. Mechanisms of development 96, 141-144 (2000).

Walsh, K. T. \& Doe, C. Q. Drosophila embryonic type II neuroblasts: origin, temporal patterning, and contribution to the adult central complex. Development, dev. 157826 (2017).

Wang, J., Zhang, H., Wang, H., Zhao, S., Zuo, Y., Yang, Y., \& Wu, Y. Functional validation of cadherin as a receptor of Bt toxin Cry1Ac in Helicoverpa armigera utilizing the CRISPR/Cas9 system. Insect biochemistry and molecular biology 76, 11-17 (2016).

Wegerhoff, R. \& Breidbach, O. Structure and development of the larval central complex in a holometabolous insect, the beetle Tenebrio molitor. Cell and tissue research 268, 341-358 (1992).

Wegerhoff, R., Breidbach, O. \& Lobemeier, M. Development of locustatachykinin immunopositive neurons in the central complex of the beetle Tenebrio molitor. Journal of Comparative Neurology 375, 157-166 (1996).

Weinrich, A., Kunst, M., Wirmer, A., Holstein, G. R. \& Heinrich, R. Suppression of grasshopper sound production by nitric oxide-releasing neurons of the central complex. Journal of comparative physiology A 194, 763-776 (2008).

Weng, M., Golden, K. L. \& Lee, C.-Y. dFezf/Earmuff maintains the restricted developmental potential of intermediate neural progenitors in Drosophila. Developmental cell 18, 126-135 (2010).

Weng, R. \& Cohen, S. M. Control of Drosophila Type I and Type II central brain neuroblast proliferation by bantam microRNA. Development 142, 3713-3720 (2015).

Wheeler, S. R., Carrico, M. L., Wilson, B. A., Brown, S. J. \& Skeath, J. B. The expression and function of the achaete-scute genes in Tribolium castaneum reveals conservation and variation in neural pattern formation and cell fate specification. Development 130, 
4373-4381 (2003).

Williams, J. Anatomical studies of the insect central nervous system: A ground-plan of the midbrain and an introduction to the central complex in the locust, Schistocerca gregaria (Orthoptera). Journal of Zoology 176, 67-86 (1975).

Williams, J. \& Boyan, G. Building the central complex of the grasshopper Schistocerca gregaria: axons pioneering the $w, x, y, z$ tracts project onto the primary commissural fascicle of the brain. Arthropod structure \& development 37, 129-140 (2008).

Williams, J., Guentner, M. \& Boyan, G. Building the central complex of the grasshopper Schistocerca gregaria: temporal topology organizes the neuroarchitecture of the $w, x, y, z$ tracts. Arthropod Structure \& Development 34, 97-110 (2005).

Xie, Y., Li, X., Deng, X., Hou, Y., O'Hara, K., Urso, A., Peng Y., Chen L. \& Zhu, S. The Ets protein Pointed prevents both premature differentiation and dedifferentiation of Drosophila intermediate neural progenitors. Development, dev. 137281 (2016).

Yaguchi, J., Angerer, L. M., Inaba, K. \& Yaguchi, S. Zinc finger homeobox is required for the differentiation of serotonergic neurons in the sea urchin embryo. Developmental biology $363,74-83$ (2012).

Yaguchi, S., Yaguchi, J., Angerer, R. C. \& Angerer, L. M. A Wnt-FoxQ2-nodal pathway links primary and secondary axis specification in sea urchin embryos. Developmental cell 14, 97-107 (2008).

Yaguchi, S., Yaguchi, J., Wei, Z., Shiba, K., Angerer, L. M., \& Inaba, K. ankAT-1 is a novel gene mediating the apical tuft formation in the sea urchin embryo. Developmental biology 348, 67-75 (2010).

Yang, J. S., Awasaki, T., Yu, H. H., He, Y., Ding, P., Kao, J. C., \& Lee, T. Diverse neuronal lineages make stereotyped contributions to the Drosophila locomotor control center, the central complex. Journal of Comparative Neurology 521, 2645-2662 (2013).

Young, J. \& Armstrong, J. Building the central complex in Drosophila: the generation and development of distinct neural subsets. Journal of Comparative Neurology 518, 1525-1541 (2010).

Younossi-Hartenstein, A., Nassif, C., Green, P. \& Hartenstein, V. Early neurogenesis of the Drosophila brain. Journal of Comparative Neurology 370, 313-329 (1996).

Younossi-Hartenstein, A., Nguyen, B., Shy, D. \& Hartenstein, V. Embryonic origin of the Drosophila brain neuropile. Journal of Comparative Neurology 497, 981-998 (2006).

Zacharias, D., Leslie, J., Williams, D., Meier, T. \& Reichert, H. Neurogenesis in the insect brain: cellular identification and molecular characterization of brain neuroblasts in the grasshopper embryo. Development 118, 941-955 (1993).

Zaffran, S., Das, G. \& Frasch, M. The NK-2 homeobox gene scarecrow (scro) is expressed in pharynx, ventral nerve cord and brain of Drosophila embryos. Mechanisms of

development 94, 237-241 (2000). 
Zaffran, S., Küchler, A., Lee, H.-H. \& Frasch, M. biniou (FoxF), a central component in a regulatory network controlling visceral mesoderm development and midgut morphogenesis in Drosophila. Genes \& development 15, 2900-2915 (2001).

Zhu, L., Mon, H., Xu, J., Lee, J. M. \& Kusakabe, T. CRISPR/Cas9-mediated knockout of factors in non-homologous end joining pathway enhances gene targeting in silkworm cells. Scientific reports 5, 18103 (2015).

Zhu, S., Barshow, S., Wildonger, J., Jan, L. Y. \& Jan, Y.-N. Ets transcription factor Pointed promotes the generation of intermediate neural progenitors in Drosophila larval brains. Proceedings of the National Academy of Sciences 108, 20615-20620 (2011). 


\section{Appendix}

\subsection{Abbreviations}

$2 A$

$\mathrm{AL}$

A-PD

A-PV

bhsp

CB

CNS

CRISPR

CX

DAPI

DC

Dm

DSB

DsRed

dsRNA

EB

EGFP

FAM

FB

GFP

GMC

gRNA

HDR self cleaving peptide allowing polycistronic expression

antennal lobe

anterior-posterodorsal

anterior-posteroventral

regulatory region of heat shock protein

central body

central nervous system

clustered regularly interspaced short palindromic repeats

central complex

4',6-diamidino-2-phenylindole

deuterocerebrum

prefix, Drosophila melanogaster

double stranded break

Discosoma sp. red fluorescent protein

double-stranded RNA

ellipsoid body

enhanced GFP

FoxQ2 anteromedian

fan-shaped body

green fluorescent protein

ganglion mother cell

guide RNA

homology directed repair 
His

hsp68

IPTG

L1

LAL

LSM

$\mathrm{MB}$

NB

NHJE

NO

PB

PC

$\mathrm{PI}$

pRNAi

RNAi

$S B$

SUMO

SV40

$T c$

TC

TSA

VNC

vw

wt hexahistidine

heat shock protein 68

Isopropyl $\beta$-D-1-thiogalactopyranoside

first larval instar

lateral accessory lobe

laser scanning microscope

mushroom body

neuroblast

non-homologous end joining

noduli

protocerebral bridge

protocererbrum

pars intercerebralis

parental RNAi

RNA interference

San Bernadino

small ubiquitin-like modifier

stop/poly adenylation-signal

prefix, Tribolium castaneum

tritocerebrum

tyramide signal amplification

ventral nerve cord

vermillion white

wild type 


\subsection{Primers and gRNAs used in this work}

Table 7.1 Primers sequences and purposes used for cloning.

\begin{tabular}{|c|c|c|}
\hline Primer & Sequence & Purpose \\
\hline BH_N_ter_fwd & $\begin{array}{l}\text { CCAGGTCTCATGGTATGTGCAGTAACGAGA } \\
\text { CTCCGG }\end{array}$ & \multirow{2}{*}{$\begin{array}{l}\text { Cloning of } T c-f o x Q 2 \\
\mathrm{~N} \text {-terminal fragment }\end{array}$} \\
\hline BH_N_ter_rev & $\begin{array}{l}\text { GGGGGTCTCCTCGAGGAAGAGCGGGTAG } \\
\text { CAG }\end{array}$ & \\
\hline BH_C_ter_fwd & $\begin{array}{l}\text { CCAGGTCTCATGGTTCCACGTCCGTTTATCA } \\
\text { CAC }\end{array}$ & \multirow{2}{*}{$\begin{array}{l}\text { Cloning of } T c-f o x Q 2 \\
\text { C-terminal fragment }\end{array}$} \\
\hline BH_C_ter_rev & $\begin{array}{l}\text { GGGGGTCTCCTCGAGTTAAGAGTCTGTGG } \\
\text { TGTCGGTGGC }\end{array}$ & \\
\hline BH_gRNA3_fwd & GCCACTGTTACCAAATATTGGTCC & \multirow{2}{*}{$\begin{array}{l}\text { gRNA testing by } \\
\text { T7 assay }\end{array}$} \\
\hline BH_gRNA3_rev & GCGGTTTGTACGCAAATTTG & \\
\hline RT1_EGFP_bhsp_rev & $\begin{array}{l}\text { TGCTCACCATGTTTGACTTTGAATTCACTA } \\
\text { GTAAATAATTCACTCAACTTTGTTAAAG }\end{array}$ & \multirow{4}{*}{$\begin{array}{l}\text { Cloning of bhsp, } \\
\text { EGFP, } 2 \mathrm{~A} \text { and Cre }\end{array}$} \\
\hline RT2_bhsp_EGFP_fwd & $\begin{array}{l}\text { AGTGAATTCAAAGTCAAACATGGTGAGCA } \\
\text { AGGGCG }\end{array}$ & \\
\hline RT3_2A_EGFP_rev & $\begin{array}{l}\text { GTCTCCTGCTTGCTTTAACAGAGAGAAGT } \\
\text { TCGTGGCTCCGGATCCCTTGTACAGCTCGT } \\
\text { CCATGCC }\end{array}$ & \\
\hline RT4_2A_Cre_fwd & $\begin{array}{l}\text { GCCACGAACTTCTCTCTGTTAAAGCAAGC } \\
\text { AGGAGACGTGGAAGAAAACCCCGGTCCT } \\
\text { ATGTCCAATTTACTGACCGTACACCAA }\end{array}$ & \\
\hline RT5_3xP3_fwd & $\begin{array}{l}\text { TCTAGACATTATTCATTAGAGACTAATTCA } \\
\text { ATTAGAGCTAATTCAATTAGGATCC }\end{array}$ & \multirow{5}{*}{$\begin{array}{l}\text { Cloning of } 3 \times \mathrm{P} 3 \text {, } \\
\text { vermillion and Sv40 }\end{array}$} \\
\hline RT6_Sv40_Cre_rev & $\begin{array}{l}\text { AATGGAAACAATTAAGATGAGTTTGGACA } \\
\text { AACCACA }\end{array}$ & \\
\hline RT7_Sv40_Cre_fwd & $\begin{array}{l}\text { TTGTCCAAACTCATCTTAATTGTTTCCATTC } \\
\text { GACACGT }\end{array}$ & \\
\hline RT8_Sv40_vw_rev & $\begin{array}{l}\text { GTATGGCTGATTATGACTAATCGCCATCTT } \\
\text { CCAGCA }\end{array}$ & \\
\hline RT9_Sv40_vw_fwd & $\begin{array}{l}\text { GGAAGATGGCGATTAGTCATAATCAGCCA } \\
\text { TACCACA }\end{array}$ & \\
\hline RT10_ebony_fwd & $\begin{array}{l}\text { GTCGGGCCCGAACCGGGCAGCCCGCCTCC } \\
\text { TGGCGTTTCATATATAAGCGCGGTCTCG }\end{array}$ & $\begin{array}{l}\text { Cloning of ebony } \\
\text { site with Apal }\end{array}$ \\
\hline RT11_yellow_fwd & $\begin{array}{l}\text { GTCTCTAGAGCGATATAGTTGGAGCCAGC } \\
\text { TGGATTATTCATTAGAGACTAATTC }\end{array}$ & $\begin{array}{l}\text { Cloning of yellow } \\
\text { site with } X b a l\end{array}$ \\
\hline
\end{tabular}




\begin{tabular}{|c|c|c|}
\hline BH_AG_fwd & GCGCTGGCATTTTTAAATCACG & \multirow{3}{*}{$\begin{array}{l}\text { Testing the } \\
\text { insertion site }\end{array}$} \\
\hline BH_AG_rev & ATACTGTAGAGCTGGAGCC & \\
\hline BH_EGFP_rev & TGGTGCAGATGAACTTCAG & \\
\hline BH_insertUp_fwd & GCGCTGGCATTTTTAAATCACG & \multirow{3}{*}{$\begin{array}{l}\text { Establishing the } \\
\text { homozygous stock }\end{array}$} \\
\hline $\mathrm{BH}$ insert_fwd & TGCTTTGAGTCGCTTGCTTA & \\
\hline BH_insertDown_rev & CCCTCAGAAACCCTCAGTTG & \\
\hline
\end{tabular}

Table 7.2 gRNAs target sequences and oligos used for generating gRNAs. The PAM sequence is marked in red. The orange sequence represents the complementary overhangs to the vector generated by Bsal digestion.

\begin{tabular}{|l|l|l|c|}
\hline $\begin{array}{c}\text { Name } \\
\text { of } \\
\text { gRNA }\end{array}$ & Genomic target sequence & Sense oligo & Antisense oligo \\
\hline gRNA1 & GGGCGTTACTGTCACCCTCCAGG & TTCGGGCGTTACTGTCACCCTCC & AAACGGAGGGTGACAGTAACGCC \\
\hline gRNA2 & GTGGCGGGGCGGAGCCAACGCGG & TTCGTGGCGGGGCGGAGCCAACG & AAACCGTTGGCTCCGCCCCGCCA \\
\hline gRNA3 & GTGAAGCACTGAATCCTACCTGG & TTCGTGAAGCACTGAATCCTACC & AAACGGTAGGATTCAGTGCTTCA \\
\hline gRNA4 & GAAGCACTGAATCCTACCTGGGG & TTCGAAGCACTGAATCCTACCTG & AAACCAGGTAGGATTCAGTGCTT \\
\hline gRNA5 & GAATCTGCAGACACAACCCAAGG & TTCGAATCTGCAGACACAACCCA & AAACTGGGTTGTGTCTGCAGATT \\
\hline $\begin{array}{l}\text { gRNA- } \\
\text { eb }\end{array}$ & GAACCGGGCAGCCCGCCTCCTGG & TTCGAACCGGGCAGCCCGCCTCC & AAACGGAGGCGGGCTGCCCGGTT \\
\hline $\begin{array}{l}\text { gRNA- } \\
\text { ye }\end{array}$ & GCGATATAGTTGGAGCCAGCTGG & TTCGCGATATAGTTGGAGCCAGC & AAACGCTGGCTCCAACTATATCG \\
\hline
\end{tabular}

Table 7.3 Plasmids used as PCR templates and molecular cloning.

\begin{tabular}{|l|l|}
\hline Plasmid name & Description \\
\hline pJET1.2 & backbone for knock-in construct cloning \\
\hline$\# 82$ pBac[3xP3-gTc'v; Tc'ems-overex] & used to amplify 3xP3, Tc-vw \\
\hline$\# 169$ pSLfa[Tc-hsp_p-ECFP-SV40] & used to amplify SV40 \\
\hline$\# 133$ pSLfa[Tc'hsp5'-Cre recomb-3'UTR]fa & used to amplify Cre \\
\hline$\# 65$ pBac[3xP3-gTc'v; Tc'hsp-Promotor RC] & used to amplify bhsp \\
\hline
\end{tabular}




\subsection{Quantification of the cell number in this study}

Table 7.4 Number of Tc-FoxQ2 ${ }^{+}$neuroblasts during embryogenesis.

\begin{tabular}{llllllll}
\hline Stage/No. & No.1 & No.2 & No.3 & No.4 & No.5 & No.6 & Mean \\
\hline NS8 & 15 & 16 & 15 & 12 & 17 & 16 & 15 \\
NS11 & 10 & 9 & 9 & 11 & 12 & 10 & 10 \\
NS14 & 6 & 5 & 6 & 5 & 7 & 5 & 6 \\
\hline
\end{tabular}

Table 7.5 Number of cells in respective groups at stage NS13 of Tc-foxQ2 enhancer trap line.

\begin{tabular}{lcccccc}
\hline Location/No. & No.1 & No.2 & No.3 & No.4 & No.5 & Mean \\
\hline $\begin{array}{l}\text { Anterior } \\
\text { neuroectoderm }\end{array}$ & 89 & 93 & 84 & 85 & 96 & 89 \\
\hline Median lineage & 26 & 28 & 33 & 31 & 24 & 28 \\
\hline
\end{tabular}

Table 7.6 Number of cells in respective groups at stage NS13 of Tc-foxQ2 enhancer trap line in Tc-foxQ2 RNAi.

\begin{tabular}{lllllll}
\hline Location/No. & No.1 & No.2 & No.3 & No.4 & No.5 & Mean \\
\hline $\begin{array}{l}\text { Anterior } \\
\text { neuroectoderm }\end{array}$ & 45 & 50 & 28 & 27 & 43 & 39 \\
\hline Median lineage & 11 & 10 & 9 & 6 & 8 & 9 \\
\hline
\end{tabular}

Table 7.7 Number of cells in the median lineage at stage NS14 of Tc-foxQ2 enhancer trap line.

\begin{tabular}{lllllll}
\hline Location/No. & No.1 & No.2 & No.3 & No.4 & No.5 & Mean \\
\hline Median lineage & 43 & 37 & 40 & 44 & 39 & 41 \\
\hline
\end{tabular}


Table 7.8 Number of cells in respective groups at stage NS15 of Tc-foxQ2 enhancer trap line.

\begin{tabular}{lccccc}
\hline Location/No. & No.1 & No.2 & No.3 & No.4 & Mean \\
\hline Median lineage & 68 & 62 & 71 & 58 & 65 \\
Lateral lineage & 11 & 18 & 15 & 17 & 15 \\
\hline
\end{tabular}

Table 7.9 Number of cells in median and lateral lineages at stage NS15 of Tc-foxQ2 enhancer trap line after Tc-foxQ2 RNAi.

\begin{tabular}{lccccc}
\hline Location/No. & No.1 & No.2 & No.3 & No.4 & Mean \\
\hline $\begin{array}{l}\text { Median lineage } \\
\text { and lateral lineage }\end{array}$ & 34 & 28 & 36 & 32 & 33 \\
\hline
\end{tabular}

Table 7.10 Number of cells in respective groups of the line E035004.

\begin{tabular}{llllll}
\hline Location/No. & No.1 & No.2 & No.3 & No.4 & Mean \\
\hline Median group & 30 & 28 & 27 & 24 & 27 \\
Lateral group & 36 & 31 & 27 & 34 & 32 \\
Posterior group & 38 & 40 & 41 & 36 & 39 \\
Total number & 104 & 99 & 95 & 94 & 98 \\
\hline
\end{tabular}

Table 7.11 Number of cells in respective groups of line E035004 in Tc-foxQ2 RNAi.

\begin{tabular}{llllll}
\hline Location/No. & No.1 & No.2 & No.3 & No.4 & Mean \\
\hline Median group & 8 & 17 & 15 & 18 & 15 \\
Lateral group & 7 & 16 & 22 & 17 & 16 \\
Posterior group & 22 & 28 & 17 & 16 & 20 \\
Total number & 37 & 61 & 54 & 51 & 51 \\
\hline
\end{tabular}


Table 7.12 Number of cells in respective groups of line E011A-01.

\begin{tabular}{lccccccc}
\hline Location/No. & No.1 & No.2 & No.3 & No.4 & No.5 & No.6 & Mean \\
\hline Median group & 48 & 42 & 49 & 40 & 40 & 39 & 43 \\
Lateral group & 28 & 35 & 31 & 30 & 37 & 43 & 34 \\
Total number & 76 & 77 & 80 & 70 & 77 & 82 & 77 \\
\hline
\end{tabular}

Table 7.13 Number of cells in respective groups of line E011A-01 in Tc-foxQ2 RNAi.

\begin{tabular}{llllllll}
\hline Location/No. & No.1 & No.2 & No.3 & No.4 & No.5 & No.6 & Mean \\
\hline Median group & 30 & 25 & 34 & 21 & 23 & 21 & 26 \\
Lateral group & 19 & 18 & 15 & 13 & 9 & 10 & 14 \\
Total number & 49 & 43 & 48 & 34 & 32 & 31 & 40 \\
\hline
\end{tabular}

Table 7.14 Number of cells of Tc-rx reporter line in wt and in Tc-foxQ2 RNAi.

\begin{tabular}{llllllll}
\hline & No.1 & No.2 & No.3 & No.4 & No.5 & No.6 & Mean \\
\hline WT & 35 & 42 & 43 & 36 & 41 & 44 & 40 \\
RNAi & 11 & 9 & 7 & 11 & 12 & 6 & 9 \\
\hline
\end{tabular}




\subsection{Vectors used in this study}

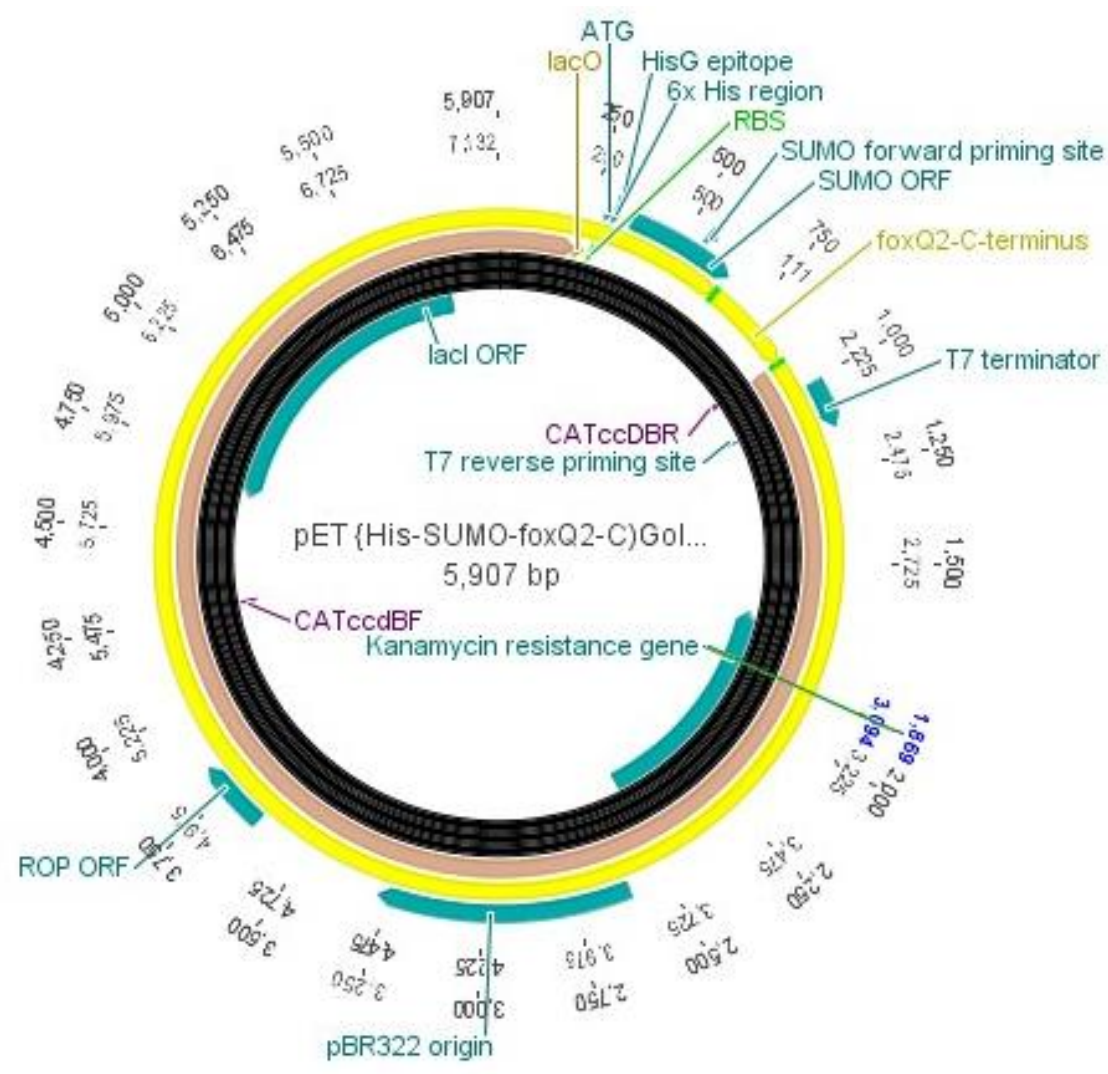

Figure 7.1 pET (His-SUMO-Tc'foxQ2-C). C terminal Tc-foxQ2 was cloned into pET SUMO vector which also contained His tag and SUMO. 


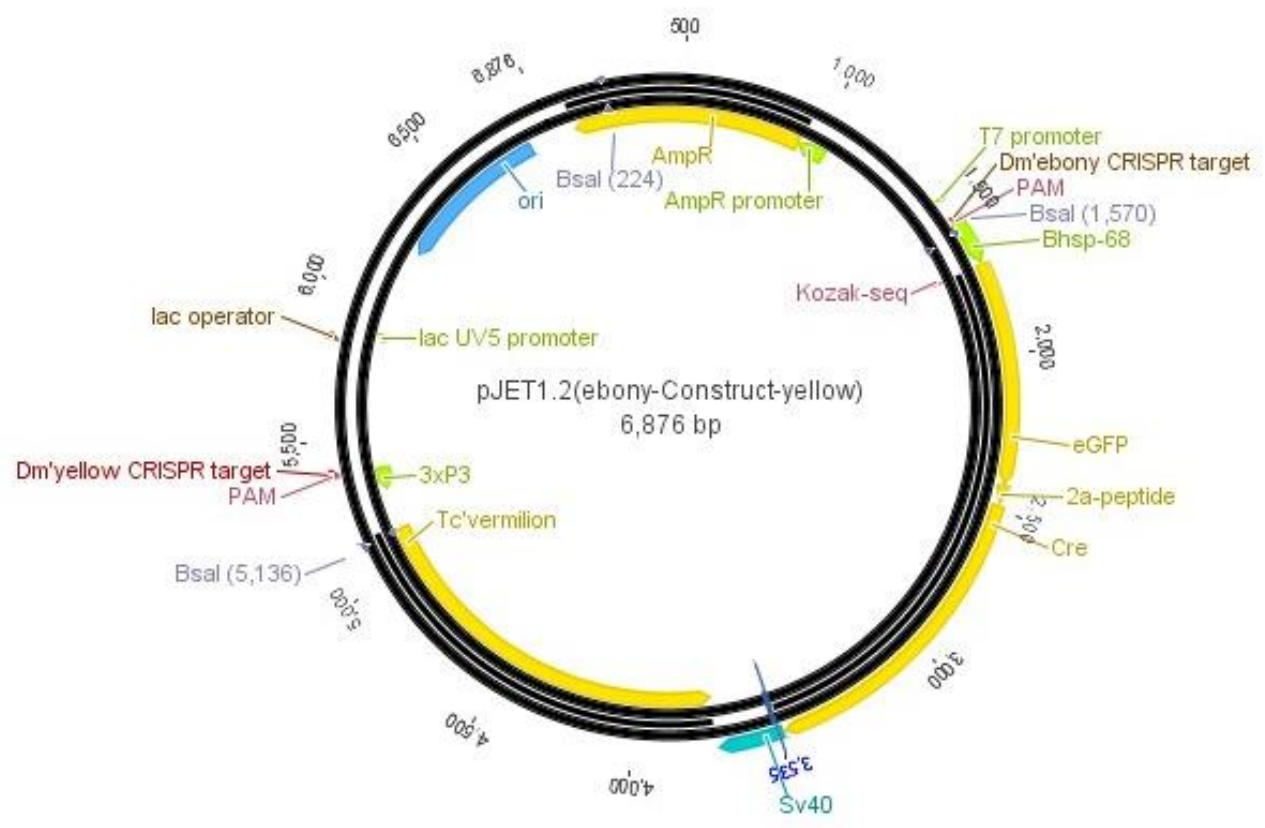

Figure 7.2 pJET1.2 (yellow-3xP3-Tc'vw-SV40; ebony-Tc'bhsp-EGFP-2A-Cre).

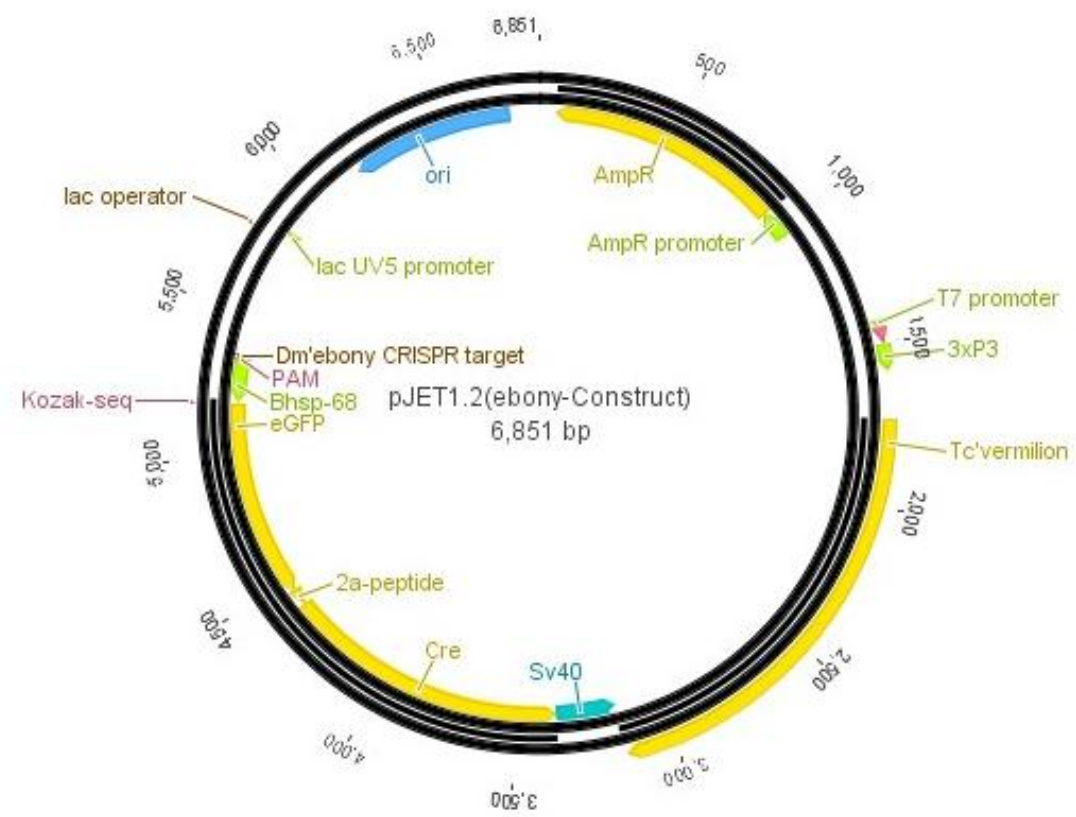

Figure 7.3 pJET1.2 (3xP3-Tc'vw-SV40; ebony-Tc'bhsp-EGFP-2A-Cre). 


\section{Curriculum Vitae}

\section{Personal profile}

$\begin{array}{ll}\text { Name: } & \text { Bicheng He } \\ \text { Nationality: } & \text { China } \\ \text { Date of Birth: } & \text { 16.07.1989 } \\ \text { Place of Birth: } & \text { Gansu, China }\end{array}$

\section{Address of current place of work}

$\begin{array}{ll}\text { University: } & \text { Georg-August-Universität Göttingen } \\ \text { Institute: } & \text { Johann-Friedrich-Blumenbach-Institute of Zoology and Anthropology } \\ \text { Department: } & \text { Department of Evolutionary Developmental Genetics } \\ \text { Street: } & \text { Justus-von-Liebig-Weg } 11 \\ \text { Town: } & 37077 \text { Göttingen } \\ \text { Country: } & \text { Germany } \\ \text { E-mail: } & \text { bicheng.he@stud.uni-goettingen.de }\end{array}$

\section{Education}

Since $10 / 2014$

Ph.D. Thesis "The role of $T c$-foxQ2 in central brain development in Tribolium castaneum" in the group of Prof. Dr. Gregor Bucher, Department of Developmental Evolutionary Genetics, Georg-August-Universität Göttingen.

09/2011-06/2014 Master Thesis "Fluorescent nanoparticle delivered dsRNA toward genetic control of insect pest" in the group of Prof. Dr. Jie Shen, Department of Entomology, China Agricultural University, Peking, China.

09/2007-06/2011 Bachelor study in the college of Plant Protection, Nanjing Agricultural University, Nanjing, China. 


\section{Publications}

Koniszewski, N. D. B., Kollmann, M., Bigham, M., Farnworth, M., He, B., Büscher, M., ... \& Bucher, G. (2016). The insect central complex as model for heterochronic brain development-background, concepts, and tools. Development genes and evolution, 226(3), 209-219.

Liu, X., He, B., Xu, Z., Yin, M., Yang, W., Zhang, H., ... \& Shen, J. (2015). A functionalized fluorescent dendrimer as a pesticide nanocarrier: application in pest control. Nanoscale, 7(2), 445-449.

Jiang, L., Ding, L., He, B., Shen, J., Xu, Z., Yin, M., \& Zhang, X. (2014). Systemic gene silencing in plants triggered by fluorescent nanoparticle-delivered double-stranded RNA. Nanoscale, 6(17), 9965-9969.

He, B., Chu, Y., Yin, M., Müllen, K., An, C., \& Shen, J. (2013). Fluorescent nanoparticle delivered dsRNA toward genetic control of insect pests. Advanced Materials, 25(33), 4580-4584. 